\title{
Superconducting Quantum Circuits
}





\section{Superconducting Quantum Circuits}

\section{Proefschrift}

ter verkrijging van de graad van doctor aan de Technische Universiteit Delft, op gezag van de Rector Magnificus prof.dr.ir. J.T. Fokkema, voorzitter van het College voor Promoties,

in het openbaar te verdedigen op vrijdag 13 december 2002 om 10.30 uur

door

\section{Johannes Balthasar Majer}

Diplomierter Physiker

Eidgenössische Technische Hochschule Zürich geboren te Weinfelden, Zwitserland. 
Dit proefschrift is goedgekeurd door de promotor:

Prof.dr.ir. J. E. Mooij

Samenstelling van de promotiecommissie:

Rector Magnificus, voorzitter

Prof.dr.ir. J. E. Mooij Technische Universiteit Delft, promotor

Dr. M. Grifoni, Technische Universiteit Delft, toegevoegd promotor

Prof.dr. T. P. Orlando, Massachusetts Institute of Technology, Verenigde Staten

Prof.dr. L. Levitov, Massachusetts Institute of Technology, Verenigde Staten

Prof.dr. P.H. Kes, Universiteit Leiden

Prof.dr. H. Rogalla, Universiteit Twente

Prof.dr.ir. P. Dewilde Technische Universiteit Delft

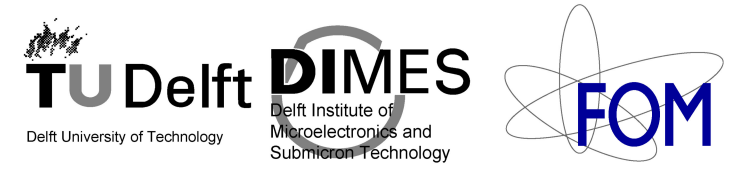

Published and distributed by: DUP Science

DUP Science is an imprint of

Delft University Press

P.O. Box $98 \quad$ Telephone: +31 152785678

2600 MG Delft Telefax: +31 152785706

The Netherlands $\quad$ E-mail: DUP@Library.TUDelft.NL

ISBN 90-407-2360-5

Keywords: superconductivity, Josephson effect, ratchet effect

Copyright (c) 2002 by Johannes B. Majer

All rights reserved. No part of the material protected by this copyright notice may be reproduced or utilized in any form or by any means, electronic or mechanical, including photocopying, recording or by any information storage and retrieval system, without permission from the publisher: Delft University Press.

Printed in the Netherlands 


\section{Preface}

This thesis is a result of four and a half years experimental research on Josephson quantum circuits. However, it is only a small fraction of all the work done in this time. Many things are hidden, like the work in the cleanroom, the restoration of the cryostat and many measurements without successful outcome. It was a very intensive and exciting time for me, certainly also because I explored a new country. Looking back I can hardly understand that I have now been in the Netherlands for more than four years already.

This thesis would not have been possible without the help of many people. First I would like to thank my supervisor Hans Mooij. I was always impressed with the new and original ideas that you came up with. When Milena Grifoni came to Delft, I found an excellent person to discuss our ratchet measurements. Milena, your enthusiasm is really contagious. Later also Joël Peguiron joined the ratchet team. For me it was always a pleasure to go upstairs and discuss the ratchets. I hope we can continue the collaboration for a long time. During my time as a Ph.D. I supervised four students during their 'afstuderen': Mark Tusveld, Jeremy Butcher, Floor Paauw and Floris Zwanenburg. Our cooperation was certainly one of my best experiences in these four and a half years. I also enjoyed being a part of THE qubit team and would like to thank the members Kees Haarmans Caspar van der Wal, Alexander ter Haar, Adrian Lupascu, Irinel Chiorescu,Ton Wallast and many undergraduate students. At the end of my time here in Delft, Yasunobu Nakamura came here for his sabbatical. Yasu, I was deeply impressed with the way you worked, and I have learned a lot from you. When I 
faced a problem concerning Josephson physics I always found a good discussion partner in Peter Hadley. Peter, your persistent way of asking forced me to think very thoroughly and made me understand the problems much better. I would like to thank my roommates Onno Mantel, Pieter Heij, Eugen Onac and Erwin Slot for the nice atmosphere in room number one. When I faced technical problems I could always count on support from Bram van der Enden, Mascha van Oossanen, Leo Lander, Wim Schot, Willem den Braver and Leo Dam. Bram, I was always jealous that your army stories were 'stoerder' than mine and with Mascha I shared the fascination for broad tires and huge exhaust pipes. A special thanks goes to Raymond Schouten, who supported me with his excellent electronic skills and self composed music. In the DIMES clean room I could always count on help of Emile van der Drift, Bert de Groot, Anja van Langen-Suurling, Bernard Rousseeuw, Arnold van Run, Marc Zuiddam, Arjan van Zuuk and Hans Romijn. Furthermore I would like to thank our management assistants Ria van Heeren-van der Kramer, especially for helping me to fight against stubborn bankers, and Yuki French-Nakagawa, sugoi desune. Among many colleagues and friends I would especially like thank Jeroen Elzerman, Ronald Hanson, Wilfred Wielewaal van der Wiel, Laurens Willems van Beveren, Leo Kouwenhoven, Yann-le Mac-Kervennic, Günther Lientschnig, Silvano De Franceschi (your homepage is really cool), Lieven Vandersypen, Alberto Stromboli Morpurgo, Hon Tin Man, Dionne Klein and Daniel Huertes Hernando, Nathan Kemeling, Ewout Eijkelenboom, Michael Go, Lukas van Gorkom, Serge Lemay, Cees Dekker and many more people from QT, MB and NF. Please forgive me if I forgot to mention you here. Outside Delft I had a lot of very interesting discussions with many people. Especially I would like to mention here discussions with Terry Orlando, Leonid Levitov and Christoph Bruder.

I would like to thank paranimfen Liesbeth Venema and Eliane Flück for supporting me on D-day. With Liesbeth I spent a very pleasant time as the famous Storklaan 19 team. A special thanks goes also to Chosee Gösele, who was my computer guru and with whom I spent many nice evenings talking about X, Palms or just drinking 'een biertje'. I would like to thank all the people that I left back in Switzerland: Roger-Hotline-Lüchinger, Christian Hilbes, Dani Hug, Guido Burkard, Piitsch Messer, Susi, Wisi and Janine 
(d'Habühlers) and many more. Last but certainly not least to thank my parents and my sister for the support and encouragement during my time in the Netherlands.

Johannes B. Majer

Delft, November 2002 


\section{Contents}

1 Introduction $\mathbf{5}$

1.1 Vortices in Josephson junction arrays . . . . . . . . . . . 6

1.2 Josephson quantum circuits . . . . . . . . . . . . . 10

1.3 Fabrication method . . . . . . . . . . . . . . 12

1.4 Thesis layout . . . . . . . . . . . . . . . . 14

References . . . . . . . . . . . . . . . 15

2 Quantum ratchet effect for vortices $\quad 17$

2.1 Introduction . . . . . . . . . . . . . . . . . . . . 18

2.2 Quantum transport in a few band system . . . . . . . . . 18

2.3 Sample design . . . . . . . . . . . . . . . . . . . . . . . . . . . 24

2.4 Measurements . . . . . . . . . . . . . . . 27

2.5 Conclusions . . . . . . . . . . . . . . . . 36

References .................. 37

\section{Vortex transport}

in quasi one-dimensional

Josephson junction arrays $\quad 39$

3.1 Introduction . . . . . . . . . . . . . . . . . . 40

3.2 Vortex density of quasi one-dimensional Josephson junction

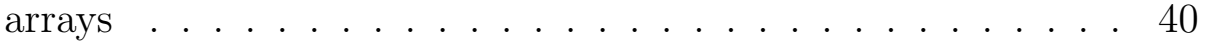

3.2.1 Theory .................... . . . . . 41

3.2.2 Sample design and measurements . . . . . . . . . . 43

3.2.3 Conclusion ... . . . . . . . . . . . . . . 44

3.3 Quantum vortices with weak interaction . . . . . . . . . 46

3.3.1 Sample fabrication and measurements . . . . . . . . 46 
3.3.2 Conclusions . . . . . . . . . . . . . . . . . . . . . . 49

References . . . . . . . . . . . . . . . . 52

4 Simple phase bias for superconducting circuits 53

4.1 Introduction . . . . . . . . . . . . . . . . 54

4.2 The phase bias ring . . . . . . . . . . . . . . . . 54

4.3 The $\pi$-SQUID and $\pi / 2$-SQUID $\ldots \ldots \ldots \ldots$

4.4 Applications . . . . . . . . . . . . . . . . 63

References ..................... . . 70

5 Coupling of qubits $\quad 73$

5.1 Introduction . . . . . . . . . . . . . . . . . 74

5.2 Persistent-current qubit as quasi-spin $\ldots \ldots \ldots . . \ldots 75$

5.3 Qubit coupling mechanisms . . . . . . . . . . . . 77

5.3 .1 Inductive coupling . . . . . . . . . . . . . 77

5.3 .2 Capacitive coupling . . . . . . . . . . . . . . . . . . . . 84

5.4 Sample layout and design . . . . . . . . . . . . . . . 86

5.5 Measurements . . . . . . . . . . . . . . . . . 90 90

5.6 Conclusions . . . . . . . . . . . . . . . . . . . . . 94

References . . . . . . . . . . . . . . . . 95

$\begin{array}{ll}\text { Summary } & 99\end{array}$

$\begin{array}{ll}\text { Samenvatting } & 103\end{array}$

$\begin{array}{ll}\text { Zusammenfassung } & 107\end{array}$

$\begin{array}{ll}\text { List of publications } & 111\end{array}$

$\begin{array}{ll}\text { Curriculum Vitae } & 113\end{array}$ 


\section{Chapter 1}

\section{Introduction}

Circuits based on Josephson junctions are outstanding systems to study quantum mechanical effects. With modern lithographic techniques junctions can be fabricated with well-defined properties. At low temperatures the dissipative effects freeze out and the junction behaves very underdamped.

The electrical properties of the junctions can be described by two competing energies $[1,2]$ : The Josephson coupling energy $E_{J}$ and the charging energy $E_{c}$. The Josephson coupling energy is a measure of the strength for the phase-coupling between the two superconductors. It is determined by the critical current $I_{0}$ of the junction according to $E_{J}=I_{0} \Phi_{0} / 2 \pi$. Here $\Phi_{0}=h / 2 e$ is the superconducting flux quantum. The supercurrent through the junction is given by the Josephson relation [3]: $I=I_{0} \sin (\gamma)$, where $\gamma$ is the superconducting phase difference across the junction. Due to the parallel plate geometry of the junction a capacitance $C$ is formed. The charging energy $E_{c}$ is the energy needed to move a single charge $e$ across the junction: $E_{c}=e^{2} / 2 C$.

Phenomenologically one describes all dissipative effects by a constant resistor $R[1,2]$. This leads to the resistively, capacitively shunted junction (RCSJ) model, which describes the dynamics with three channels (Fig. 1.1):

$$
I_{\mathrm{bias}}=\frac{V}{R}+C \frac{\partial V}{\partial t}+I_{0} \sin (\gamma)
$$

Here $V$ is the voltage across the junction which depends on the phase differ- 

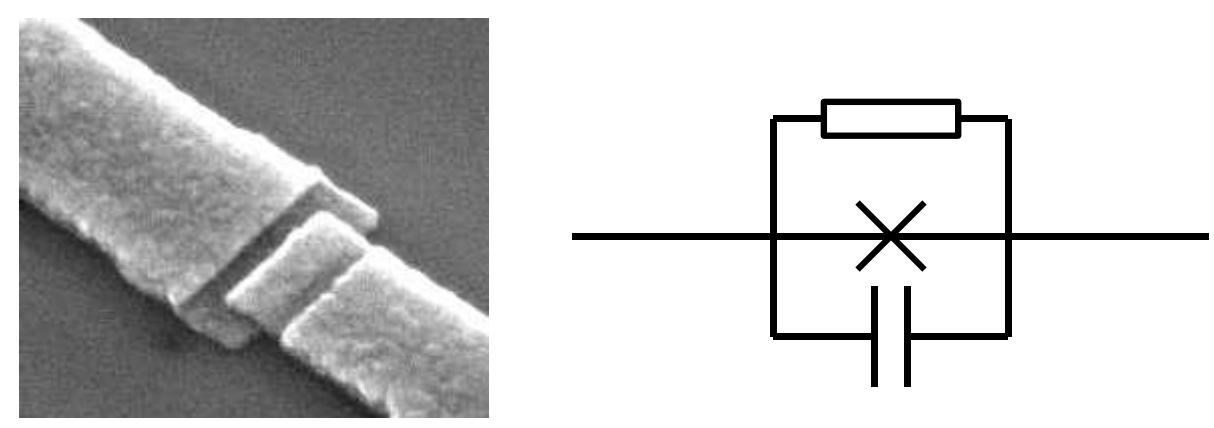

Figure 1.1: A Josephson junction consists of two superconductors which are coupled by a very thin insulating layer. Left: A scanning electron microscope image of a Josephson junction fabricated with shadow evaporation technique. Right: Resistively, capacitively shunted junction (RCSJ) model for a Josephson junction.

ence by the second Josephson equation

$$
V=\frac{\Phi_{0}}{2 \pi} \frac{\partial \gamma}{\partial t}
$$

If the junctions are placed in a circuit with superconducting loops, the phase differences $\gamma_{i}$ have to obey the fluxoid quantization condition:

$$
\sum_{\text {loop }} \gamma_{i}=2 \pi f+2 \pi n
$$

where $f=\Phi / \Phi_{0}$ with $\Phi$ the flux penetrating the loop and $n$ the number of fluxoids.

\subsection{Vortices in Josephson junction arrays}

A Josephson junction array consists of a regular arrangement of superconducting islands that are weakly coupled to each other by a Josephson junction. Using modern modern lithographic techniques a wide variety of different arrays can be fabricated. 
The arrays studied in this thesis are quasi one-dimensional. They consist of a long, narrow network of Josephson junctions arranged in a rectangular lattice (see Fig. 1.2). Typically the width $\mathrm{W}$ ranges from 5 to 7 cells and the length L from 300 to 1000 cells.

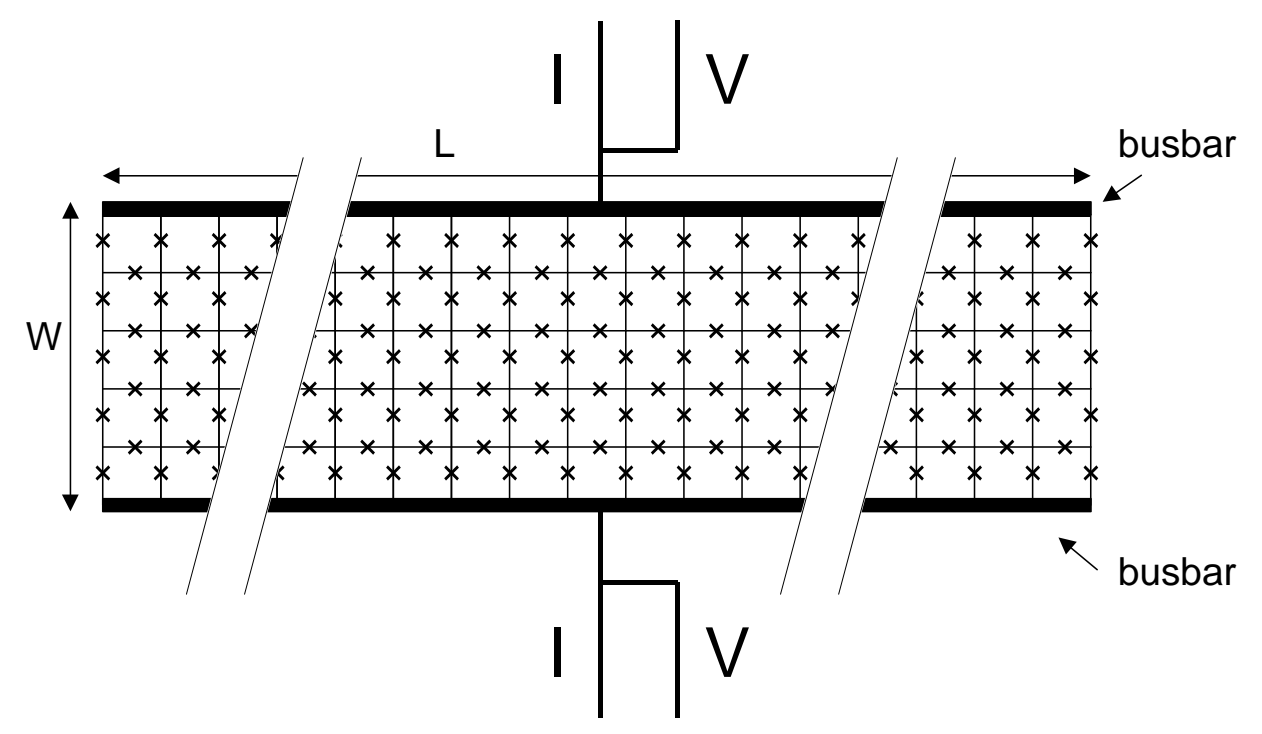

Figure 1.2: Schematic lay out of a Josephson junction array for one-dimensional vortex motion. Josephson junctions are represented by a cross, cells are areas enclosed by four junctions. Vortices are induced by an applied magnetic field perpendicular to the array. They are repelled by the busbars and forced to the middle row. Current is applied vertically in the figure between the busbars, homogeneously along the length. The current induces a potential gradient along the length. The motion of vortices through the array creates a voltage across the array.

Applying a magnetic field perpendicular to the array induces vortices in the system. Vortices are phase configurations, where the phase winds up by $2 \pi$ when going round a cell. The numbers of vortices in a cell is given by

$$
N=\left.\frac{1}{2 \pi} \sum_{\text {around cell }} \gamma_{i}\right|_{\gamma_{i} \in[-\pi \ldots \pi]}
$$

The expression $\gamma_{i} \in[-\pi \ldots \pi]$ means that the phase difference is rounded by multiples of $2 \pi$ such that it is in the interval $[-\pi \ldots \pi]$. Figures 1.3 and 1.4 
show lowest energy configurations for a frustration of $f=1 /(5 \cdot 29)$, resp. $f=6 /(5 \cdot 29)$. The phases $\varphi_{i}$ of the islands are related to the phase difference by

$$
\gamma=\varphi_{i}-\varphi_{j}-A_{i j} \quad \text { where } \quad A_{i j}=\frac{2 \pi}{\Phi_{0}} \int_{i}^{j} \mathbf{A} d \mathbf{l}
$$

Here $\mathbf{A}$ is the vector gauge, which is chosen to be $\mathbf{A}=2 \pi f(x / a) \mathbf{e}_{y}$, where $x$ is the position in $\mathrm{x}$-direction, $a$ the lattice constant and $\mathbf{e}_{y}$ the unit vector in $\mathrm{y}$-direction.
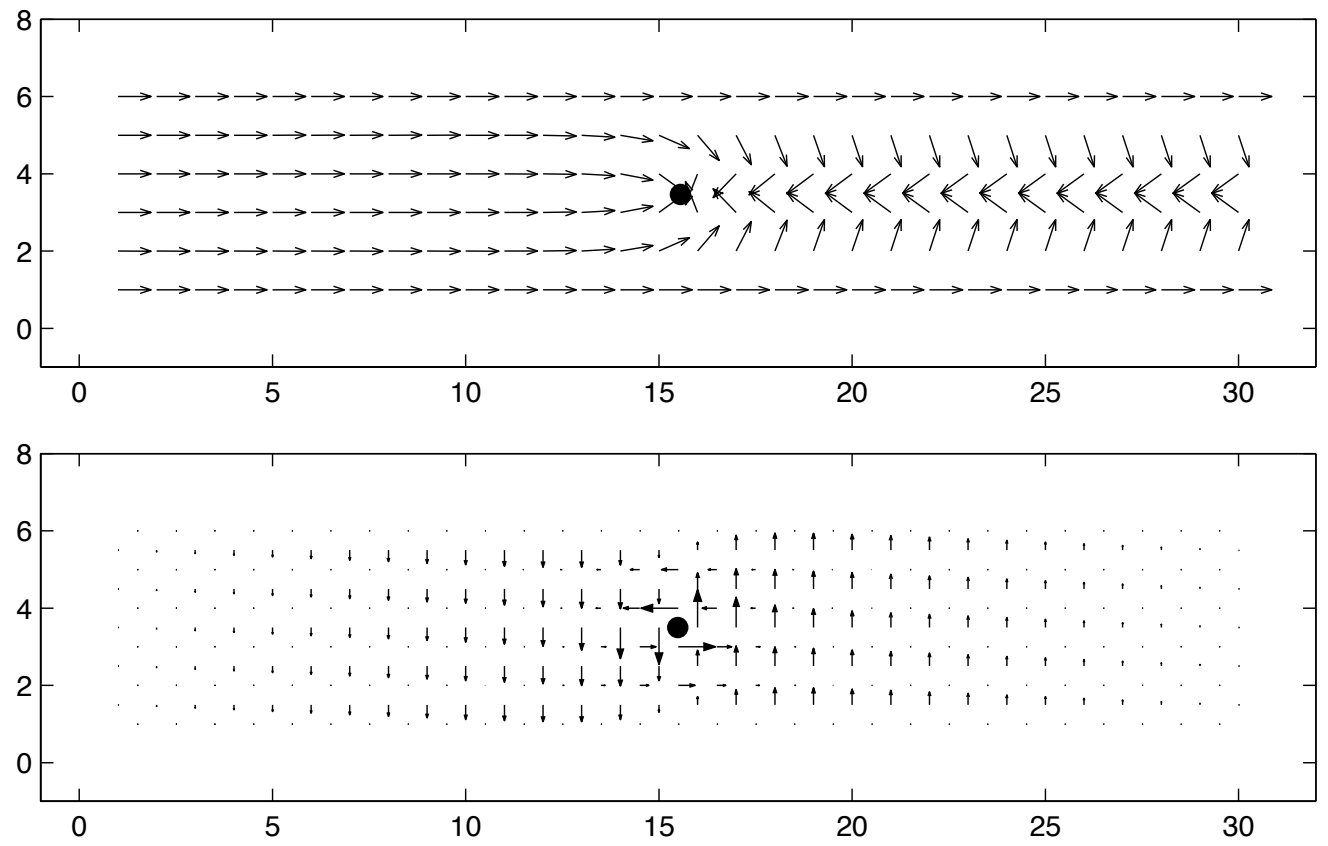

Figure 1.3: Phase and current configuration for an array as shown in figure 1.2 with one vortex in the array. Here one flux quantum is applied to the array, $f=1 /(5 \cdot 29)$. The dot indicates the position of the vortex (Eq. 1.4 ). Above: The direction of the arrows indicates the phases of the islands. Below: The length of the arrows indicates the current through the junctions.

The number of vortices in the array is equal to the number of flux quanta $\left(\Phi_{\text {tot }} / \Phi_{0}\right)$ applied to the entire array [4]. Therefore the one-dimensional vortex density $n_{V}$ (or equivalently the number of vortices per column) is equal 

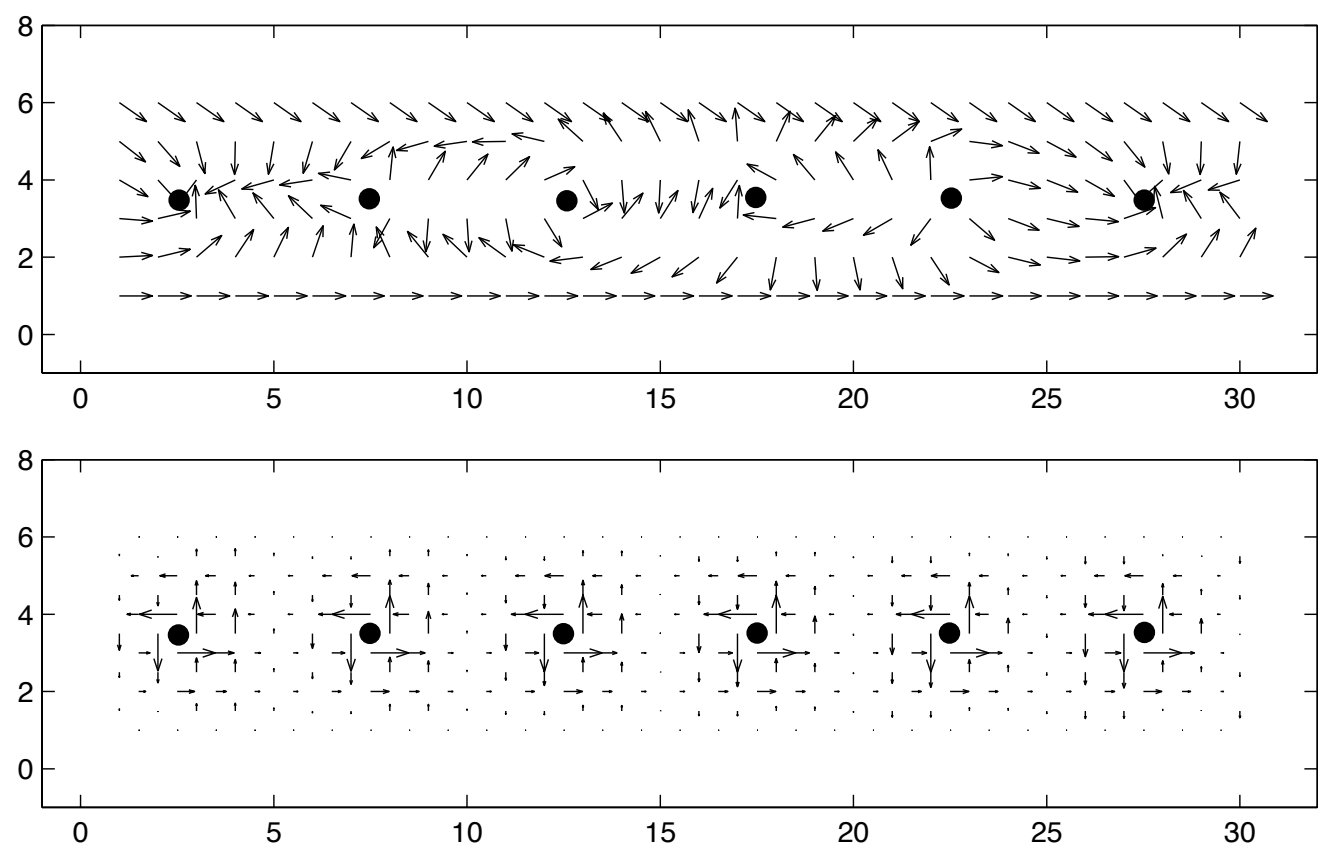

Figure 1.4: Phase and current configuration for an array as shown in figure 1.2 with six vortices in the array. Here six flux quanta are applied to the array, $f=6 /(5 \cdot 29)$. The dot indicates the position of the vortex (Eq. 1.4). Above: The direction of the arrows indicates the phases of the islands. Below: The length of the arrows indicates the current through the junctions.

to

$$
n_{V}=\frac{\Phi_{\text {tot }}}{\Phi_{0}} \frac{a}{L}
$$

The superconducting current and voltage electrodes along the length of the array (called busbars) repel the vortices, which consequently are forced to move along the centre row. These vortices behave like massive particles [5]. The mass of the vortex is proportional to the capacitance $C$ of the junctions, $m_{\mathrm{V}} \approx \Phi_{0}^{2} C / 2 a^{2}$. By injecting a bias current into the busbars a force can be exerted on the vortex. The vortex current (number of vortices passing per unit time) can be determined by measuring the voltage between the busbars.

One can calculate the classical dynamics of the whole array by solving Kirchhoff's law for each island and using equation (1.1) and (1.2) to describe 
the junction. In reference [6] one can find the dynamical solution for an array with a length of 29 cells and a width of 5 cells. Figure 1.5 shows the resulting voltage-current curves for different magnetic fields. One observes a critical current (the vortices are pinned to the array and cannot move) and then a linear increase of the voltage. With increasing magnetic field the voltage increases. This is due to the fact that the number of vortices increases. But one also observes that the critical current is decreased with increasing field, which is due to the decrease of the boundary potential [4].

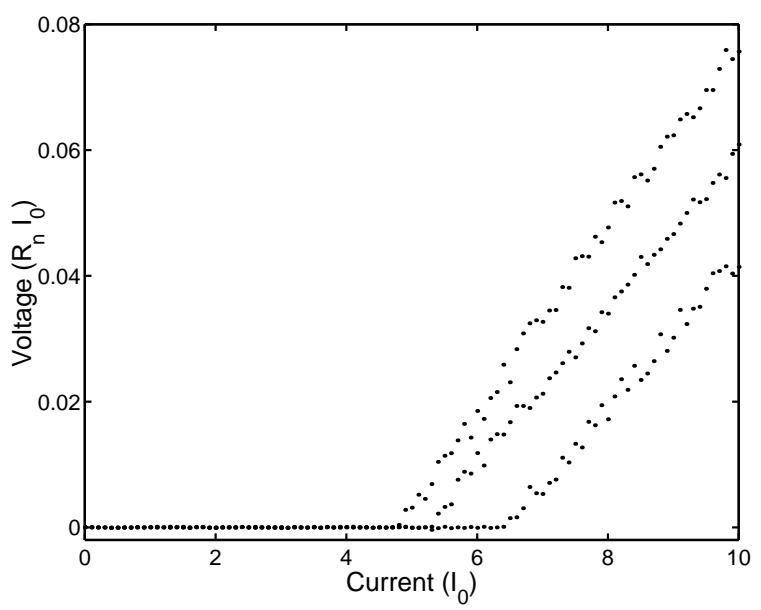

Figure 1.5: Voltage versus current curves obtained by solving the classical equations of the Josephson junction array. The different curves are for different magnetic fields applied: $f=4 /(5 \cdot 29), 5 /(5 \cdot 29)$ and $6 /(5 \cdot 29)$. The scattering is due to the random initial conditions.

\subsection{Josephson quantum circuits}

Up to now the vortex was considered as a classical particle. This is only valid in the limit of large $E_{J} / E_{c}$, which means that the vortex has a high mass and feels a high potential. However, when the ratio $E_{J} / E_{c}$ is decreased the potential gets weaker and the mass lower. The vortex shows quantum mechanical properties. This is due to the fact that phase and charge are conjugate variables. Instead of using equation 1.1 one has write down a 
Hamiltonian and diagonalize it. For a vortex moving in a periodic potential Bloch's equations have to be solved. The result, for a potential which is calculated in chapter 2 , is shown in figure 1.6. One observes energy bands well separated by an energy gap. Experimentally quantum properties for vortices have been demonstrated (For a review see [7]).

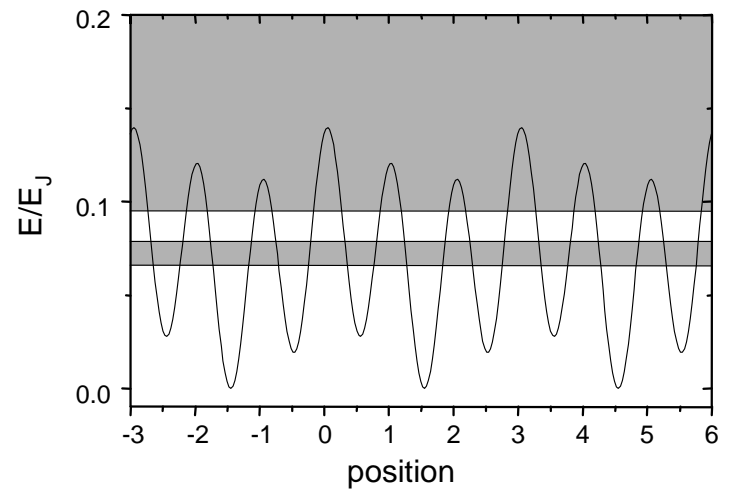

Figure 1.6: Vortex potential and energy bands for an array with $E_{J} / E_{c}=10$.

Solving the Hamiltonian for an array as shown in figure 1.2 is impossible. However, for a persistent-current qubit [8] with only three Josephson junctions this can be done numerically. 


\subsection{Fabrication method}

In this section we give an overview of the fabrication procedure which was used to make the small Josephson junctions. The method is called shadow evaporation technique and was invented by Dolan [10].

1. electron beam writing

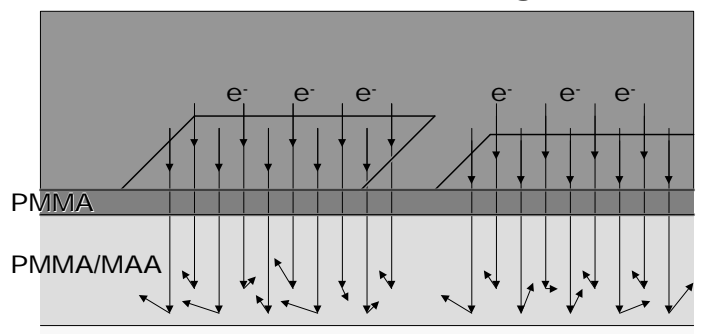

Substrate

\section{3. first aluminum evaporation}

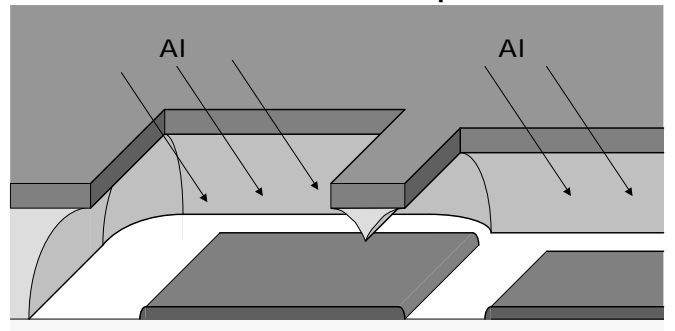

5. second aluminum evaporation

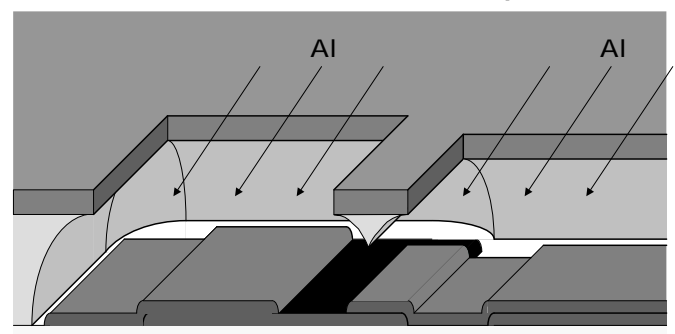

\section{2. development}

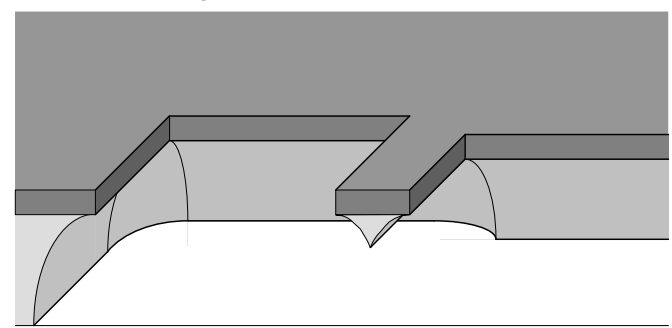

\section{4. oxidation}

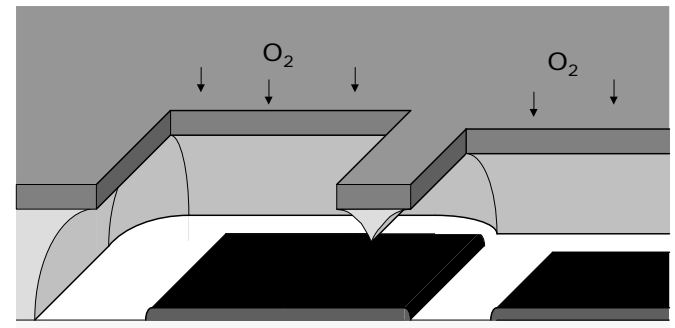

6. lift-off

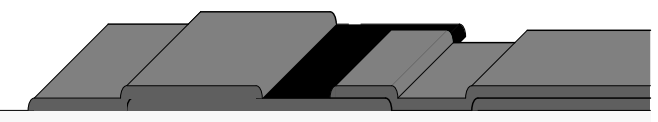

Figure 1.7: Schematic overview of the fabrication procedure.

The samples are fabricated on a silicon substrate with an insulating $\mathrm{SiO}_{2}$ layer on top. After cleaning the substrate with acids two resist layers are spun 
onto the substrate. The lower layer is a PMMA/MAA copolymer solved in Ethyl-Lactate and the upper layer is a solution of PMMA in chlorobenzene. Then the pattern of the sample is written with a high resolution electron beam lithography machine (Figure 1.7.1). After exposure, the resist is developed in 1:3 mixture of MIBK and iso-propanol for one minute (Figure 1.7.2). Due to the back-scattered electrons and the difference in materials, a larger area of the lower resist layer than of the upper resist layer is removed during development. This lead to an undercut and at certain places a free hanging bridge which is needed for the shadow evaporation. After defining the mask, the sample is mounted in an evaporator with a sample holder that can be rotated. First a $30 \mathrm{~nm}$ thin layer of aluminum is evaporated under an angle $\alpha$ (Figure 1.7.3). Then the thin insulating aluminum oxide is formed by exposing it with pure oxygen under a controlled pressure (Figure 1.7.4). In a second evaporation step a $50 \mathrm{~nm}$ aluminum layer is evaporated under an angle $-\alpha$ (Figure 1.7.5). The remaining resist layers and the aluminum on top of the resist are removed with acetone (Figure 1.7.6). Now the two aluminum layers overlap with each other at a region with very small area and with a very thin layer of insulating $\mathrm{Al}_{2} \mathrm{O}_{3}$ between. Figure 1.8 shows two scanning electron microscope images of a Josephon junction fabricated with this method. The two aluminum shadow layers are clearly visible.

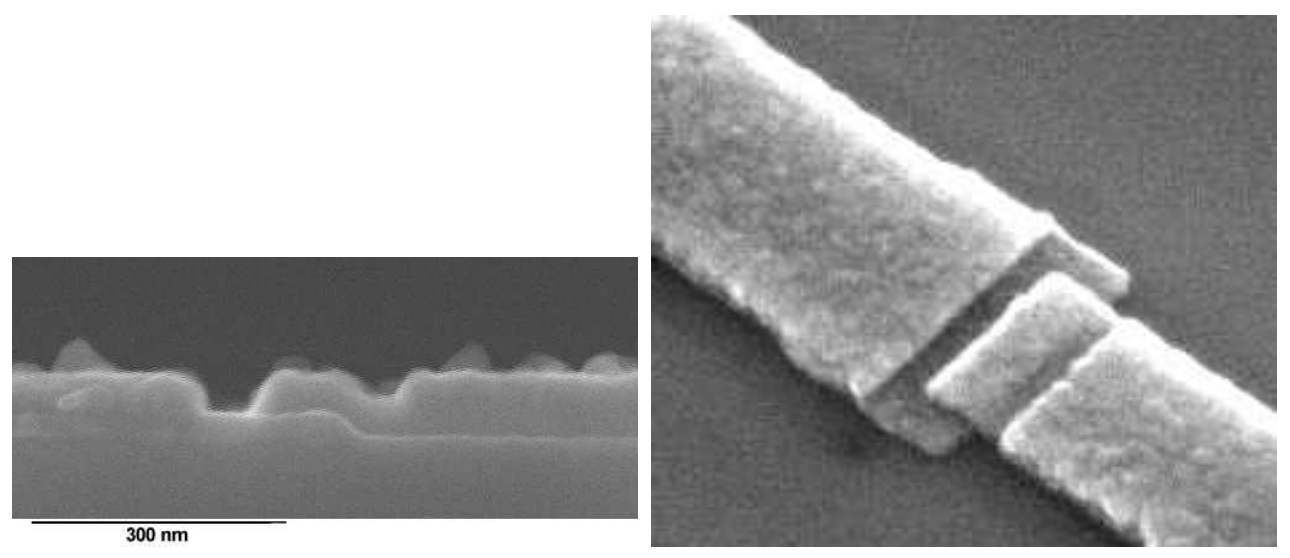

Figure 1.8: Scanning electron microscope images of a Josephon junction made by shadow evaporation technique. 


\subsection{Thesis layout}

The first two chapters contain experiments with quasi one-dimensional Josephson junction arrays. Chapter 2 describes an experiment on quantum ratchets. Here the quantum nature of the vortices is demonstrated by the outcome of the measurement. Chapter 4 presents a method to apply a fixed phase-bias, which can be applied in many superconducting circuits. The last chapter describes the coupling of two qubits. 


\section{References}

[1] M. Tinkham, "Introduction to Superconductivity," McGraw-Hill, New York, (1996).

[2] T. P. Orlando, K. A. Delin, "Foundations of Applied Superconductivity," Addison-Wesley Publishing Company (1991).

[3] B. D. Josephson, Phys. Lett. 1, 251 (1962).

[4] C. Bruder, L. I. Glazman, A. I. Larkin, J. E. Mooij, A. van Oudenaarden, "Phase transition in a chain of quantum vortices," Phys. Rev. B 59, 1383 (1999).

[5] T. P. Orlando, J. E. Mooij, H. S. J. van der Zant, "Phenomenological model of vortex dynamics in arrays of Josephson junctions," Phys. Rev. B 43, 10218

[6] http://qt.tn.tudelft.nl/ majer/thesis/vortex.html

[7] R. Fazio and H. van der Zant, "Quantum phase transitions and vortex dynamics in superconducting networks," Physics Reports, 355, 235 (2001).

[8] J. E. Mooij, T. P. Orlando, L. Tian. C. H. van der Wal, L. S. Levitov, and J. J. Mazo, "A Superconducting Persistent Current Qubit," Science 285, 1036 (1999).

[9] http://qt.tn.tudelft.nl/ ${ }^{\sim m a j e r / q u b i t ~(1991) . ~}$

[10] G. J. Dolan, "Offset masks for lift-off photoprocessing," Appl. Phys. Lett. 31, 337 (1977) 


\title{
Chapter 2
}

\section{Quantum ratchet effect for vortices}

\author{
J. B. Majer, M. Tusveld, J. E. Mooij \\ J. Peguiron, M. Ferreira, M. Grifoni
}

We have measured a quantum ratchet effect for vortices in a fabricated one-dimensional Josephson junction array. In this solid-state device the shape of the vortex potential energy, and consequently the band structure, can be accurately designed. This band structure determines the presence or absence of the ratchet effect, as observed in the presented experiments. A

theory for quantum ratchets with only few bands is developed, which qualitatively explains the experimental results. The quantum nature of transport is also revealed in a characteristic power-law dependence of the measured voltage-current characteristics. The ratchet effect increases with increasing dissipation.

The theoretical part of this chapter has been published in Physical Review Letters 89, 146801 and the experimental part has been submitted to Physical Review Letters. 


\section{$2.1 \quad$ Introduction}

A ratchet, i.e. an asymmetric periodic structure, yields the possibility to extract net particle flow from noise and unbiased driving [18, 2]. The physical principle of ratchets explains the working of molecular motors $[3,4,5]$. Quantum ratchets [6] can be used to guide electrons, atoms, or other quantum particles such as vortices in superconducting systems, in pre-assigned directions. Furthermore it allows the investigation of the influence of dissipation in quantum transport. Rectification of quantum fluctuations has so far been observed only in triangularly-shaped semiconductor heterostructures [7]. Here we report on the transport of quantum particles through a periodic potential with asymmetric unit cells, in particular on the quantum ratchet effect of vortices in quasi-one-dimensional Josephson junction arrays. First we develop a theory which describes the vortex transport in a system with only few energy bands. Then we show how a quantum ratchet potential can be realized with Josephson junction arrays. We end up with measurement results, which are compared to the theory.

\subsection{Quantum transport in a few band system}

In this section, we develop a theory for the motion of vortices in a quasi-onedimensional junction array. The theory assumes a single particle model. The goal of the theory is to calculate the speed $v$ of a vortex. Given the speed and the density of vortices per length $n$, the measurable voltage $V$ can be calculated

$$
V=n v \frac{\Phi_{0}}{a}
$$

Here $\Phi_{0}=h / 2 e$ is the superconducting flux quantum. The single particle assumption is justified by the experimental finding that the voltage scales with vortex density for small densities.

The model assumes a vortex which is moving in one-dimensional potential. Additionally the vortex feels an external driving force $F$ and friction. We consider the following Hamiltonian

$$
H=H_{\text {Ratchet }}+H_{\text {Bath }}+H_{\text {ext }}
$$


where $H_{\text {Ratchet }}=p^{2} / 2 m+V_{\text {Ratchet }}(x)$ is the Hamiltonian for a vortex with mass $m$ moving in the asymmetric periodic ratchet potential $V_{\text {Ratchet }}(x+L)=$ $V_{\text {Ratchet }}(x) . L$ is the period of the potential and therefore the length of one unit cell. The ratchet potential is optained by considering the static energy of the Josephson junction array [8]. Due to the periodicity of the potential, the solution of Schrödinger's equation yields Bloch bands $H_{\text {Ratchet }}\left|\Psi_{m, k}\right\rangle=$ $\varepsilon_{m}(k)\left|\Psi_{m, k}\right\rangle$, where $m$ denotes the band index and $k$ the wave-vector. Due to time reversal symmetry and periodicity the Bloch band energy has the following form

$$
\varepsilon_{m}(k)=E_{m}+\sum_{o=1}^{\infty} \frac{\Delta_{m}^{(o)}}{2} \cos (o k L)
$$

Here $E_{n}$ is the center of the energy band, while the $\Delta_{m}^{(o)}$ define the shape of the band, including the width of the energy band. The action of the external force is captured by $H_{\text {ext }}=x F$. Friction is modeled by weakly coupling the motion of the particle to a bath of harmonic oscillators [9].

$$
H_{\mathrm{Bath}}=\sum_{i=1}^{N} \frac{p_{i}^{2}}{2 m_{i}}+\frac{m_{i}}{2} \omega_{i}^{2}\left(x_{i}-c_{i} \frac{x}{m_{i} \omega_{i}^{2}}\right)^{2}
$$

Here $m_{i}$ is the mass and $\omega_{i}$ the resonance frequency of the $i^{\text {th }}$ harmonic oscillator in the bath. $c_{i}$ gives the strength of the coupling to the vortex. If the coupling is weak, the character of the bath is described by the spectral function $J(\omega)$. We assume a Ohmic form with a Drude cut-off

$$
J(\omega)=\frac{\eta \omega}{1+\left(\omega / \omega_{D}\right)^{2}}
$$

where $\eta$ is the friction coefficient and $\omega_{D}$ the cut-off frequency.

We introduce the Wannier states [17]

$$
|j, m\rangle=\frac{1}{\sqrt{N}} \sum_{k} e^{-i k j L}\left|\Psi_{m, k}\right\rangle
$$

which are localized at cell number $j$. The asymmetry of the potential is contained by the Wannier states and is reflected by relation $\langle x \mid j, m\rangle \neq$ $\langle-x \mid-j, m\rangle$. 
We restrict the Hamiltonian to nearest-neighbor coupling which leads to a tight binding Hamiltonian with $\varepsilon_{m}(k)=E_{m}+\left(\Delta_{m} / 2\right) \cos (k L)$, with $\Delta_{m}=\Delta_{m}^{(1)}$ Furthermore, we take only the first $M$ energy bands into account. Then the ratchet Hamiltonian has the following form

$$
\begin{aligned}
H_{\text {Ratchet }} & =\sum_{j=-\infty}^{\infty} \sum_{m=1}^{M} E_{m}|j, m\rangle\langle j, m| \\
& +\sum_{j=-\infty}^{\infty} \sum_{m=1}^{M} \frac{\Delta_{m}}{4}(|j, m\rangle\langle j+1, m|+| j+1, m\rangle\langle j, m|)
\end{aligned}
$$

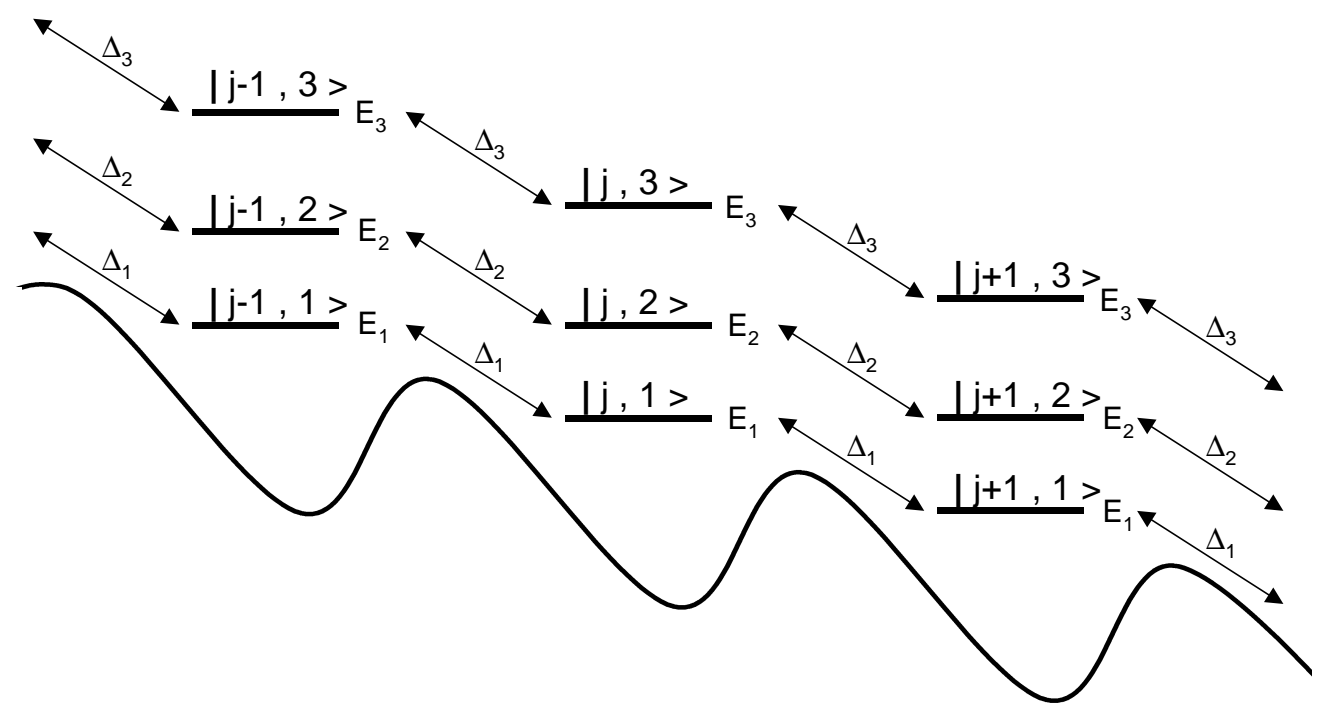

Figure 2.1: Graphical representation of the ratchet Hamiltonian Eq. (2.7) in the Wannier basis. The Wannier states $|j, m\rangle$ with energy $E_{m}$ are localized at the cell $j$ and coupled to their neighbors $|j \pm 1, m\rangle$ by $\Delta_{m}$.

In figure 2.1, the Hamiltonian is graphically shown including a potential tilt due to an external force. The Wannier states $|j, m\rangle$ are localized at a certain cell. The $\Delta_{m}$ terms in the Hamiltonian lead to a coupling of the states with the same band index $m$. The coupling between the bath and the 
vortex is not diagonal in the Wannier basis. Therefore the bath can cause transitions between Wannier states with different band index $m$.

We would like to evaluate the average speed of the vortex

$$
v=\lim _{t \rightarrow \infty} \operatorname{Tr}\{x \dot{\rho}(t)\}
$$

Here $\rho(t)=\operatorname{Tr}_{\text {Bath }} W(t)$ is the reduced density matrix of the system, which is obtained by performing the trace over the bath modes of the density matrix $W(t)$ of the total system. That can be done using real time path integral technique. However this requires that the full Hamiltonian has to be expressed in the eigenstates of the position operator, so called discrete variable representation (DVR). In this DVR basis the position operator is diagonal:

$$
x|j, \mu\rangle=x_{j, \mu}|j, \mu\rangle=\left(j L+x_{\mu}\right)|j, \mu\rangle
$$

The bath Hamiltonian and the Hamiltonian which is due to the external force are already diagonal. To diagonalise the ratchet Hamiltonian $H_{\text {ratchet }}$ we consider the matrix elements of the position operator

$$
\langle j, m|x| j, o\rangle=j L \delta_{m, o}+\xi_{m o}
$$

The matrix $\xi_{m o}$ carries the information of the shape of the potential within the cell, while $j L \delta_{m, o}$ gives the position of the cell. Diagonalization of the matrix $\xi_{m o}$ yields the orthogonal transformation $U$ from the Wannier to the DVR basis $|j, m\rangle=\sum_{\mu} U_{m \mu}|j, \mu\rangle$. The ratchet Hamiltonian in the DVR basis has the following form

$$
\begin{aligned}
H_{\text {Ratchet }} & =\sum_{j=-\infty}^{\infty} \sum_{\mu=1}^{M} \epsilon_{\mu}|j, \mu\rangle\langle j, \mu| \\
& +\sum_{j=-\infty}^{\infty} \sum_{\substack{\nu, \mu=1 \\
\nu \neq \mu}}^{M} \Delta_{\mu, \nu}^{\text {intra }}|j, \mu\rangle\langle j, \nu| \\
& +\sum_{j=-\infty}^{\infty} \sum_{\nu, \mu=1}^{M} \Delta_{\mu, \nu}^{\text {inter }}(|j, \mu\rangle\langle j+1, \nu|+| j+1, \mu\rangle\langle j, \nu|)
\end{aligned}
$$

where $\epsilon_{\mu}=\sum_{m} E_{m} U_{m \mu} U_{m \mu}$ is the on-site energy. The elements $\Delta_{\mu, \nu}^{\text {intra }}=$ $\sum_{m} E_{m} U_{m \mu} U_{m \nu}$ are related to vibrational modes within the cell and $\Delta_{\mu, \nu}^{\text {inter }}=$ 
$\sum_{m}\left(\Delta_{m} / 4\right) U_{m \mu} U_{m \nu}$ allows transitions to the neighboring cell. Figure 2.2 shows a graphical representation of the ratchet Hamiltonian (2.11) in the DVR basis.

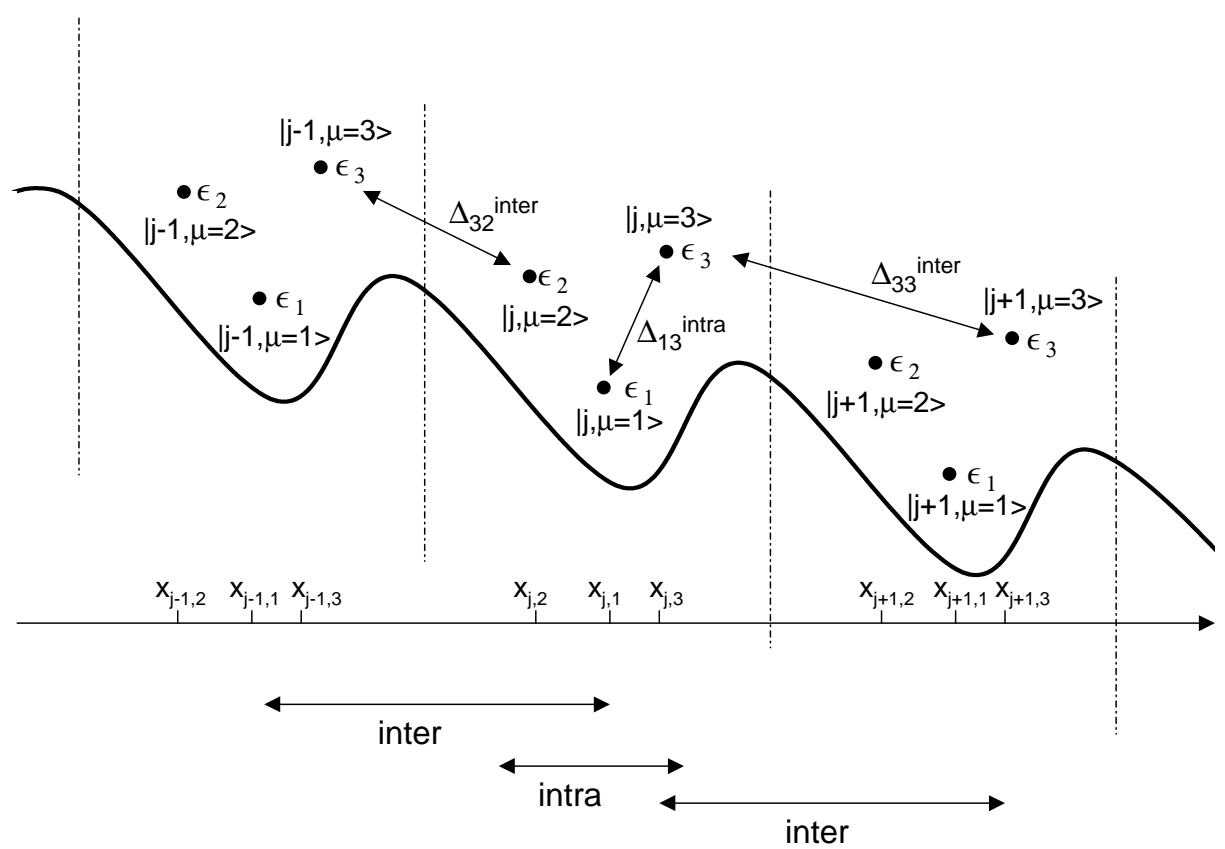

Figure 2.2: Graphical representation of the ratchet Hamiltonian (2.11) in the discrete variable representation (DVR) basis. The DVR states $|j, \mu\rangle$ are centered at a position $x_{\mu}$ in the cell $j$ and have the on-site energy $\epsilon_{\mu}$. The coupling elements $\Delta_{\mu, \nu}^{\text {intra }}$ allow transitions within the cell and $\Delta_{\mu, \nu}^{\text {inter }}$ to the neighboring cell.

The expression for the vortex speed (2.8) reads now

$$
v=\lim _{t \rightarrow \infty} \sum_{j=-\infty}^{\infty} \sum_{\mu=1}^{M} x_{j, \mu} \dot{P}_{i, \mu}(t)
$$

where $P_{i, \mu}(t)$ are the diagonal elements of the reduced density matrix in the DVR basis. They represent the probability of finding the vortex in the state $|j, \mu\rangle$ at the time $t$. If one restricts the dynamics to incoherent tunneling regime, the probabilities obey a master equation 


$$
\begin{aligned}
\frac{d P_{j, \mu}(t)}{d t}= & \sum_{\nu \neq \mu}\left[\Gamma_{\mu, \nu}^{j, j} P_{j, \nu}(t)-\Gamma_{\nu, \mu}^{j, j} P_{j, \mu}(t)\right] \\
& -\sum_{\nu}\left[\Gamma_{\mu, \nu}^{j, j-1}+\Gamma_{\mu, \nu}^{j, j+1}\right] P_{j, \mu}(t) \\
& +\sum_{\nu}\left[\Gamma_{\nu, \mu}^{j-1, j} P_{j-1, \nu}(t)+\Gamma_{\nu, \mu}^{j+1, j} P_{j+1, \nu}(t)\right]
\end{aligned}
$$

where $\Gamma_{\nu, \mu}^{j, j^{\prime}}$ are the transition rates from state $|j, \mu\rangle$ to state $\left|j^{\prime}, \nu\right\rangle$. In the incoherent tunneling regime the transition rates can be approximated as an expansion in the coupling matrix elements $\Delta_{\nu, \mu}^{j, j^{\prime}}$. Here $\Delta_{\nu, \mu}^{j, j}=\Delta_{\mu, \nu}^{\text {intra }}$ and $\Delta_{\nu, \mu}^{j, j \pm 1}=\Delta_{\mu, \nu}^{\text {inter }}$. In lowest order on finds

$$
\Gamma_{\nu, \mu}^{j, j^{\prime}}=\left(\frac{\Delta_{\nu, \mu}^{j, j^{\prime}}}{\hbar}\right)^{2} \int_{-\infty}^{\infty} d \tau e^{-Q_{\mu, \nu}^{j, j^{\prime}}(\tau)} e^{i\left(\epsilon_{\mu}-\epsilon_{\nu}-F L\left(j-j^{\prime}\right)\right) \tau / \hbar}
$$

The influence of the bath is determined by the bath correlation function $[9]$

$$
\begin{aligned}
& Q_{\mu, \nu}^{j, j^{\prime}}(\tau)=\frac{\left(x_{j, \mu}-x_{j^{\prime}, \nu}\right)^{2}}{h} \\
& \quad \times \quad \int_{0}^{\infty} d \omega \frac{J(\omega)}{\omega}\left[\operatorname{coth}\left(\frac{\hbar \omega}{2 k_{B} T}\right)(1-\cos (\omega \tau))+\imath \sin (\omega \tau)\right]
\end{aligned}
$$

Note that the dissipation strength depends on the distance $x_{j}-x_{j}^{\prime}$ between the DVR states.

The stationary solutions of the master equations (2.13) can be found with a Laplace transformation. The vortex speed then assumes the form

$$
v=L \sum_{\nu, \mu} p_{\nu}^{\infty}\left(\Gamma_{\nu, \mu}^{\text {inter }, f}-\Gamma_{\nu, \mu}^{\text {inter }, b}\right)
$$

Here we introduced the forward $\Gamma_{\nu, \mu}^{\text {inter, } f}=\Gamma_{\nu, \mu}^{j, j+1}$ and the backward rate $\Gamma_{\nu, \mu}^{\mathrm{inter}, b}=\Gamma_{\nu, \mu}^{j, j-1}$. The stationary occupation probabilities

$$
p_{\mu}^{\infty}=\lim _{t \rightarrow \infty} \sum_{j} P_{j, \mu}(t)
$$


are expressed in terms of the averaged rates. For three energy bands $M=3$, or equivalently three DVR states, one finds

$$
p_{1}^{\infty}=\frac{\Gamma_{2} \Gamma_{3}-\Gamma_{2,3} \Gamma_{3,2}}{\sum_{\nu=1}^{3} \sum_{\gamma>\nu=1}^{3}\left(\Gamma_{\nu} \Gamma_{\gamma}-\Gamma_{\nu, \gamma} \Gamma_{\gamma, \nu}\right)}
$$

where $\Gamma_{\nu}=\sum_{\mu \neq \nu} \Gamma_{\nu, \mu}$ and $\Gamma_{\nu, \mu}=\Gamma_{\nu, \mu}^{\text {inter }, f}+\Gamma_{\nu, \mu}^{\text {inter }, b}+\Gamma_{\nu, \mu}^{\text {intra }}$. Similarly $p_{2}^{\infty}$ and $p_{3}^{\infty}$ can be found by cyclic index permutation in (2.18).

For one band $M=1$ the vortex speed does not depend on the driving direction, i.e. $v(F)=-v(-F)$. This means that the asymmetry of the potential vanishes. This can also be understood by the fact that with only two parameters $E_{1}$ and $\Delta_{1}$ the ratchet Hamiltonian (2.7) cannot distinguish between a symmetric or an asymmetric sample.

\subsection{Sample design}

In this section we describe how an asymmetric potential for quantum vortices in Josephson junction arrays can be realized. The arrays consist of a long, narrow network of Josephson junctions arranged in a rectangular lattice (see Fig. 2.3 and 2.4). A scanning electron microscope (SEM) picture of part of the most asymmetric mesoscopic device is shown in Fig. 2.4.

Applying a magnetic field perpendicular to the array induces vortices in the system. The number of vortices in the array is equal to the number of flux quanta $\left(\Phi_{\text {tot }} / \Phi_{0}\right)$ applied to the total array [10]. Therefore the onedimensional vortex density $n$ (or equivalently the number of vortices per column) is equal to

$$
n=\frac{\Phi_{\text {tot }}}{\Phi_{0}} \frac{a}{L}
$$

The superconducting current and voltage electrodes along the length of the array (called busbars) repel the vortices, which consequently are forced to move along the centre row. The vortex behaves as a particle with small mass in a one-dimensional potential. For a regular array, as sketched in Figure 1, the potential is approximately cosine-shaped [11] (see Fig. $2.7 \mathrm{~b}$ left). The amplitude of the potential variation is proportional to the Josephson coupling energy $E_{\mathrm{J}}=\frac{I_{c} \Phi_{0}}{2 \pi}$ (with $I_{c}$ the junction critical current ). 


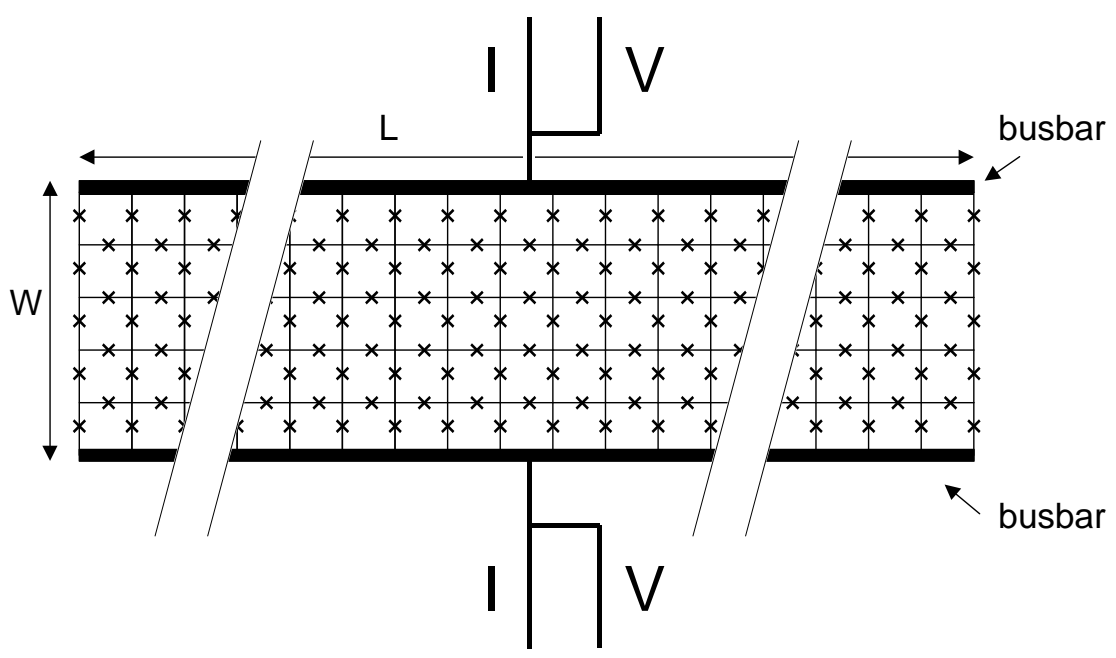

Figure 2.3: Schematic lay out of a Josephson junction array for one-dimensional vortex motion. Josephson junctions are represented by a cross, cells are areas enclosed by four junctions. The arrays have a length of 303 cells and a width of 5 cells between solid superconducting electrodes (busbars). Vortices are induced by an applied magnetic field perpendicular to the array. They are repelled by the busbars and forced to the middle row. Current is applied vertically in the figure between the busbars, homogeneously along the length. The current induces a potential gradient along the length. The motion of vortices through the array creates a voltage across the array.

The mass of the vortex is proportional to the capacitance $C$ of the junctions, $m_{\mathrm{V}} \approx \Phi_{0}^{2} C / 2 a^{2}$ ( $a$ is the lattice constant) [12]. Both parameters, potential $\mathrm{E}_{J}$ and mass $m_{\mathrm{V}}$, can be controlled by fabrication parameters. A vortex of low mass in a weak pinning potential shows the dynamics of a quantum mechanical particle $[13,14,15]$. In these junction arrays, the quantum regime is reached when the charging energy $E_{c}=e^{2} / 2 C$ is of the order of the amplitude of the potential. By injecting a bias current into the busbars a force can be exerted on the vortex. Dissipation by transfer of vortex kinetic energy to oscillatory modes of the junction lattice and to the electromagnetic environment leads to a frictional force. The vortex current (number of vortices passing per unit time) can be determined by measuring the voltage 


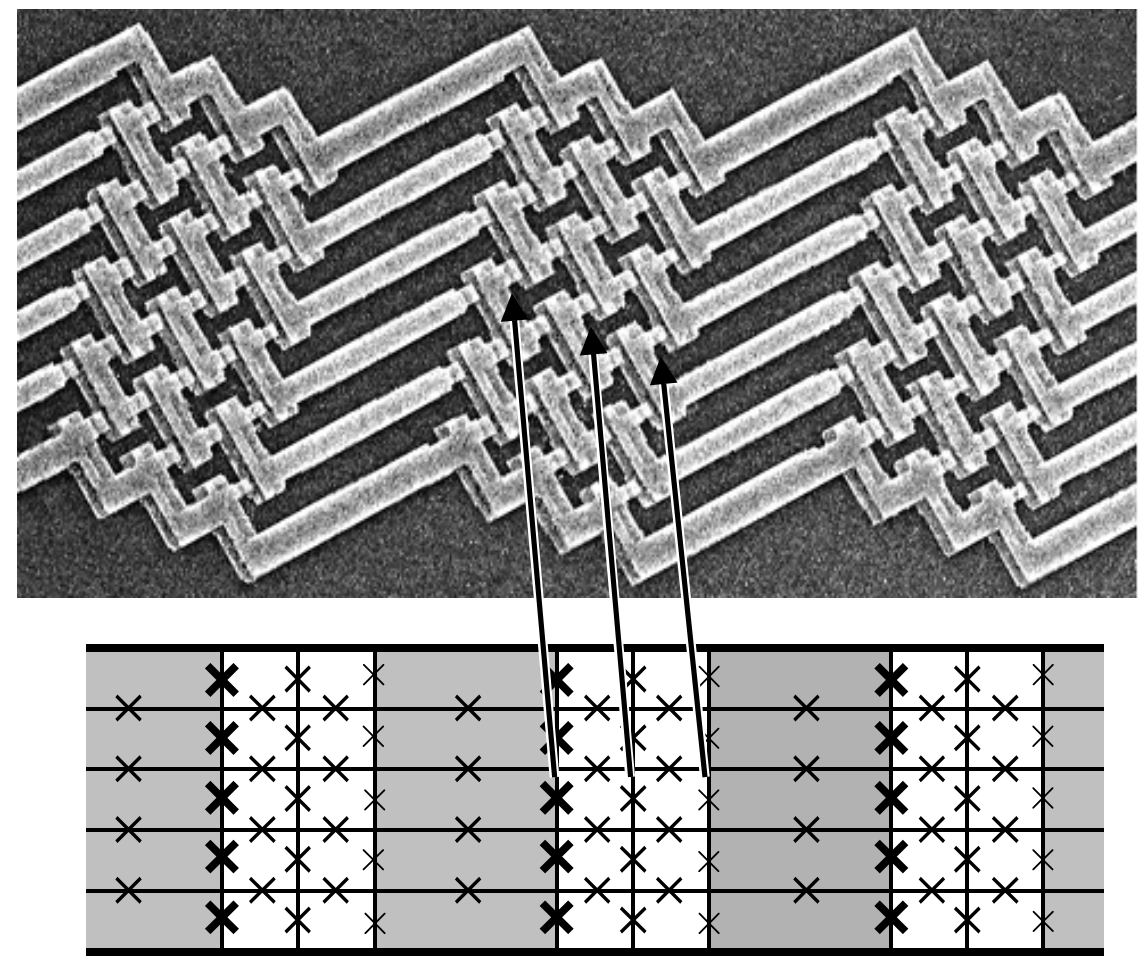

Figure 2.4: Strongly asymmetric array that exhibits ratchet effects. Top: scanning electron microscope picture. Bottom: schematic lay-out. Cells have different areas of $2.52 \mu \mathrm{m}^{2}$ (dark shaded) and $0.84 \mu \mathrm{m}^{2}$. Junctions indicated by arrows have areas of $240 \times 100 \mathrm{~nm}^{2}, 200 \times 100 \mathrm{~nm}^{2}$ and $160 \times 100 \mathrm{~nm}^{2}$ respectively. Vortices have lower energy in cells with larger area and smaller junctions.

between the busbars (2.1). By varying the cell areas or the junction sizes the potential can be modified. Increasing the cell area increases the magnetic flux in the cell, which results in a lowering of the potential minimum. Modifying the junction size changes the Josephson energy and the height of the barrier for vortex motion between cells. These two methods allow for a tailored design of the potential. The strength and symmetry of the potential can be accurately controlled.

We have designed, fabricated and investigated four arrays with identi- 
cal average properties (Fig. 2.7). Three of them are superlattices where a sequence of three cells is repeated along the length of the array. We refer to this set of three cells as the supercell with a length $L$. The devices are fabricated from aluminium on a silicon substrate using shadow evaporation techniques. Sample I (regular) is an array with all cell areas equal to $1.4 \mu \mathrm{m}^{2}$ and all junction sizes equal to $100 \times 200 \mathrm{~nm}^{2}$. These junctions have a capacitance $C$ of $2 \mathrm{fF}$. The critical current of the junctions is $210 \mathrm{nA}$, which is determined from the normal state resistance using the Ambegaokar-Baratoff relation [16]. The characteristic energy scales are $E_{\mathrm{J}} \approx 10 E_{c} \approx 5 \mathrm{~K} \mathrm{k}$. This regular sample serves as a reference for the other samples; the supercell here consists of three identical basic cells. In sample II (symmetric) the cell size is varied periodically along the length of the array. The areas were chosen as 0.6-1.8-0.6 relative to the regular sample. The five cells across the width of the array all have the same area. The potential is lowered at the large cell and increased at the two smaller cells. Sample III (weakly asymmetric) has an asymmetric supercell. The areas of the cells are 0.6-0.9-1.5 relative to the areas of sample I. As expected, the resulting potential (Fig. $2.7 \mathrm{~b}$ ) is asymmetric. The cell areas of the fourth sample (sample IV, strongly asymmetric) were chosen as 0.6-1.8-0.6 relative to the regular sample, and in addition the width of the vertical junctions varied as 1.2-1-0.8 relative to the reference junction. The resulting potential (Fig. $2.7 \mathrm{~b}$ ) is strongly asymmetric. The four samples were fabricated on the same substrate under identical conditions. In order to account for the superlattice structure of samples II, III and IV we introduce the one-dimensional superlattice density $s$ which count the number of vortices in a supercell.

$$
s=\frac{\Phi_{\text {tot }}}{\Phi_{0}} \frac{a}{L / 3}=3 n
$$

\subsection{Measurements}

Measurements on the four samples were carried out in a dilution refrigerator between $12 \mathrm{mK}$ and $1 \mathrm{~K}$. To suppress non-equilibrium noise, Pi-filters were used at room temperature and copper powder filters at sample temperature.

We measured the small-bias resistance as a function of the applied mag- 
$\stackrel{\text { I }}{\text { regular }}$

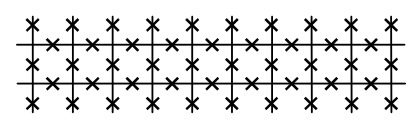

$\mathrm{n}$

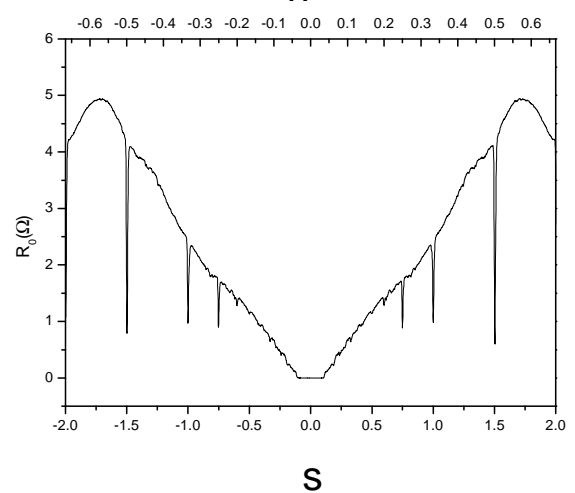

$\mathrm{S}$

III weakly asymmetric
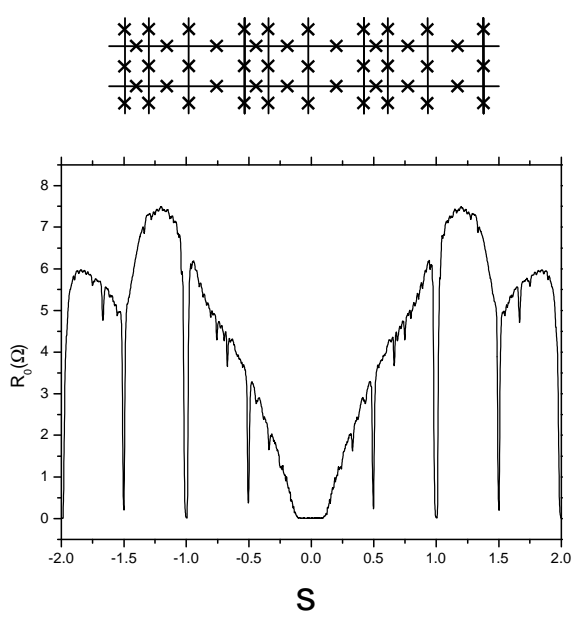

II symmetric
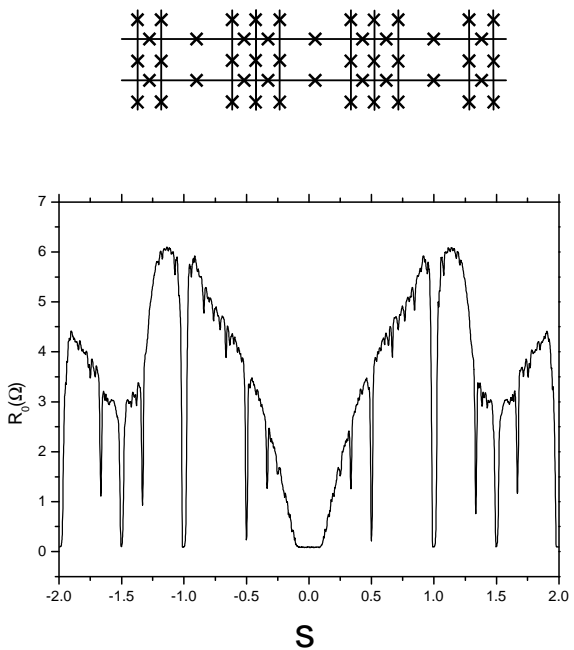

IV strongly asymmetric
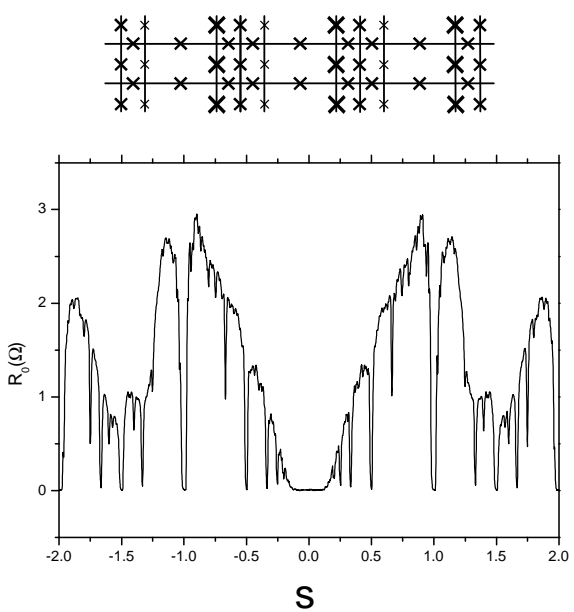

Figure 2.5: Small-bias resistance measurement as a function of the vortex density. Sample I shows localization dips when the one-dimensional density $n$ is equal to $n=1 / N$, where $N$ is an integer. In contrast the superlattice of samples II, III and IV show localization dips at positions when the one-dimensional superlattice density $s=1 / N$, where $N$ is again an integer. 
netic field. This is done by injecting an small oscillatory current of $1 \mu \mathrm{A}_{\mathrm{rms}}$ into the sample. The resulting voltage is measured with a lock-in amplifier at the same frequency as the excitation. This measurement probes the mobility of the vortices at low bias. If the vortices are pinned, the critical current of the vortices is high and the measured resistance is zero. Figure 2.5 shows small-bias resistance for the four samples as a function of the magnetic field. Sample I shows localization dips at magnetic fields where the vortices are commensurate with the underlying array. The dips appear at fields, where the one-dimensional density is one divided by an integer number. In contrast the dips in the measurements of sample II, III and IV appear at positions where the one-dimensional superlattice density is equal to one divided by an integer number. This reflects the superlattice structure of samples II, III and IV.

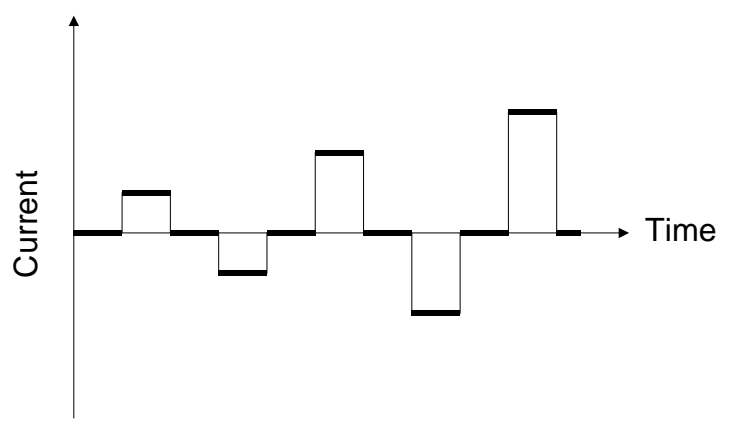

Figure 2.6: Measurement method. A symmetrically alternating block current is applied. This method avoids asymmetry due to the measurement process. The zero current steps between allow the determination of the slow varying voltage offset.

We measured also the dc voltage across the width of the array as a function of the applied bias current (Fig. 2.3). A symmetrically alternating block current was applied to avoid asymmetry of the measurement process. Figure 2.6 shows the current pattern that was applied to the sample. During one block the signal is averaged. The zero-current steps were used to correct for slow voltage drift. The measurement is symmetric for positive and negative currents. 
The ratchet effect manifests itself as a difference between the voltages for positive and negative bias currents. To observe this difference we invert the negative branch of the current-voltage curve with respect to the origin (Fig. $2.7 \mathrm{c}$ ). This method is equivalent to applying a low frequency ac bias (rocking ratchet) and measuring the resulting dc component, but has the advantage that the absolute voltages are known as well.

The measurements show a clear ratchet effect for the strongly asymmetric sample, while no voltage asymmetry is observed between positive and negative current drives for the regular, the symmetric and the weakly asymmetric samples. The symmetry for the regular and the symmetric arrays serves as a check for the validity of the experimental methods. The lack of even weak voltage asymmetry for sample III is remarkable, however. Although the potential is clearly asymmetric, there is no measurable ratchet effect. This can be understood by considering the energy bands of the sample. Sample III has one energy band that is well separated by a gap from the continuum at higher energies (Fig. $2.7 \mathrm{~b})$. Due to the low temperature $\left(12 \mathrm{mK}=0.0024 E_{\mathrm{J}}\right)$ only the single low lying energy band is occupied, as explained in the previous section. This situation is different when more than one band contributes to the transport, like in sample IV. In that case dissipative transitions between energy bands within the supercell occur. The relaxation rates depend on the applied bias. Hence a difference between positive and negative bias can occur and one observes the ratchet effect. It is this interplay between band structure, driving and dissipation which determines the ratchet mechanism in that deep quantum regime ${ }^{1}$.

All voltages depend linearly on the applied magnetic field for average flux up to 0.7 flux quanta per supercell. As the vortex density scales with the flux, this indicates that the interactions between vortices are weak and that the vortices behave as independent particles.

Another signature of quantum behavior is depicted in Fig. 2.8. For classical dynamics and zero temperature the behavior $V \propto I$ is expected above the critical current. However, all of the four samples exhibit a powerlaw behavior $V \propto I^{\delta}$ with exponent $\delta>1$. For a structure like sample I

\footnotetext{
${ }^{1}$ Note that for our few band systems the semiclassical approach in reference [6] cannot be applied for quantitative analysis. However it can still be used for qualitative predictions.
} 

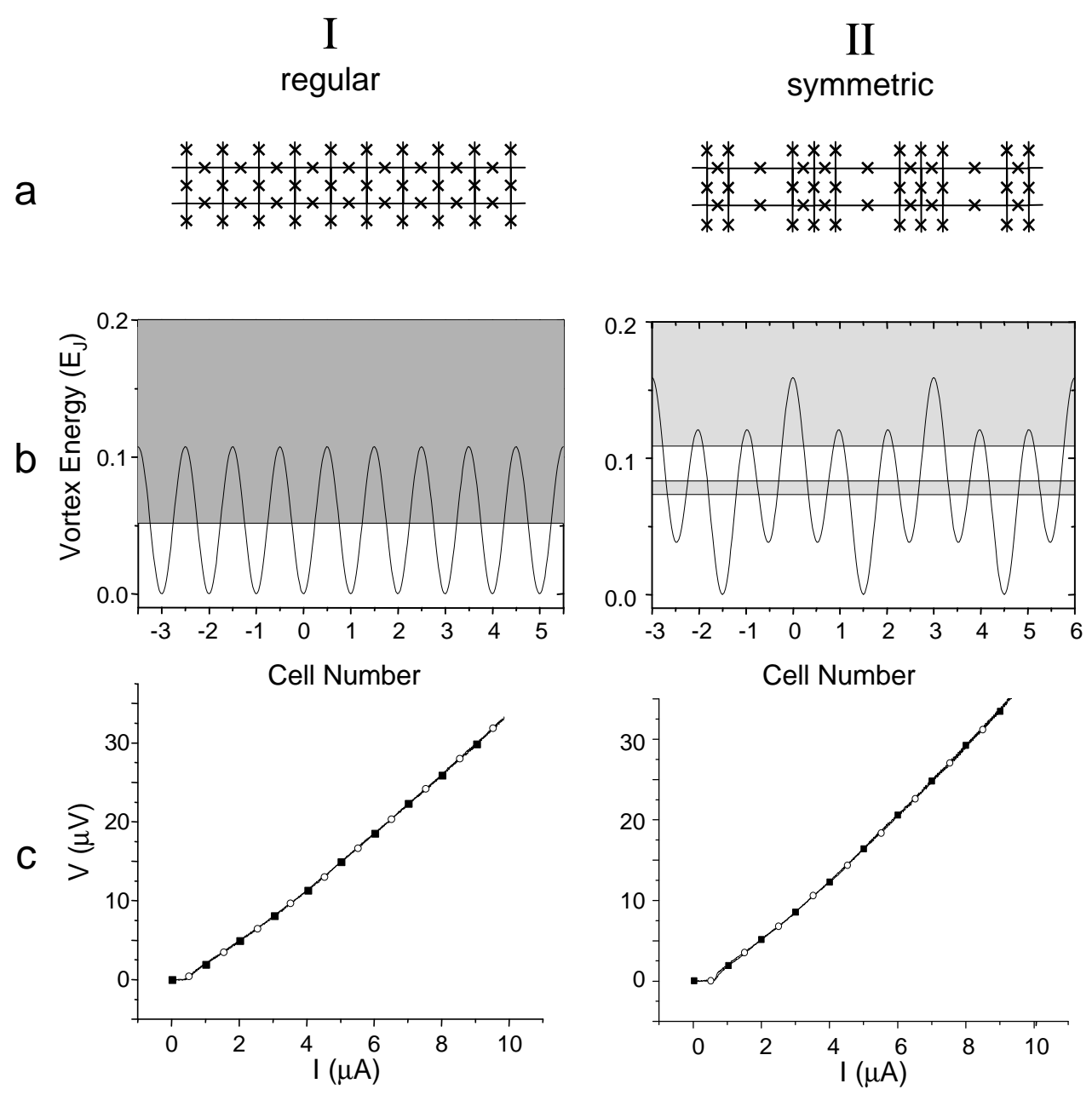

Figure 2.7: Samples and measurement results. The top row (a) indicates three supercells for each of the three arrays, row (b) the resulting potential. The calculated vortex bands are also indicated. Sample I is a regular array as outlined in Fig. 2.3. The cell areas in sample II (symmetric)are periodically varied in a sequence 0.6-1.8-0.6 and in sample III (weakly asymmetric) in a sequence 0.6-0.9-1.5. In sample IV (strongly asymmetric) the areas vary as 0.6-1.8-0.6 and the junction areas as 1.2-1-0.8. The supercells of three basic cells have equal areas in the three samples. Sample I has a cosine-shaped vortex potential and one energy band that connects with a continuum. Sample II shows a weak symmetric modulation on top of of the cosine potential, which leads to a gap in the spectrum with one band below the continuum. ... 
III weakly asymmetric

a

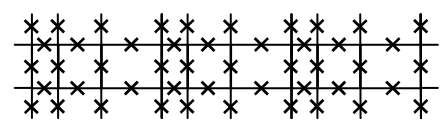

b
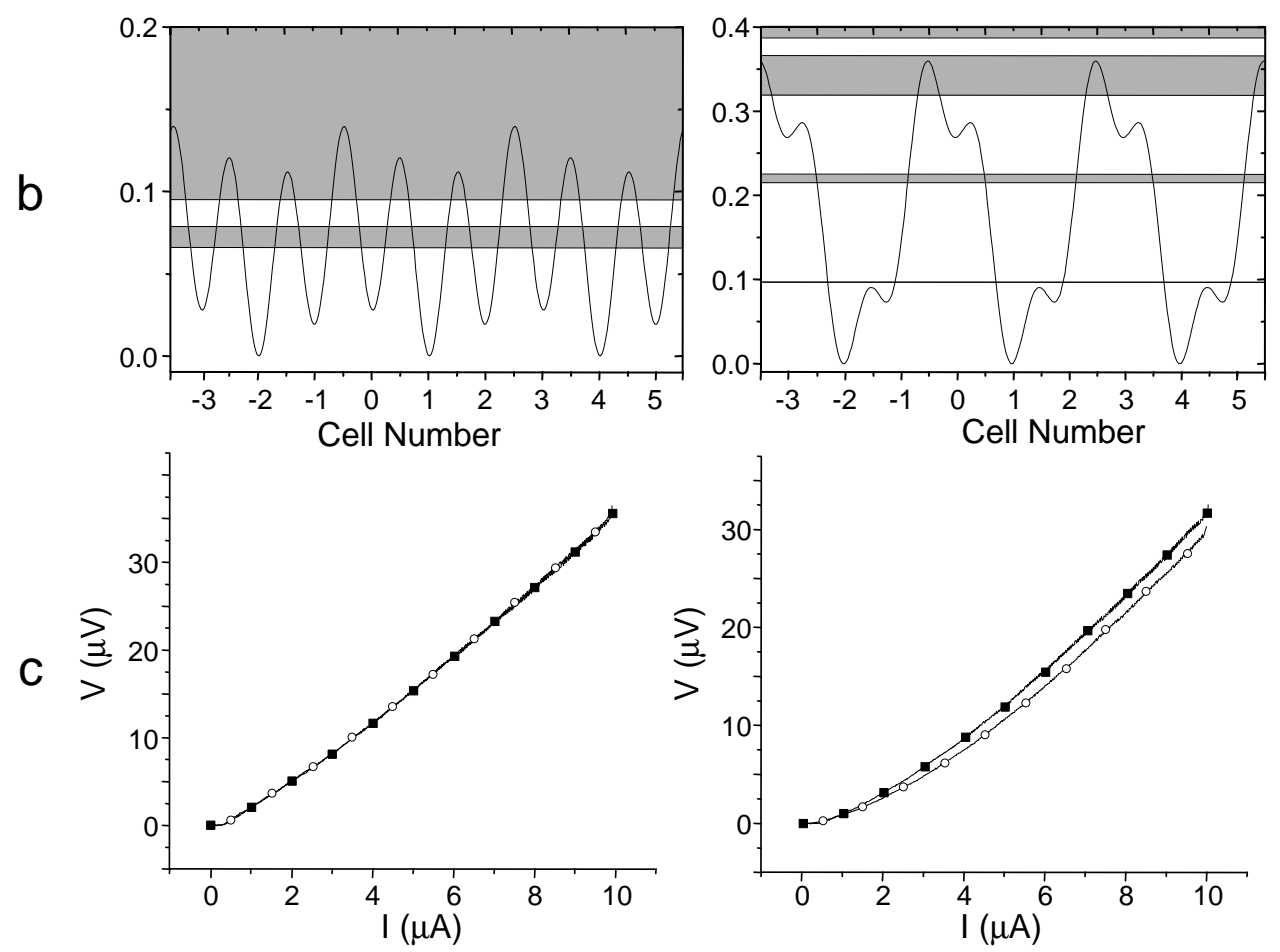

IV strongly asymmetric
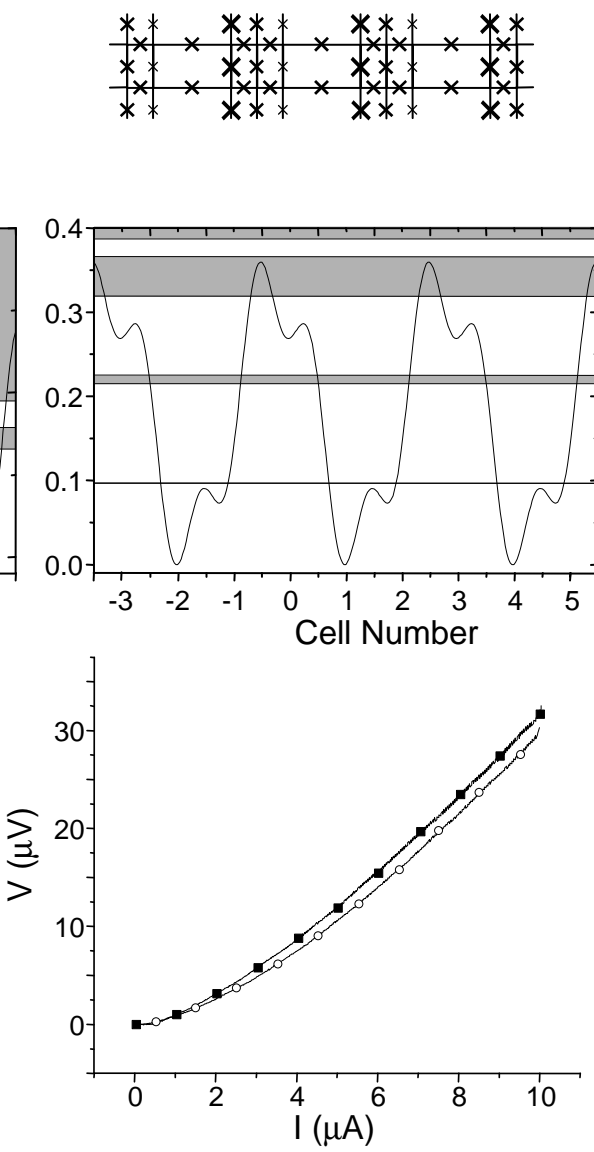

Figure 2.7: ... The potential of sample III shows a weakly asymmetric modulation. The energy bands are similar to sample II: a single energy band which is separated by a gap from the continuum. Sample IV has a strongly asymmetric potential. It shows three energy bands below the continuum, where the lowest band is very narrow. The bottom row (c) gives the measurement results for voltage (vortex current) versus bias current (vortex force), performed at $12 \mathrm{mK}$ and a density of 0.6 vortices per supercell. Open circles label the positive branch and closed squares the negative branch. Sample IV shows a clear asymmetry that is absent in the other three. 


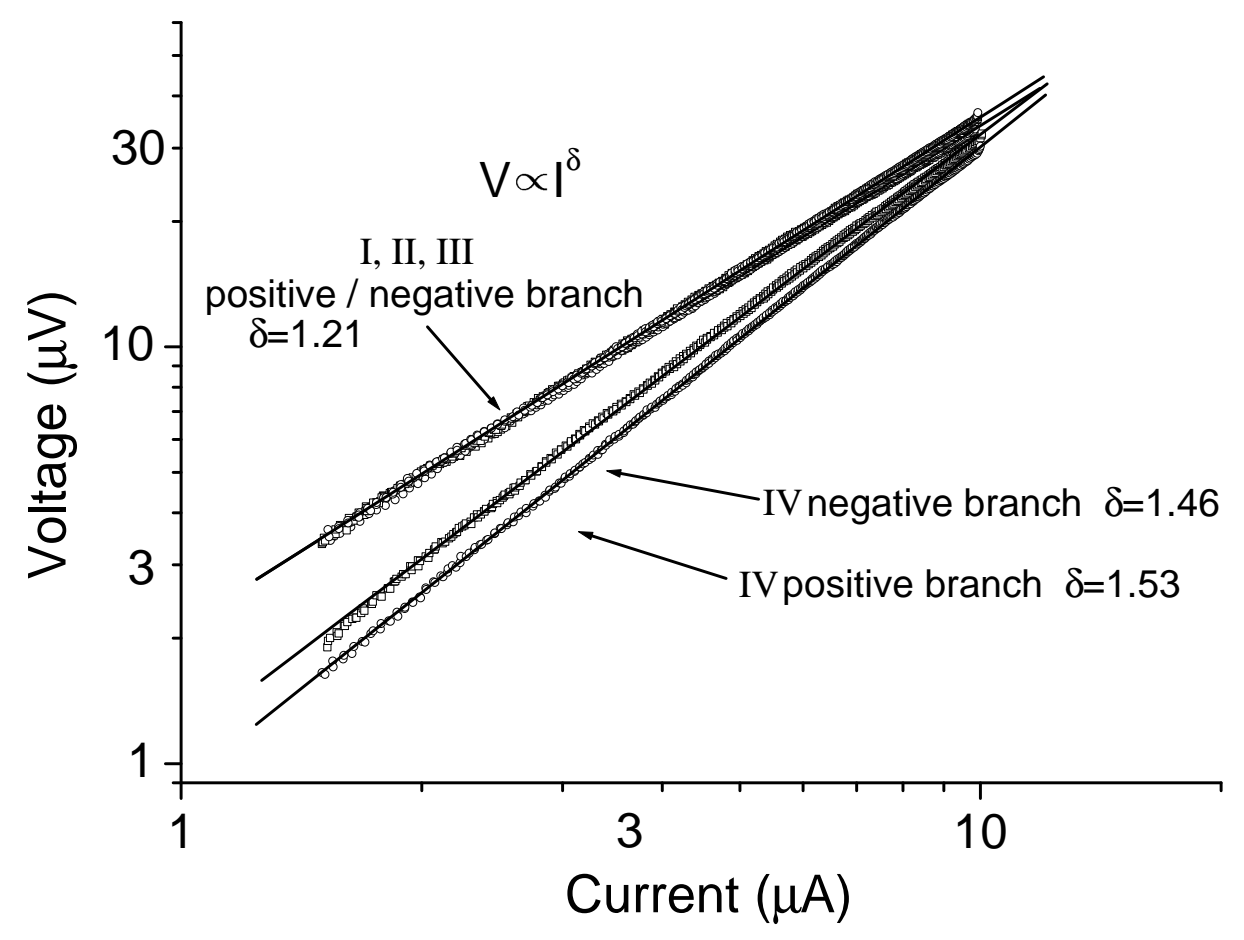

Figure 2.8: Power-law dependence of the $V-I$ characteristics. All four samples exhibit above $1.5 \mu \mathrm{A}$ a behavior $V \propto I^{\delta}, \delta>1$. Because three energy bands are involved in the dynamics, sample IV shows a a larger power than samples I, II and III. The classical behavior would correspond to linear $V-I$ characteristics, i.e., $V \propto I$.

with only one energy band, in the strong current regime $\mathrm{Fa} / \mathrm{K} \gg k_{\mathrm{B}} T$, the forward rate assumes the form $\Gamma^{f} \propto\left(K F L / \pi V_{0}\right)^{2 K-1}[9]$. Here $V_{0}$ is the barrier height of the unbiased potential and $K=\eta a^{2} / h$ is the dimensionless parameter. From the data, a value $K=1.1$ is extracted. The same power is observed in sample II and sample III, and is attributed to the supersymmetric nature of a single band [18]. For sample IV, the situation is different because the higher bands contribute to the dynamics. We measure a higher power for both branches compared to the previous two samples. Furthermore the powers for the positive and the negative branches are slightly different. The increase in the exponent is due to the fact that, in contrast to samples I, 
II and III, the average tunneling distance in sample IV is larger than the average junction distance $a$. Hence the current is more suppressed. The different powers for the two branches reflect the asymmetry of the sample and are in good agreement with numerical simulations [19].

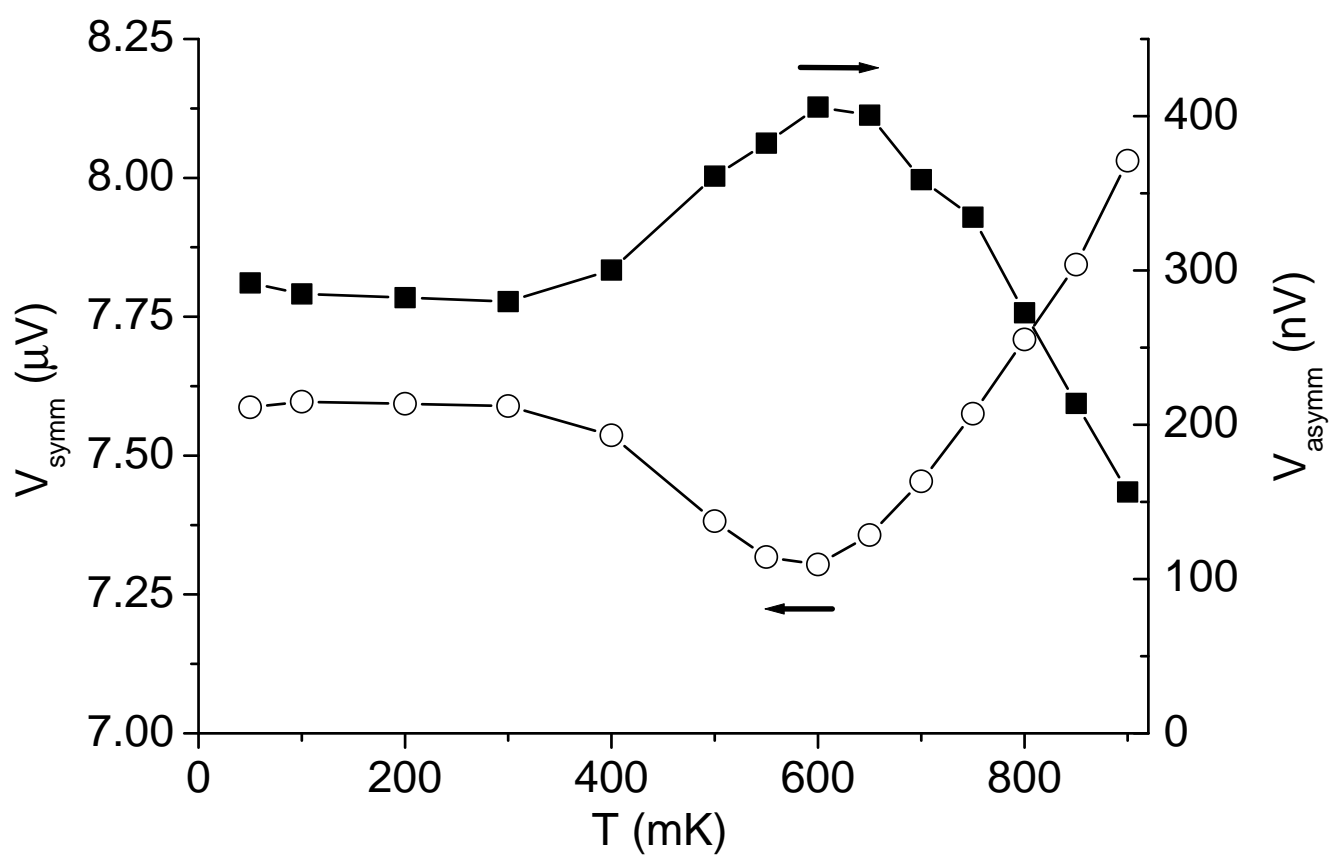

Figure 2.9: Temperature dependence of the ratchet effect. Plotted with closed symbols on the right scale: $V_{\text {asymm }}$ defined as the difference between the voltages for negative and positive currents. Plotted with open symbols on the left scale: $V_{\text {symm }}$ defined as the mean of these voltages. The measurement is performed at a density of 0.28 vortices per supercell and a bias current of $6 \mu \mathrm{A}$. From base temperature to $350 \mathrm{mK}$ no change occurs. Then the asymmetry increases while the average vortex velocity (proportional to $V_{\text {symm }}$ ) decreases. Above $600 \mathrm{mK}$ the vortex velocity increases and the asymmetry diminishes.

Figure 2.9 shows the temperature dependence of the ratchet signal. On the right axis the difference between the two branches $V_{\text {asymm }}=\frac{V(+I)-(-V(-I))}{2}$ is plotted with square symbols for a fixed bias of $6 \mu \mathrm{A}$. We also include the mean of the two branches $V_{\text {symm }}=\frac{V(+I)+(-V(-I))}{2}$ (plotted with round sym- 
bols on the left axis) which shows the mean vortex speed. Below $350 \mathrm{mK}$ down to the base temperature of $12 \mathrm{mK}$ the signals stay constant. This is in accordance with the theory [6], which predicts that the quantum ratchet effect persists down to zero temperature. In contrast, a classical ratchet effect resulting from thermal activation should disappear at low temperatures. Above $350 \mathrm{mK}$ the ratchet signal increases up to $650 \mathrm{mK}$ and then decreases for higher temperatures. The decrease of the signal for temperatures above $650 \mathrm{mK}$ is due to the reduction of the Josephson energy that sets in when the critical temperature of the superconductor is approached, in combination with the increase of the thermal energy. Due to the weaker potential the asymmetry becomes less important and the ratchet effect decreases. The fact that the mean transport (curve with open symbols in Fig. 2.9) increases is consistent with that picture. The increase of the ratchet effect in the intermediate regime $(350 \mathrm{mK}-650 \mathrm{mK})$ is due to the generation of quasiparticles. These quasi-particles are an additional source of friction and cause the ratchet effect to increase [6]. Due to the additional damping the mean transport is reduced as seen in figure 2.9.

To compare the measurements with the theoretical expression of section 2.2 , one has to evaluate expressions (2.1) and (2.16). From the vortex potential the band structure is determined. The energy bands are characterized by their centre energies $E_{1}, E_{2}, E_{3}$ and their bandwidths $\Delta_{1}, \Delta_{2}, \Delta_{3}$. The only two parameters of the theory which are not fixed are the friction coefficient $\eta$ and the cut-off frequency $\omega_{D}$. Figure 2.10 shows preliminarily results of the theoretical calculations. The theory predicts a ratchet effect for this three band system. The positive branch is reproduced well in the range between 0 and $1.3 \mu \mathrm{A}$. Above $1.3 \mu \mathrm{A}$ the theoretically predicted current decreases, which is an artifact due to limitations of the theory, e.g. the truncation to the first $M$ bands. The calculation for the negative branch does not fit the measured data as well as for the positive branch. The theoretical result is about a factor two lower than the measurement. However all these theoretical results are very preliminary. Work is in progress to better understand the calculation and improve the correspondence between measured data and theoretical calculations. Furthermore the temperature dependence has to be investigated. 


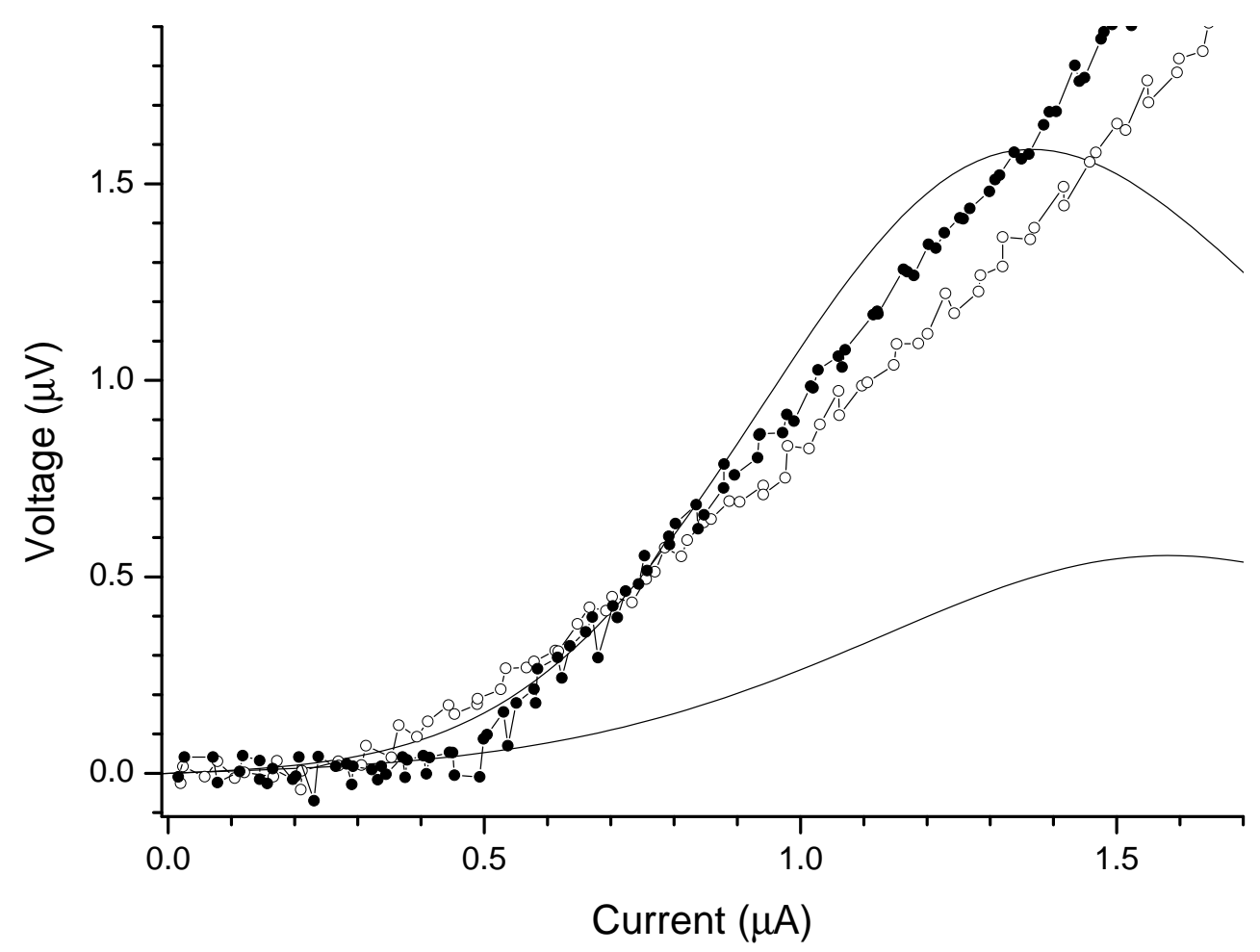

Figure 2.10: Preliminarily theoretical results. The measured data is plotted with round symbols, for the positive branch with open symbols and closed symbols for the negative branch. The theoretical results are shown as a solid line.

\subsection{Conclusions}

In summary, we show that the band structure plays an important role for a quantum ratchet. We develop a theory to model the vortex transport in a ratchet potential with only few energy bands. We find experimentally as well as theoretically that for an asymmetric periodic potential with only one relevant energy band the ratchet effect is missing. This is a consequence of time reversal symmetry. However for a sample with three energy bands we measure a ratchet effect, which originates from the interplay between dissipation and driving. The voltage-current measurements show a perfect power law dependence for all the four samples, which confirms the quantum 
nature of the vortex transport. Preliminarily results from theory predict a ratchet effect. The positive branch is well reproduce for low currents, but the prediction for the negative branch is a factor of two lower than the measured data. Above $350 \mathrm{mK}$ quasi-particles are generated. These quasi-particles lead to an extra source of friction. We observe that this additional friction increases the ratchet effect.

We thank P. Hadley, P. Hänggi for discussions, A. van den Enden and R. Schouten for technical assistance. This work was supported by the Dutch Foundation for Fundamental Research on Matter (FOM).

\section{References}

[1] P. Reimann, "Brownian motors: noisy transport far from equilibrium," Physics Reports, 361, 57 (2002).

[2] Appl. Phys. A 75 (2002), special issue on "Ratchets and Brownian motors: basics, experiments and applications".

[3] J. Maddox, "Making models of muscle contraction," Nature 365, 203 (1993).

[4] J. Maddox, "Directed motion from random noise", Nature 369, 181 (1994).

[5] R. D. Astumian, "Thermodynamics and Kinetics of a Brownian Motor," Science 276, 917 (1997).

[6] P. Reimann, M. Grifoni, P. Hänggi, "Quantum Ratchets," Phys. Rev. Lett. 79, 10 (1997).

[7] H. Linke, T. E. Humphrey, A. Löfgren, A. O. Sushkov, R. Newbury, R. P. Taylor, and P. Omling, "Experimental Tunneling Ratchets," Science 286, 2314 (1999).

[8] M. Tusveld, "Vortex Motion in a Quantum Ratchet," Final Report, TUDelft (2000).

[9] U. Weiss, "Quantum dissipative systems," World Scientific, Singapore, (1999). 
[10] C. Bruder, L. I. Glazman, A. I. Larkin, J. E. Mooij, A. van Oudenaarden, "Phase transition in a chain of quantum vortices," Phys. Rev. B 59, 1383 (1999).

[11] C. J. Lobb, D. W. Abraham, M. Tinkham, "Theoretical interpretation of resistive transition data from arrays of superconducting weak links," Phys. Rev. B 27, 150 (1983).

[12] U. Eckern, A, Schmid, "Quantum vortex dynamics in granular superconducting films," Phys. Rev. B 39, 6441 (1989).

[13] A. van Oudenaarden, S. J. K. Várdy, J. E. Mooij, "One-Dimensional Localization of Quantum Vortices in Disordered Josephson Junction Arrays," Phys. Rev. Lett. 77, 4257 (1996).

[14] A. van Oudenaarden, J. E. Mooij, "One-Dimensional Mott Insulator Formed by Quantum Vortices in Josephson Junction Arrays," Phys. Rev. Lett. 76, 4947 (1996).

[15] T. P. Orlando, J. E. Mooij, H. S. J. van der Zant, "Phenomenological model of vortex dynamics in arrays of Josephson junctions," Phys. Rev. B 43, 10218 (1991).

[16] V. Ambegaokar, A. Baratoff, "Tunneling Between Superconductors," Phys. Rev. Lett. 10, 486 (1963).

[17] G. H. Wannier, "Wave Functions and Effective Hamiltonian for Bloch Electrons in an Electric Field," Phys. Rev., 117, 432 (1960).

[18] P. Reimann, "Supersymmetric Ratchets," Phys. Rev. Lett. 86, 4992 (2001).

[19] J. Peguiron, M. Grifoni, unpublished. 


\title{
Chapter 3
}

\section{Vortex transport in quasi one-dimensional Josephson junction arrays}

\author{
J. B. Majer, J. E. Mooij
}

We study the transport of vortices in quasi one-dimensional Josephson junction arrays. Due to the busbars, the vortices strongly interact and form a rigid chain. The number of vortices can be controlled one by one down to only 6 vortices in an array of length 303 cells. We show an attempt to lower the interaction between vortices. The confining potential was also lowered and the vortices started escaping through the edge. 


\subsection{Introduction}

In this chapter we present two measurements of the transport of vortices in quasi one-dimensional Josephson junction arrays. The great advantage of these systems is that many parameters, like mass, potential, etc. can be designed (For a review see [1]).

The motion of a vortex in Josephson junction array is similar to the motion of a massive particle in a potential [2]. If all junctions are equal the potential is periodic [3]. The strength of the potential is given by the Josephson energy $E_{J}=I_{0} \Phi_{0} /(2 \pi)$, where $I_{0}$ is the critical current and $\Phi_{0}$ the superconducting flux quantum $\Phi_{0}=h / 2 e$. By locally varying the junctions or the cell size one can modify the potential. The mass of the vortex $m_{v}$ is determined by the capacitance $C$ of the junctions [4]: $m_{v}=\Phi_{0}^{2} C / 2 a^{2}$, where $a$ is the lattice constant of the array. A vortex with a large mass in an array with a large potential behaves like a classical particle. However if the charging energy $E c=e^{2} / 2 C$ is of the order of the amplitude of the potential, the vortex behaves like a quantum particle. Localization due to a periodic potential (Mott localization) and localization due to disorder (Anderson localization) of such quantum vortices have been reported $[7,6]$.

In the first section of this chapter we report on a measurement where the interaction between the vortices is very high. Due to this high interaction the vortices move as a rigid chain through the array. The measurements show that one can control the number of vortices one by one down to only 6 vortices.

In the second section we report on a attempt to reduce the vortex interaction. However while the interaction is lowered, the confinement is also reduced and the vortices escape via the edges.

\subsection{Vortex density of quasi one-dimensional Josephson junction arrays}

We start by summarizing the theoretical results of reference [5] in section 3.2.1. Then we describe fabrication and the measurements, which confirm these theoretical results. 


\subsubsection{Theory}

We consider a quasi one-dimensional Josephson junction array (Figure 3.1). The number of cells $W$ in the width is much smaller than the number of cells $L$ in the length. Vortices in the array are induced by applying a magnetic field perpendicular to the array.

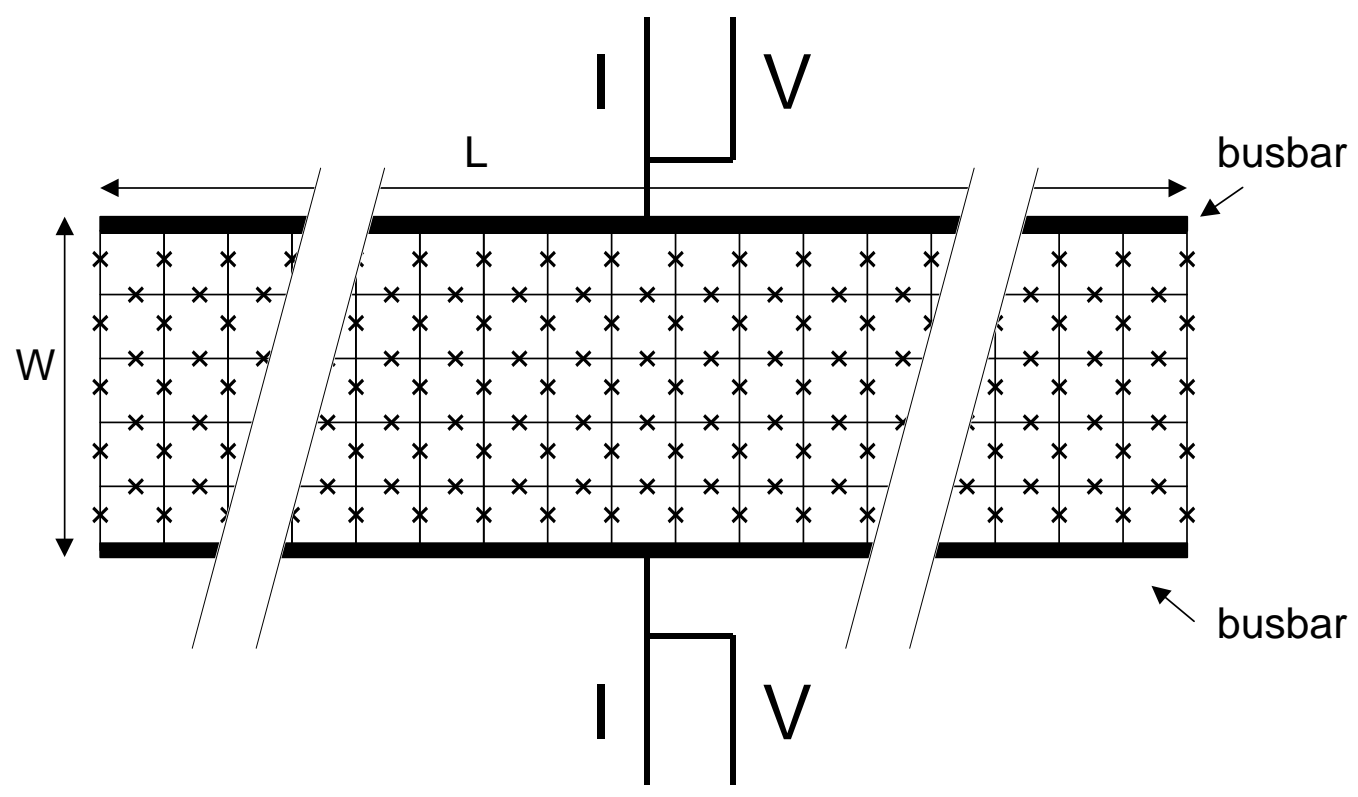

Figure 3.1: Schematic lay out of a Josephson junction array for one-dimensional vortex motion. Josephson junctions are represented by a cross, cells are areas enclosed by four junctions. The array has a length $L$ of 303 cells and a width $W$ of 5 cells between solid superconducting electrodes (busbars). Vortices are induced by an applied magnetic field perpendicular to the array. They are repelled by the busbars and forced to the middle row. Current is applied vertically in the figure between the busbars, homogeneously along the length. The current induces a potential gradient along the length. The motion of vortices through the array creates a voltage across the array.

The busbars along the length of the array repel the vortices and force them to move in the middle row. Furthermore the busbars also lead to a very long range repelling interaction between the vortices. If one neglects 
the effects of the magnetic fields induced by the vortices (discussed in chapter IV of reference [5]), the interaction range is as long as the length of the array. A finite magnetic field now creates a rigid chain of vortices. The number of vortices in the chain is

$$
N=\lfloor n L\rfloor
$$

where $L$ is the number of cells in the length of the array and $n$ the onedimensional density. The function \lfloor\rfloor means rounding towards the nearest lower integer number. The one-dimensional density $n$ is defined by

$$
n=W \frac{B S}{\Phi_{0}}
$$

and therefore equal to the number of superconducting flux quanta applied to one column of the array. Here $B$ is a perpendicular magnetic field, $S$ the area of a cell and $W$ the number of cells in the width.

It is interesting to recognize that $n L\left(n L=B W L S / \Phi_{0}\right)$ is the number of flux quanta applied to the whole array. So the number of vortices in the array is equal to the integer number of flux quanta applied. Similarly $n$ is the one-dimensional density of vortices (vortices per unit length). The distance between the vortices is $d_{\text {vort-vort }}=1 / n$.

The activation energy of the vortex chain is the energy difference between the ground state with $N$ vortices and the state with $N+1$ vortices at a given magnetic field. This energy is the energy barrier due to the boundary of the array

$$
E_{b}(n L)=\frac{\pi^{2} E_{J} L}{2 W} \frac{1}{n L}[1-\operatorname{Frac}(n L)] \operatorname{Frac}(n L)
$$

Here we used the notation $\operatorname{Frac}(n L):=n L-\lfloor n L\rfloor$. The boundary energy vanishes at integer values of $n L$ (see figure 3.2). At these positions the inter vortex distance $d_{\text {vort-vort }}$ is such, that the chain with $N+1$ vortices exactly fits the length of the array. The maxima between the zeros of $E_{b}$ between the two zeros are $\pi^{2} E_{J} /(8 n W)$ and decay with the magnetic field like $1 / n$.

One can also calculate the critical current of the array. The critical current $I_{c}$ is defined as the minimal current needed to start moving the vortex chain 


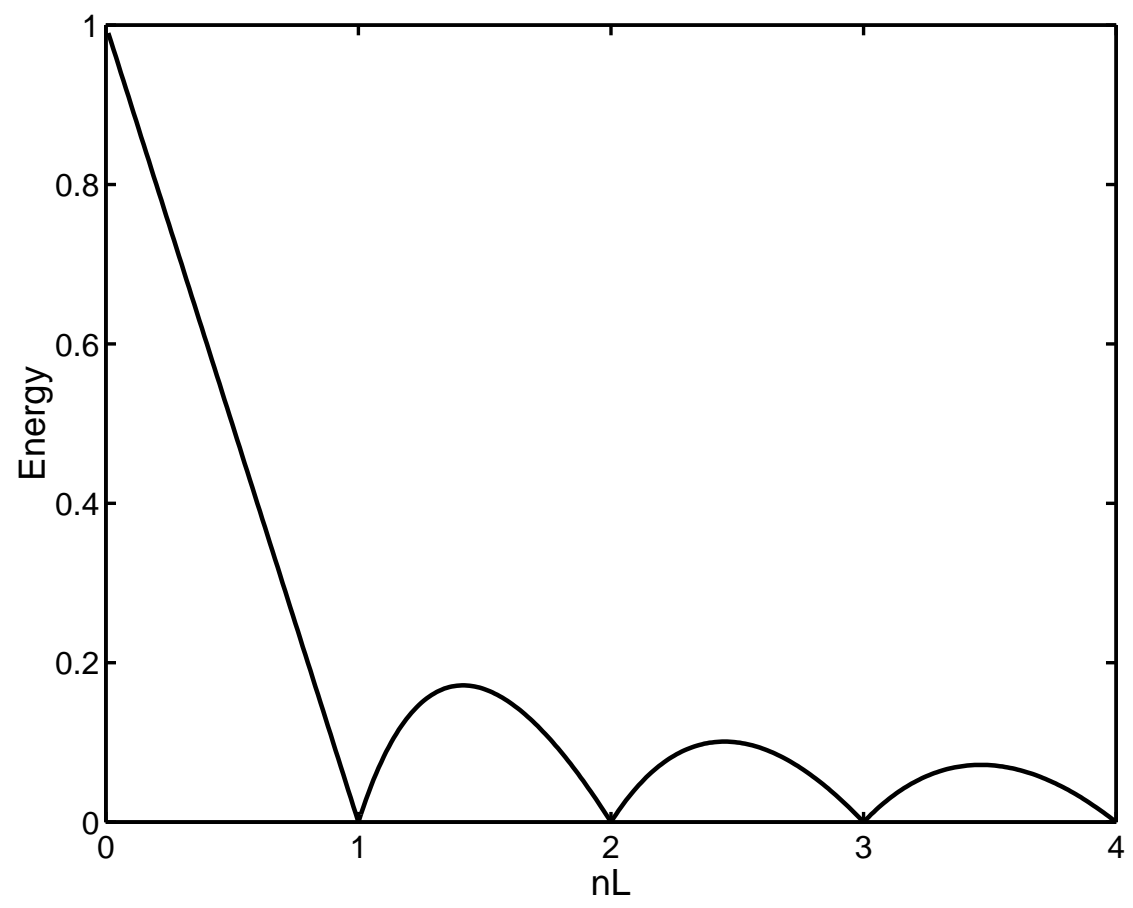

Figure 3.2: Boundary energy in units of $\pi^{2} E_{J} L / 2 W$ of the vortex chain as a function of $n L=W L B S / \Phi_{0}$. The critical current of the array is given by the same curve $I_{c}^{\text {array }}=4 E_{b}(n L) / \Phi_{0}$.

through the array:

$$
I_{c}^{\text {array }}=\frac{\pi I_{c} L}{W} \frac{1}{n L}[1-\operatorname{Frac}(n L)] \operatorname{Frac}(n L)=\frac{4 E_{b}}{\Phi_{0}}
$$

The critical current has the same form as the boundary energy. It is clear that all these considerations break down at higher magnetic fields. When the distance between the vortices becomes too small, the repelling force leads to a zigzag formation of the chain.

\subsubsection{Sample design and measurements}

We have fabricated a sample according to the scheme in figure 3.1. The devices are fabricated from aluminum on a silicon substrate using shadow evaporation techniques as described in the introduction 1.3. The cell area 
is equal to $1.4 \mu \mathrm{m}^{2}$. The width $W=5$ cells is much smaller than the length $L=303$ cells. The junctions have a size of $100 \times 200 \mathrm{~nm}^{2}$. The critical current of the junctions is $210 \mathrm{nA}$, which is calculated from the normal state resistance using the Ambegaokar-Baratoff relation [9]. The Josephson energy is equal to $E_{b}=4.9 \mathrm{~K} \mathrm{k}$.

We measured the small-bias resistance as a function of the applied magnetic field. This is done by injecting a small oscillatory current of $2 \mu \mathrm{A}_{\text {rms }}$ into the sample. The resulting voltage is measured with a lock-in at the same frequency as the excitation. This measurement probes the mobility of the vortices at low bias. If the vortices are pinned, the critical current of the vortices is high and the measured resistance is zero. Figure 3.3 shows small-bias resistance for very small magnetic fields. One observes the peaks in the measurement at positions where $n L$ is integer starting from $n L=6$. At these postitions the critical current $I_{c}$ (Eq. 3.4) is low and the vortex chain has a higher mobility. The peak height becomes smaller as the field decreases. This can be explained by the fact that the maxima of $E_{b}$ vanish like $1 / n$. We could not detect peaks for $n L$ of five or lower. As one can see in figure 3.2 the dips in the activation energy become sharper for smaller fields.

Any experimental imperfection can easily smear out the dips and the peaks in the small bias resistance disappear.

\subsubsection{Conclusion}

Due to the busbars the interaction between the vortices is very high. This leads to rigid chain of vortices in the array. We measure peaks in the mobility of the vortices where $n L$ is an integer. These peaks occur when the chain of $n L$ vortices exactly fits the length of the array. This shows that we have control over the exact number of vortices in the array. We can control down to 6 single vortices in a array of 303 cells where the distance between vortices is more than 50 cells. 


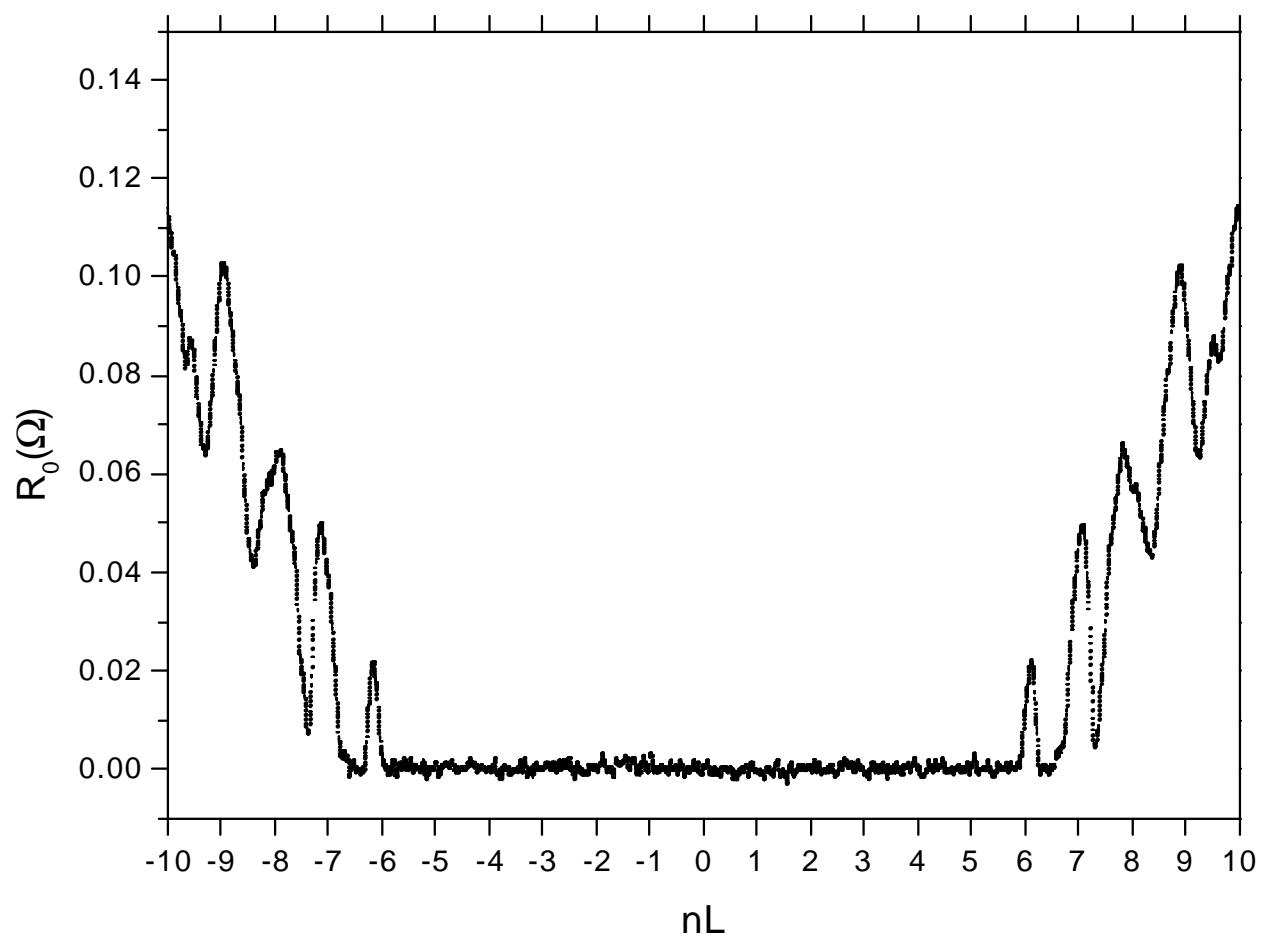

Figure 3.3: Small bias resistance vs magnetic field $n L=W L B S / \Phi_{0}$. An excitation current of $2 \mu \mathrm{A}$ is used. Starting from $n L=6$ one observes peaks in the mobility at integer values of $n L$. 


\subsection{Quantum vortices with weak interaction}

We concluded in the previous section that the busbars lead to very strong interaction. The vortices form a rigid chain. This chain has a large mass and behaves as a more classical object than a single vortex. To observe quantum effects one has to decrease the interaction between the vortices and make the chain less rigid. A practical way to reduce the interaction is to replace the busbars by Josephson junctions. Now a phase gradient along the edge of the array can occur which decreases the interaction range between the vortices. However this decreases also the confining potential across the width of the array. The vortices can now escape via the edge. Furthermore the number of vortices in the array is not proportional to the field and equation 3.1 does not hold. The busbars in the previous design also provided a homogeneous current injection across the length of the array. Now each edge island has to be connected via a series resistor to the current source. The resistance of this series resistors should be considerably larger than the normal state resistance of the Josephson junctions in the array. Experimentally such large resistance values are very hard to realize. Therefore we added an extra row of ten Josephson junctions to the series resistor. The Josephson inductance $L_{J}=\Phi_{0} /\left(2 \pi I_{0}\right)$ of these junctions provides an extra impedance. Figure 3.4 shows the scheme of the array. The junctions on the edge of the array can be made relatively larger than the junctions in the array. This allows us to tune the interaction between the vortices. Voltage probes on both ends of the array detect the vortices.

\subsubsection{Sample fabrication and measurements}

We have designed and fabricated two arrays, which differ in the size of the edge junction. The edge junctions of the first sample have the same size as the junction in the array and the series junctions. Figures 3.5 and 3.6 show scanning electron images of these samples. An animated version can be found in [8]. The second sample has edge junctions which are twice as large as the junctions in the array. The fabrication involves two steps. In the first step a layer of platinum is evaporated. With this platinum layer the

resistors are defined. In the second step the Josephson junctions are made 


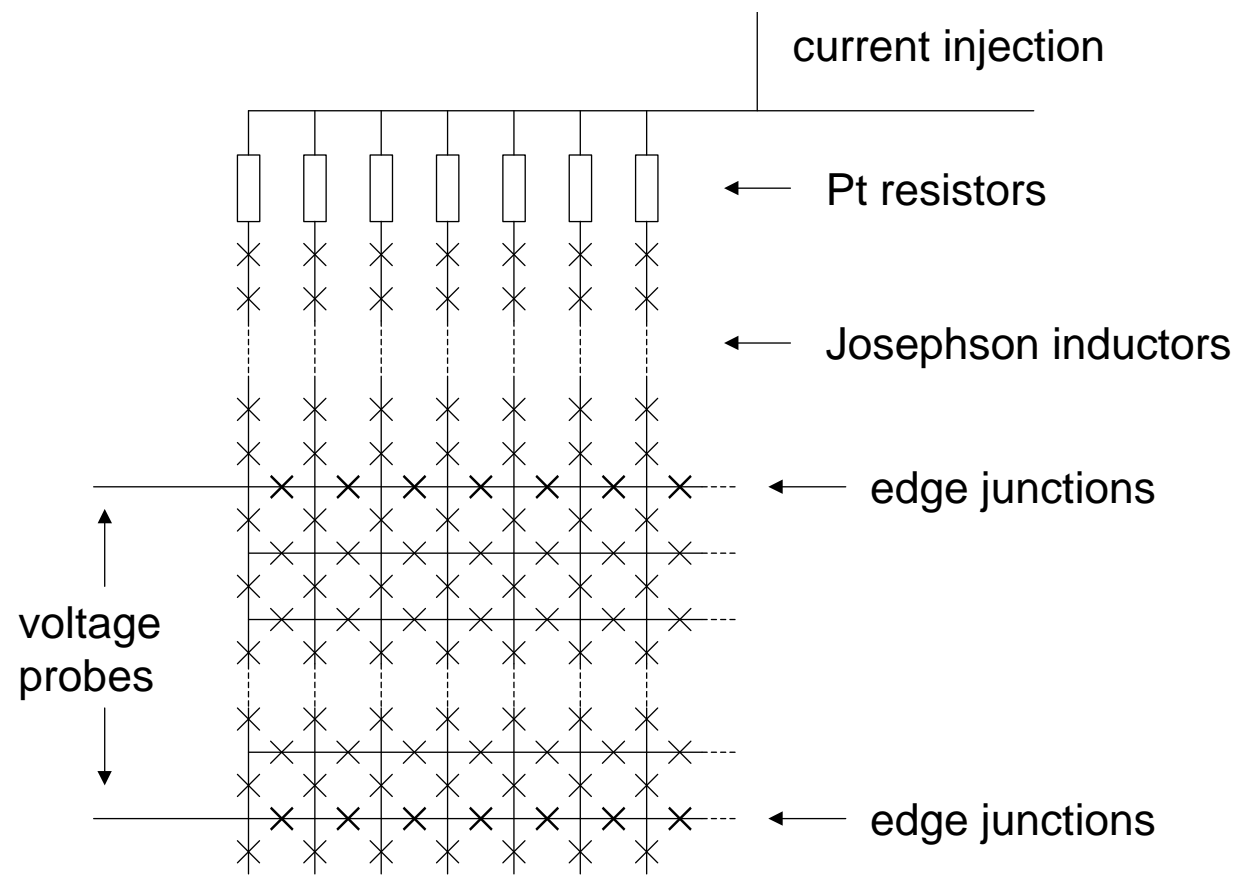

Figure 3.4: Scheme for a quasi one-dimensional Josephson junction array without busbars. To provide a homogeneous current injection parallel series resistors are used. A row of ten Josephson junctions increases the impedance of the series resistor . The array has a width of 7 cells and a length of 999 cells. The relative size of the junctions on the edge of the array defines the vortex interaction and the confining potential. Voltage probes on both ends detect the motion vortices.

by shadow evaporation (see section 1.3). We have taken great care of the homogeneity of the structure by checking the resistance of test circuits on the same substrate. We found that the spread in resistance of the series resistor is smaller than $1 \%$. The room temperature resistance is $2.91 \mathrm{k} \Omega$. One expects this value to decrease by a factor of about two at low temperatures. The normal state resistance of the test junctions has an average value of $890 \Omega$ and a spread of $4 \%$. The critical current of the junctions is $360 \mathrm{nA}$, which is calculated from the normal state resistance [9]. This leads to a Josephson energy of $E_{J}=8.4 \mathrm{~K} \mathrm{k}$. With the area of the junctions of $200 \times 100 \mathrm{~nm}^{2}$ 
we estimate that the junction capacitance is about $2 \mathrm{fF}$. With these values a ratio $E_{J} / E_{c}=18$ follows.

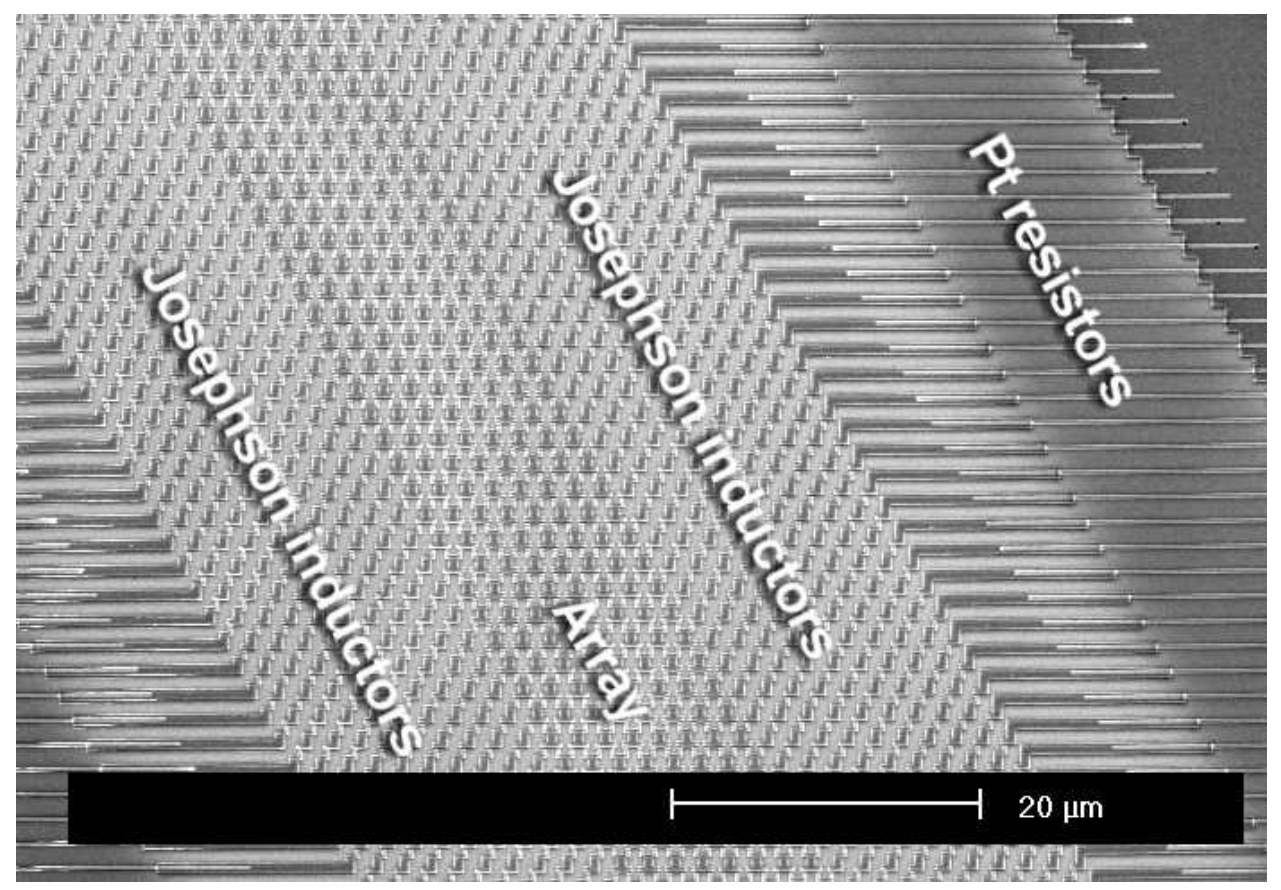

Figure 3.5: Scanning electron microscope image of the array and the series circuit. The platinum resistors are visible as small lines on the right side of the image.

We measured the voltage of the two probes as a function of the applied current. Figure 3.7 shows results for the sample with two times larger edge junctions. The applied magnetic flux in the top figure is $0.189 \Phi_{0}$ and for the bottom figure $-0.059 \Phi_{0}$. One observes that the voltages are different for the two probes at higher currents. A different voltage for the two probes means, that some of the vortices pass through one of the probes and then escape via the edges. The voltage that we measure across the edge confirms that assumption. The confining potential is too weak to keep the vortices in the array. Furthermore the V-I curves are asymmetric. This is due to the remaining spread of junction parameters in the array. Without busbars the number of vortices in the array is not proportional to the magnetic field. Therefore one has to look for special features in the magnetic field dependence 


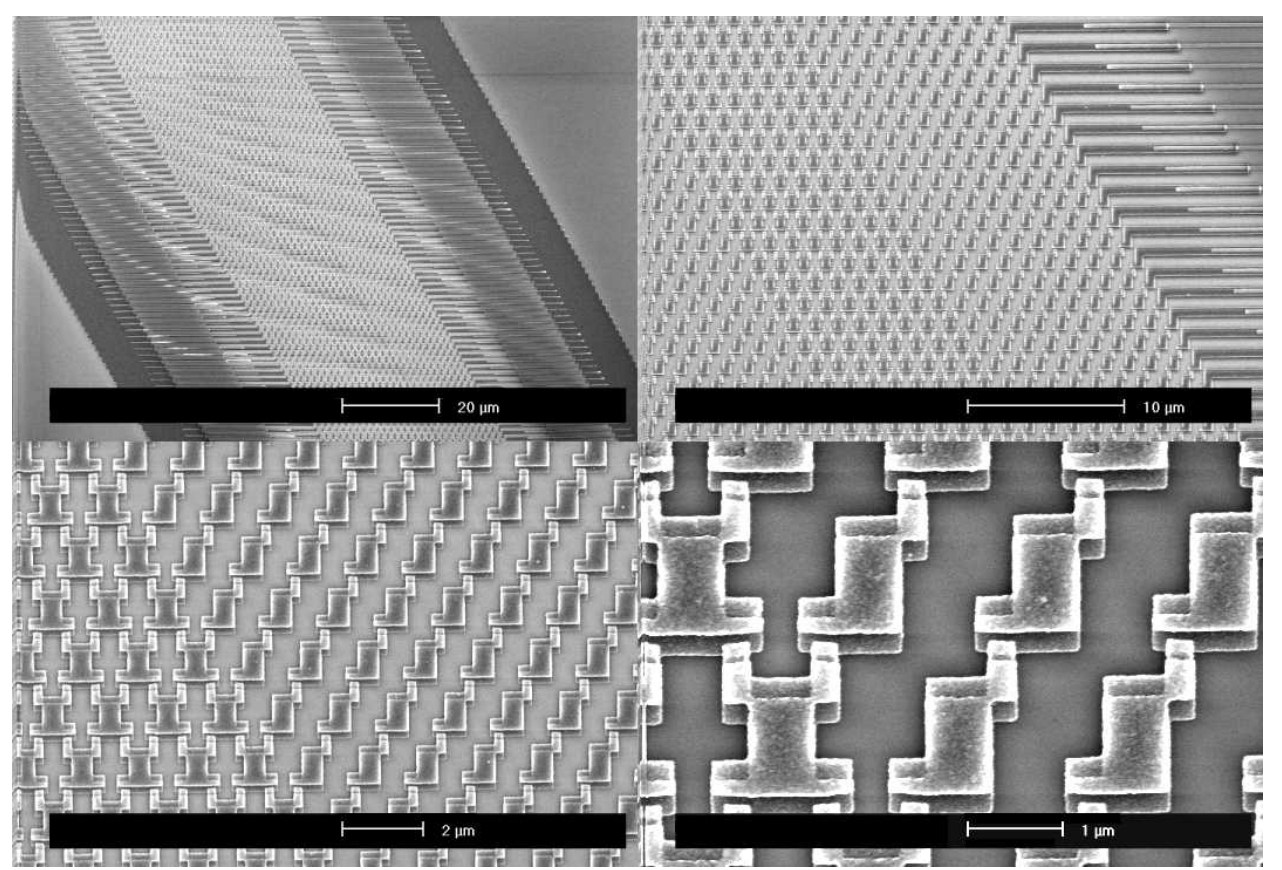

Figure 3.6: Scanning electron microscope images at different magnifications. The bottom images show parts of the array and the series junctions. An animated version of these images can be found in [8].

to relate the density of vortices to the field. However we could not observe any sign of one-dimensional localization as in the samples with busbars [7].

\subsubsection{Conclusions}

We have replaced the busbars with Josephson junctions. This reduces the interaction between the vortices but also the confining potential. A special series circuit with a resistor and a row of ten Josephson junctions provides a homogeneous current injection. We have taken special care about the homogeneity of the resistors and Josephson junctions. Measurements show that the confining potential is weak and that the vortices escape already at low bias currents. The measured voltage-current curves are asymmetric which is due to the remaining spread of junction parameters in the array. We could not observe any localization of vortices which leaves the number of 

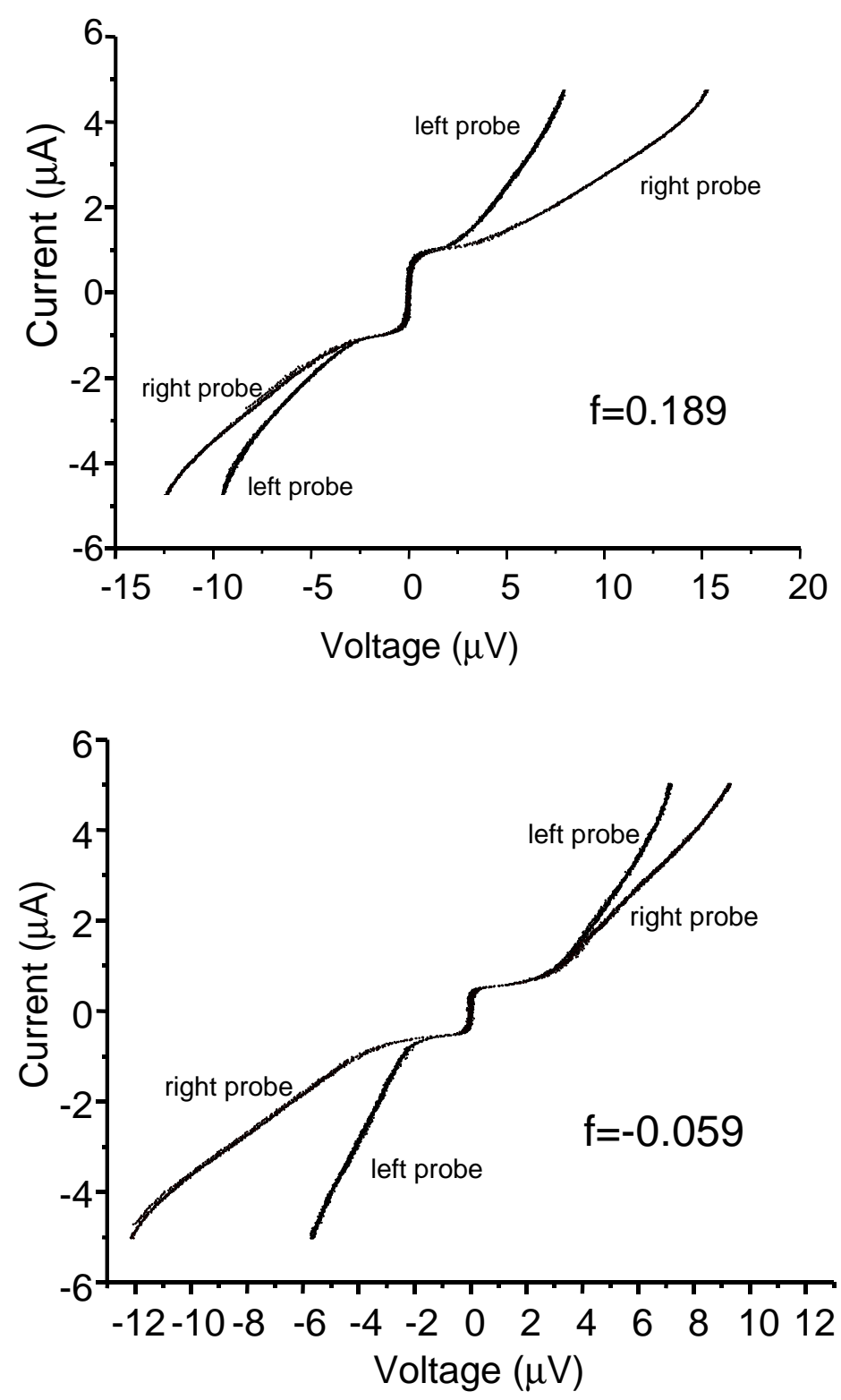

Figure 3.7: Voltage versus current measurements for the two probes. The flux applied is $0.189 \Phi_{0}$ for the top figure and $0.059 \Phi_{0}$ for the bottom figure. The voltages for the two probes are different which indicates that vortices escape via the edges. 
vortices in the array unknown. 


\section{References}

[1] R. Fazio and H. van der Zant, "Quantum phase transitions and vortex dynamics in superconducting networks," Physics Reports, 355, 235 (2001).

[2] T. P. Orlando, J. E. Mooij, H. S. J. van der Zant, "Phenomenological model of vortex dynamics in arrays of Josephson junctions," Phys. Rev. B 43, 10218 (1991).

[3] C. J. Lobb, D. W. Abraham, M. Tinkham, "Theoretical interpretation of resistive transition data from arrays of superconducting weak links," Phys. Rev. B 27, 150 (1983).

[4] U. Eckern, A, Schmid, "Quantum vortex dynamics in granular superconducting films," Phys. Rev. B 39, 6441 (1989).

[5] C. Bruder, L. I. Glazman, A. I. Larkin, J. E. Mooij, A. van Oudenaarden, "Phase transition in a chain of quantum vortices," Phys. Rev. B 59, 1383 (1999).

[6] A. van Oudenaarden, S. J. K. Várdy, J. E. Mooij, "One-Dimensional Localization of Quantum Vortices in Disordered Josephson Junction Arrays," Phys. Rev. Lett. 77, 4257 (1996).

[7] A. van Oudenaarden, J. E. Mooij, "One-Dimensional Mott Insulator Formed by Quantum Vortices in Josephson Junction Arrays," Phys. Rev. Lett. 76, 4947 (1996).

[8] Available on

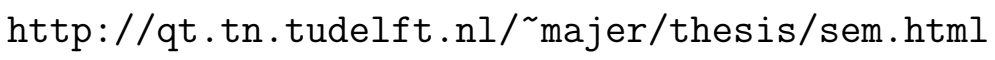

[9] V. Ambegaokar, A. Baratoff, "Tunneling Between Superconductors," Phys. Rev. Lett. 10, 486 (1963). 


\section{Chapter 4}

\section{Simple phase bias for superconducting circuits}

J. B. Majer, J. R. Butcher, F. A. Zwanenburg, J. E. Mooij

A superconducting phase-bias tool, based on a trapped fluxoid in a ring, is proposed and demonstrated. It can provide arbitrary phase values and is simple to fabricate. The phase bias has been realized in two superconducting quantum interference devices, where the critical current versus magnetic flux is shown to be shifted by $\pi / 2$ and $\pi$. Several applications of such a phase bias ring are presented.

Parts of this chapter have been published in Applied Physics Letters 80, 3638. (2002). 


\subsection{Introduction}

Many superconducting devices require an imposed phase difference. Usually this phase bias is applied by means of magnetic flux in a closed superconducting loop. An example is the superconducting quantum interference device (SQUID) [1], which needs a phase bias of around $\pi / 2$. Several types of superconducting quantum bits rely on a phase difference $\pi[2,3,4]$. So-called $\pi$-junctions, where the dependence of the current on the phase difference is shifted by $\pi$, are seen as attractive components in superconducting electronics [5]. $\pi$-junctions have been developed with d-wave superconductors [6] and with superconductor-ferromagnet-superconductor junctions [7]. However these methods involve exotic materials and complicated fabrication methods. We have developed a surprisingly simple phase-bias tool, based on trapped fluxoids in a closed loop without junctions. It can be fabricated very easily from materials such as niobium or aluminum; any phase shift can be realized. Applying the phase bias with a coil can introduce a lot of noise, either by the noise of the current source or by moving vortices in the superconducting coil wire. In contrast the ring is very small and the whole circuit can easily be shielded. Gradiometer designs as presented in section 4.4 can further reduce the noise due to external flux.

\subsection{The phase bias ring}

Our approach relies on a mesoscopic superconducting ring (Fig. 4.1 (a)) with narrow cross section relative to the penetration depth. The phase difference along the circumference $\gamma$ is proportional to the current $I$ in the ring. The proportionality factor is given by the kinetic inductance of the ring $L_{K}$

$$
\gamma=2 \pi \frac{L_{K}}{\Phi_{0}} I
$$

where $\Phi_{0}$ is the superconducting flux quantum. The kinetic inductance of the ring depends on the London penetration depth $\lambda_{L}$ and geometrical factors [8]:

$$
\gamma=\frac{2 \pi}{\Phi_{0}} \oint \mu_{0} \lambda_{L}^{2} \mathbf{J}_{s} d \mathbf{s}
$$


If one assumes a homogeneous current distribution in the wire $J_{s}=I /(h w)$ the kinetic inductance becomes

$$
L_{K}=\mu_{0} \lambda_{L}^{2} \frac{s}{w h}
$$

where $s$ is the total circumference, $w$ the width and $h$ the height of the cross section of the wire.

The kinetic inductance is related to the normal state resistance $R_{n}$ of the wire. For a dirty one-dimensional superconductor the relation is $[1,8]$

$$
L_{K}=\frac{\Phi_{0}}{\pi^{2}} \frac{e R_{n}}{\Delta}
$$

where $\Delta$ is the superconducting gap and $e$ the electron charge.

(a)

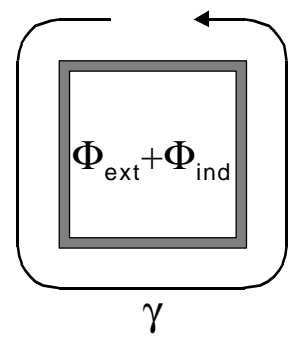

(b)

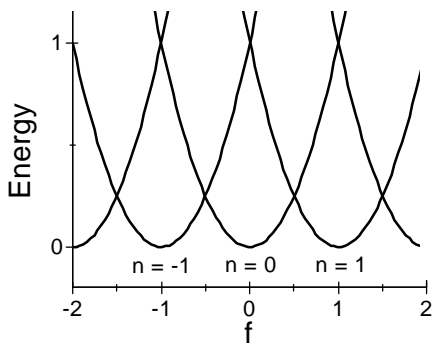

Figure 4.1: (a) Superconducting ring. $\gamma$ is the phase difference acquired around the ring. The flux in the ring is the externally applied flux plus the self induced flux. (b) Energy versus externally applied flux $f=\Phi_{\text {ext }} / \Phi_{0}$. The energy is normalized to $\Phi_{0}^{2} /\left(2 L_{K}(1+\beta)\right)$.

The flux $\Phi$ in the ring contains two contributions: the externally imposed flux $\Phi_{\text {ext }}=f \Phi_{0}$ and the flux $L_{G} I$ generated by the current, where $L_{G}$ is the geometrical inductance of the ring. The fluxoid quantization condition [8] and equation (4.1) yield:

$$
\gamma=-2 \pi \frac{\Phi}{\Phi_{0}}+2 \pi n=2 \pi \frac{n-f}{1+\beta}
$$


where $n$ is the integer fluxoid number and $\beta=L_{G} / L_{K}$ the ratio between geometrical and kinetic inductance. The current flowing in the ring

$$
I_{\text {ring }}=\frac{\Phi_{0}}{L_{K}(1+\beta)}(n-f)
$$

Changes in $n$ are only possible through phase slip processes, requiring the order parameter to go to zero in a region of the order of the coherence length. The energy required for a phase slip is [9]

$$
E_{B}=0.83 \frac{R_{q} s}{R_{n} \xi} k_{B} T_{c}=0.74 \frac{\hbar}{e^{2}} \frac{\Delta s}{R_{n} \xi}
$$

Here $R_{q}=h /\left(4 e^{2}\right)$ is the resistance quantum, $\xi$ the coherence length and $T_{c}$ the critical temperature of the material. As the resistance of the material is low, typically a few ohms per micrometer, the barrier is very high $>10,000 \mathrm{~K}$. A current flowing in the wire lowers the barrier. For small currents the decrease is given by

$$
E_{B}(I)=E_{B}-\frac{\Phi_{0}}{2} I
$$

The energy of the the ring has a parabolic dependence on the applied flux $f \Phi_{0}$ (Fig. $4.1(\mathrm{~b})$ ).

$$
E=\frac{1}{2} L_{K} I_{\text {ring }}^{2}+\frac{1}{2} L_{G} I_{\text {ring }}^{2}=\frac{\Phi_{0}^{2}}{2 L_{K}(1+\beta)}(f-n)^{2}
$$

If approximately one flux quantum is applied to the ring $(f \approx 1)$ while cooling down through the superconducting phase transition, the ring assumes the lowest energy state $n=1$. When the external field is removed at low temperatures, the new ground state $n=0$ cannot be reached and the ring remains frozen in the $n=1$ state. When a relatively weak superconducting circuit is attached to two contacts on the ring (Fig. 4.2 left), that circuit experiences a phase shift bias through two channels: the directly picked-up fraction of the total phase and the magnetic flux induced in the loop in the attached circuit. If the fluxoid conservation of such a circuit is considered [8]

$$
\oint \nabla \phi d \mathbf{s}=\frac{2 \pi}{\Phi_{0}}\left(\Phi_{\text {ext }}+\Phi_{\text {ind }}\right)
$$


the ring contributes on the left hand side of the equation with $\gamma a / s$, if the ring is homogeneous. Here $s$ is the total circumference of the ring and $a$ the enclosed section between the contacts. The integration path is indicated with the dashed line in figure 4.2. On the right hand side of equation (4.10) the contribution of the induced flux of the ring $\Phi_{\text {ind }}=\Phi_{\text {ind }}^{\prime}-M_{G} I_{\text {ring }}$ appears. $M_{G}$ is the mutual inductance between the ring and the circuit and $\Phi_{\text {ind }}^{\prime}$ the flux which is induced by other parts of the circuit. If one orders all the terms which depend on the magnetic field on the right-hand side of the equation and all the constants on the left, one gets

$$
\oint_{\text {circuit }} \nabla \phi d \mathbf{s}+2 \pi \alpha n=\frac{2 \pi}{\Phi_{0}} \Phi_{\text {ext }}+2 \pi \alpha f+\frac{2 \pi}{\Phi_{0}} \Phi_{\text {ind }}^{\prime}
$$

with

$$
\alpha=\frac{\frac{a}{s}+\frac{M_{G}}{L_{K}}}{1+\frac{L_{G}}{L_{K}}}=\frac{\frac{a}{s}+\frac{M_{G}}{L_{G}} \beta}{1+\beta}
$$

The action of the ring in a circuit is dual: The ring adds a fixed phase difference of $2 \pi \alpha n$ to the circuit. This part depends on the number of fluxoids $n$, which were frozen during the cool down. Additionally the ring contributes with $\alpha$ times of its area to the circuit area. For circuit considerations the ring can virtually be replaced with a fixed phase difference and a new integration path, as shown in figure 4.2 .

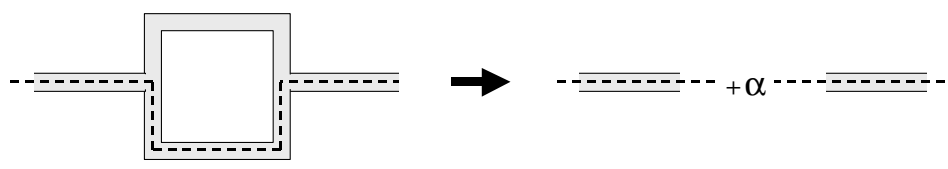

Figure 4.2: In a superconducting circuit the ring can virtually be replaced by a constant phase difference $2 \pi \alpha$ and a new integration path.

In the expression for $\alpha$ the contributions from the two channels can be observed. The ratio $a / s$ accounts for the phase difference between the contacts. It dominates if the kinetic inductance is large, i.e. $\beta \ll 1$. The ratio $M_{G} / L_{G}$ 
describes the contribution from the flux. It dominates when $\beta \gg 1$. In practice both limits can be realized, but intermediate values of $\beta$ are equally useful.

The flux applied to the ring during the cool down does not have to be exactly a flux quantum because $n$ is an integer. Any value between 0.5 and 1.5 flux quanta applied will result in $n=1$ state after the cool down.

\subsection{The $\pi$-SQUID and $\pi / 2$-SQUID}

The idea of the phase bias ring is applied to a SQUID. We fabricated three devices (Fig. 4.3) on a single substrate using standard aluminum shadow evaporation technique. The ring is evaporated in the same layer as the $\mathrm{Al} / \mathrm{Al}_{2} \mathrm{O}_{3} / \mathrm{Al}$ Josephson junctions, which makes the fabrication very simple. This is illustrated by the fact that the first fabrication attempt was successful. The first device (Fig. 4.3 (a)) is a standard SQUID and serves as a reference for the two other samples. The second device (Fig. 4.3 (b)) includes a ring in the SQUID, where one quarter of the ring is enclosed. We will refer to this device as the $\pi / 2$-SQUID. The third device (Fig. 4.3 (c)), called the $\pi$-SQUID, encloses half of the ring.

Measurements were performed in a dilution refrigerator at temperatures below $50 \mathrm{mK}$. We measured the switching current $I_{c}$ as a function of the applied magnetic field. All three devices show regular SQUID oscillations, with maxima and minima clearly shifted with respect to each other. Figure 4.4 shows the field dependence of the switching current for the reference and the $\pi$-SQUID. When the devices are cooled down without any flux applied to the ring, both devices show nearly the same oscillations with a maximum at zero magnetic field. The difference in the amplitude is due to a spread in the critical current of the junctions. After cooling down with one flux quantum applied to the ring, the oscillation of the $\pi$-SQUID is shifted by half a period with respect to the reference SQUID.

The $\pi / 2$-SQUID has an oscillation period that differs substantially from the two other devices. In order to compare the three samples we have plotted (Fig. 4.5) the normalized switching current as a function of normalized applied flux. The variable $n$ describes the number of flux quanta applied 

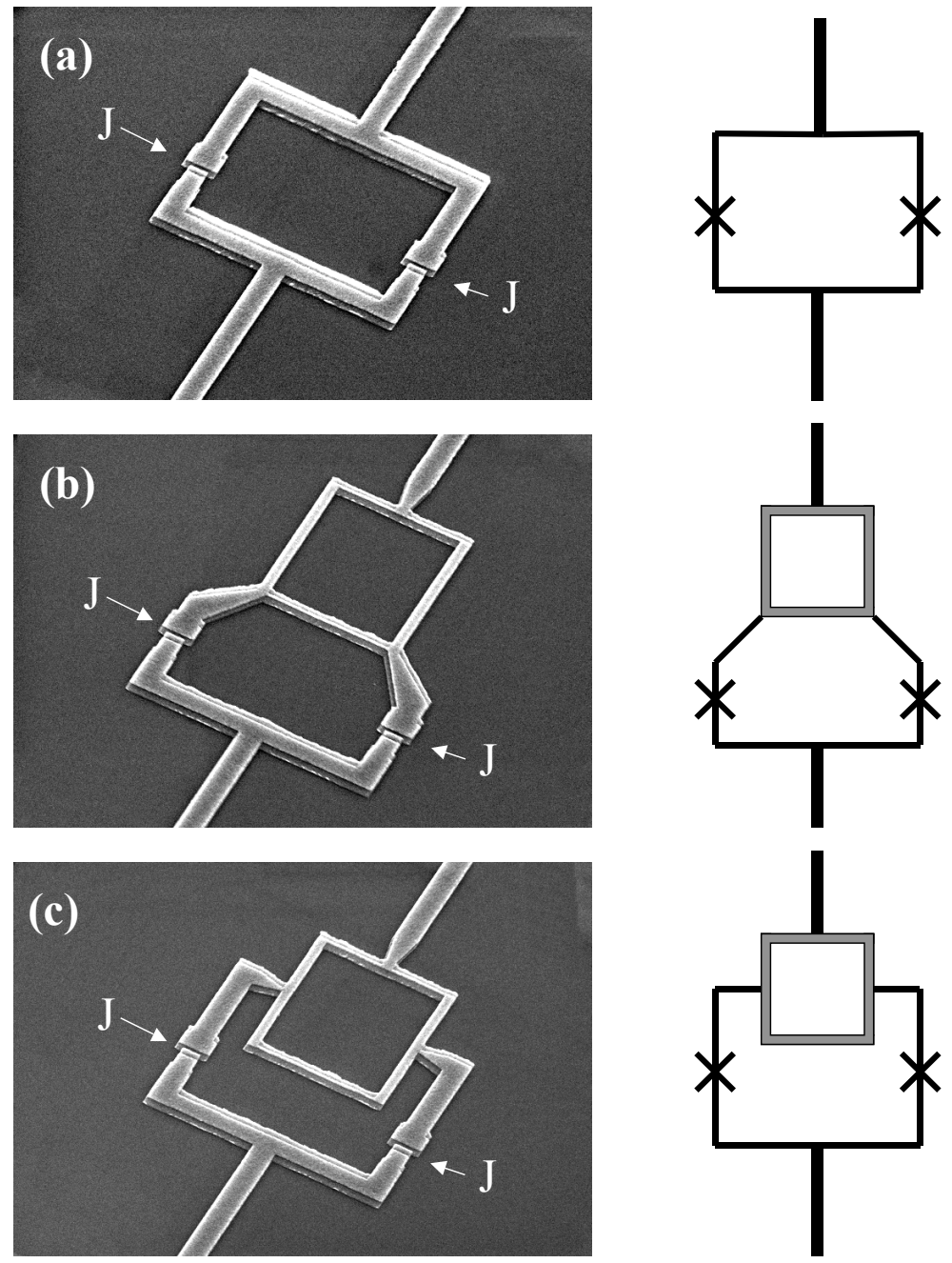

Figure 4.3: Left: Scanning electron images of the three devices. The Josephson junctions are indicated with J. Right: Schematic layout. The phase bias ring is shaded gray and the cross represents a Josephson junction. (a) shows the reference SQUID, (b) the $\pi / 2$-SQUID and (c) the $\pi$-SQUID. The ring has a diameter of $3 \mu \mathrm{m}$. 


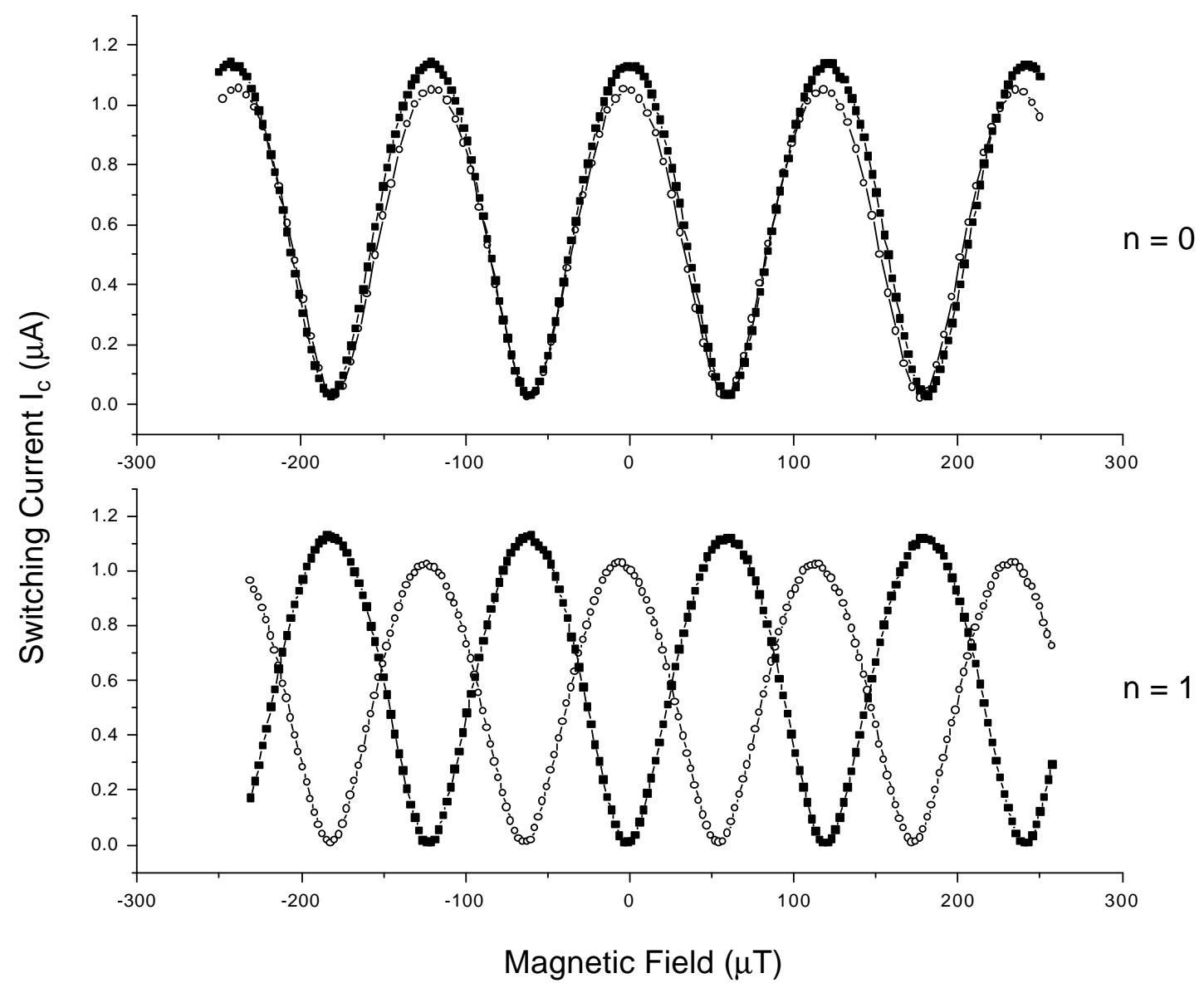

Figure 4.4: Switching current as a function of the applied magnetic field. The results for the reference SQUID are plotted with open symbols and for the $\pi$ SQUID with closed symbols. The variable $n$ counts the number of fluxoids frozen in the ring during cool down.

to the ring during cool down. Clearly observable is that the oscillations of the $\pi$-SQUID are shifted by $n$ times a half period. The oscillations of the $\pi / 2$-SQUID are shifted by $n$ times a quarter period.

For analysis, the critical current of the top measurements in figure 4.5 are Fourier analyzed. Results are given in table 4.1 .

Assuming that the current levels in the wire are much higher than the 



Figure 4.5: Normalized switching current as a function of the normalized applied flux. Top: Cooling down with one flux quantum applied to the ring. Middle: Cooling down with minus one flux quantum. Bottom: Cooling down with two flux quanta applied. 
Table 4.1: Results from a Fourier series fit of the measurements in Fig. (4.5).

\begin{tabular}{rrrr} 
Sample & Period & Maximum & Phase \\
\hline Reference SQUID & $118.2 \mu \mathrm{T}$ & $0.1 \mu \mathrm{T}$ & \\
$\pi / 2$-SQUID & $128.1 \mu \mathrm{T}$ & $32.5 \mu \mathrm{T}$ & $0.253 \cdot 2 \pi$ \\
$\pi$-SQUID & $120.5 \mu \mathrm{T}$ & $56.4 \mu \mathrm{T}$ & $0.467 \cdot 2 \pi$
\end{tabular}

critical current of the Josephson junctions $I_{0}$, i.e. $\Phi_{0} /\left(2 \pi L_{K}\right) \gg I_{0}$, equation (4.11) is applicable. For the critical current of a SQUID with a ring included the following expression results:

$$
I_{c}=2 I_{0}\left|\cos \left(\pi f_{\mathrm{SQUID}}+\pi\left(f_{\text {ring }}-n\right) \alpha\right)\right|
$$

Here $f_{\text {SQUID }} \Phi_{0}$ is the external flux in the SQUID loop and $f_{\text {ring }} \Phi_{0}$ the external flux in the ring. In expression 4.12 for $\alpha, M_{G}$ becomes the mutual inductance between the ring and the SQUID loop. The first part in the cosine argument gives the regular SQUID behavior. The second part is caused by the ring. The critical current pattern is shifted by $n \pi \alpha$. Additionally the ring contributes $\alpha$ times the area of the ring to the oscillation period. Furthermore in derivation of equation 4.13 the effects of the self inductance of the SQUID loop are neglected.

If the ring is small compared to the SQUID loop, the ratio between the mutual inductance $M_{G}$ and the self inductance $L_{G}$ of the ring is equal to the ratio between the enclosed section $a$ and the total circumference $s$. Then the $\alpha$ factor is just equal to these ratios $\alpha=a / s=M_{G} / L_{G}$.

In our experiment the ring is not small compared to the SQUID loop. The ratio $M_{G} / L_{G}$ for the $\pi / 2$-SQUID is 0.20 and for the $\pi$-SQUID 0.35 . This values are obtained with numerical methods [10].

To calculate the period and the phase shift with equation (4.13) a value for the kinetic inductance of the ring is needed. We have measured the resistance of a ring with the same dimensions as the ring in the $\pi$-SQUID and the $\pi / 2$-SQUID device. We measured a resistance value just above the superconducting transition of $R_{n}=18.0 \Omega$. With equation (4.4) one obtains a kinetic inductance of $L_{K}=20.2$ pico Henry and a ratio between self and kinetic inductance of $\beta=L_{G} / L_{K}=0.33$. The predicted values for the 
period and the phase of the $\pi$-SQUID and the $\pi / 2$-SQUID are shown in table 4.2. If the values are compared to the measured values in table 4.1 one observes a striking precision for the predicted oscillation period. Also the deviation from the predicted phase is maximally $1.5 \%$.

Table 4.2: Theoretical values for the $\pi$-SQUID and the $\pi / 2$-SQUID

\begin{tabular}{rrrr} 
Sample & Period & Maximum & Phase \\
\hline$\pi / 2$-SQUID & $128.2 \mu \mathrm{T}$ & $31.4 \mu \mathrm{T}$ & $0.244 \cdot 2 \pi$ \\
$\pi$-SQUID & $120.6 \mu \mathrm{T}$ & $55.6 \mu \mathrm{T}$ & $0.461 \cdot 2 \pi$
\end{tabular}

Given the precision of the fabrication this error lies well within expectations. Additionally the value for the resistance $R_{n}$ is obtained with a sample on a different substrate and a different fabrication run than the three SQUID devices.

The reason why the intended phase shifts of $\pi / 2$ and $\pi$ are not reached, is that the ratios of the mutual and the self inductance of the ring $M_{G} / L_{G}$ deviate from $1 / 2$ resp. $1 / 4$. To improve that, one would need to make the area of the ring much smaller compared to the area of the SQUID. Making the area of the SQUID larger has the disadvantage that more flux noise is picked up by the SQUID. Making the ring smaller has the disadvantage that the current flowing in the ring becomes higher. Hence the barrier for phase slips (4.8) is lowered. In practice a good trade-off has to be found.

Instead of warming up and cooling down to freeze a different number of fluxoids in the ring, one can also apply a high current to the device. The junctions are in the voltage stage and dissipate energy. This heat warms up the rest of the circuit including the ring and brings it in the normal state. After applying the specific flux to the ring the current is switched off and the device becomes superconducting again.

\subsection{Applications}

Phase-bias rings can be applied for flux qubits [3], which need a shift of $\pi$ to reach the degeneracy point where superposition states occur. Furthermore the SQUID that measures the qubit has to be biased at a sensitive point, 
which is at about $\pi / 2$. The ring bias is expected to have much lower flux noise than an external magnetic field. Figure 4.6 shows a simple version of a gradiometer qubit where both the qubit and the measuring SQUID are biased by the same ring. The qubit with the three Josephson junctions is placed at the inside of the bias ring. This has the advantage that the qubit is insensitive to external flux noise in first order. The flux bias line is used to apply a small asymmetric flux bias which is needed to control the energy levels of the qubit. Furthermore it can be used to apply the flux quantum to the bias ring during the cool down.

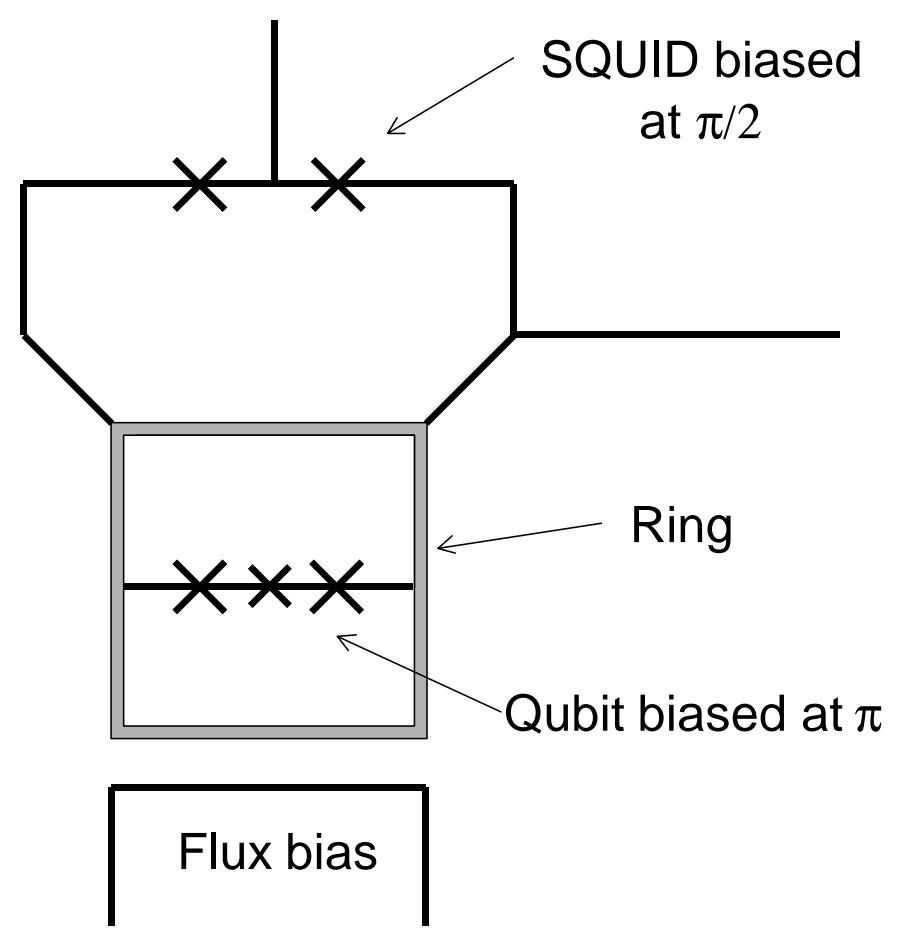

Figure 4.6: Simplified flux qubit scheme. With a single ring the qubit is biased at $\pi$ and the read-out SQUID at $\pi / 2$.

A similar scheme for a SQUID with reduced flux noise is shown in figure 4.7. Two Josephson junctions forming a SQUID are placed in a bias ring. The SQUID occupies a quarter of the ring area. The gradiometer action can be understood with equation (4.13): The ratio between the enclosed 
section and the total circumference is $a / s=1 / 4$. Also the ratio between mutual inductance and self inductance is $M_{G} / L_{G}=1 / 4$. Therefore $\alpha=1 / 4$ independently of the value of the kinetic inductance of the ring. The flux in the ring $f_{\text {ring }} \Phi_{0}$ is four times bigger than the flux in the SQUID $f_{\text {SQUID }} \Phi_{0}$. Because the SQUID is located inside the ring $f_{\mathrm{SQUID}} \Phi_{0}$ gets an extra negative sign in equation (4.13). Therefore the contributions of the external field cancel and the switching current $I_{c}$ is independent of the magnetic field. In order to couple a signal in the SQUID an asymmetric flux bias can be applied with a flux bias line next to the ring. Like in the previous example this line can also be used to apply the flux quantum to the ring during cool down. To connect the SQUID the bias ring has to be crossed by a contact line. This crossing is very hard to realize with the technique described in the introduction. But with multi layer techniques developed for rapid single flux quantum logic (RSFQ) circuits [11] such a crossing can easily be fabricated.

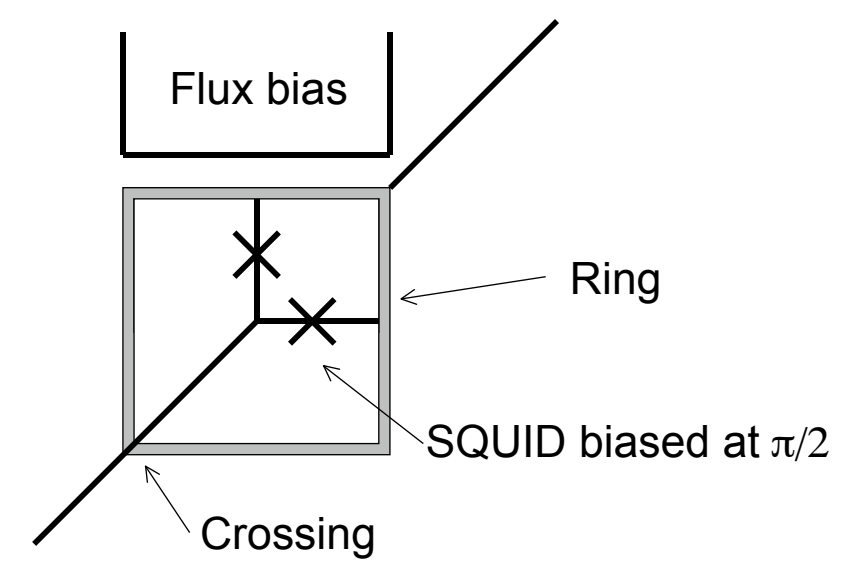

Figure 4.7: Gradiometer SQUID scheme. The SQUID is placed at the inside of a bias ring, occupies a quarter of the area of the ring and is biased at $\pi / 2$ by the ring. The flux bias line allows to couple a signal into the SQUID and applies a flux quantum to the ring during cooling down.

Another application of the phase bias ring is the complementary Josephson junction logic (CJJ) introduced by Terzioglu and Beasley [5]. This logic 
family is based on the analogy to complementary metal-oxide-semiconductor (CMOS). The logic levels are not high or low voltage like in CMOS, but high or low superconducting current. It utilizes two types of devices: a conventional device, which has a finite critical current at zero input and zero critical current at finite input. A standard SQUID meets these requirements (Fig. 4.8 right bottom). Secondly a complementary device with zero critical current at zero input and finite critical current at finite bias. The $\pi$-SQUID presented in the previous chapter is exactly such a device (Fig. 4.8 right top). Figure 4.8 shows an inverter based on this complementary Josephson junction logic.
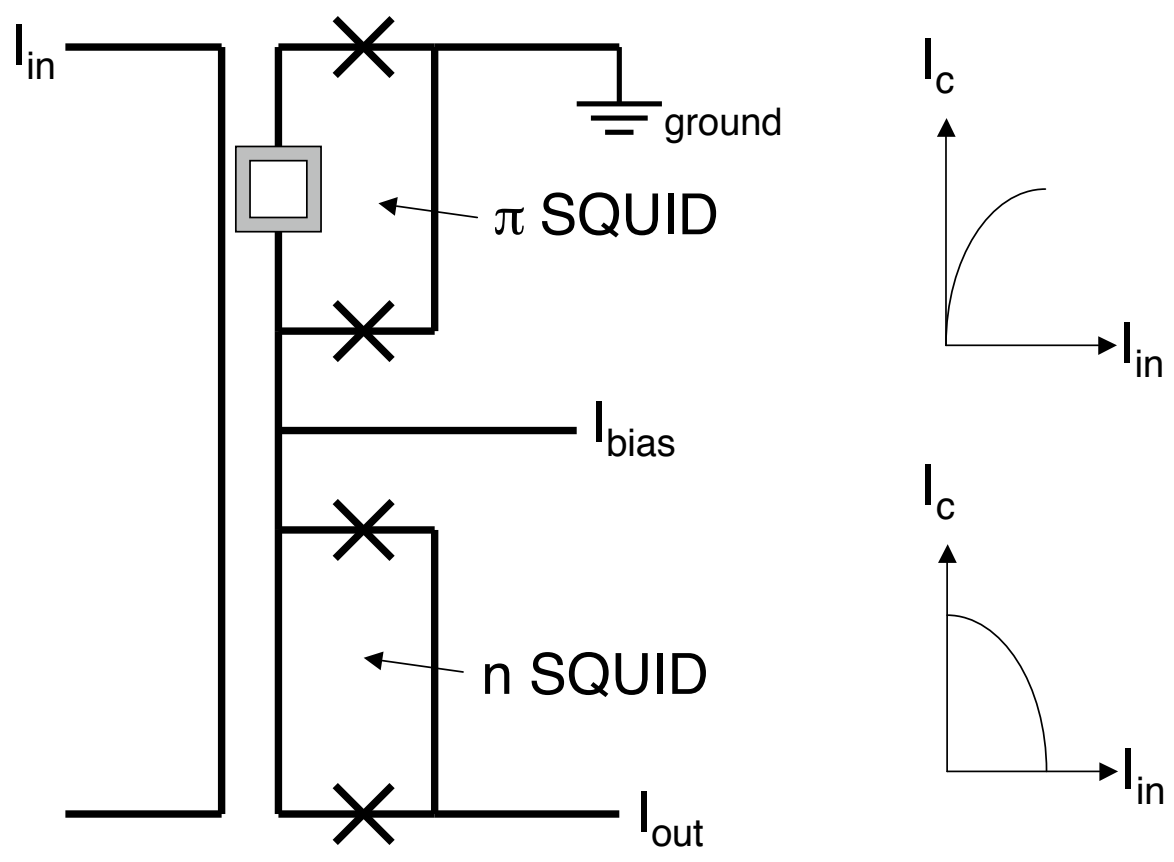

Figure 4.8: Scheme for a superconducting inverter based on complementary Josephson logic.

The input is just a superconducting wire which couples an equal amounts of flux in the SQUID and the $\pi$-SQUID. Both SQUIDs are connected to the bias current. The other contact of the $\pi$-SQUID is connected to ground. The second contact of the SQUID forms the output of the circuit. If no 
current is applied to the input, the logical zero, the critical current of the $\pi$-SQUID is zero and the supercurrent will flow via the normal SQUID to the output. This forms a logical one at the output. A logical one at the input is represented by a current applied to the input of the circuit, such that half a flux quantum is induced in the $\pi$-SQUID and the normal SQUID. Now the $\pi$-SQUID has a high critical current and the critical current of the normal SQUID is zero. The bias current flows via the $\pi$-SQUID to ground and a logical zero is present at the output.

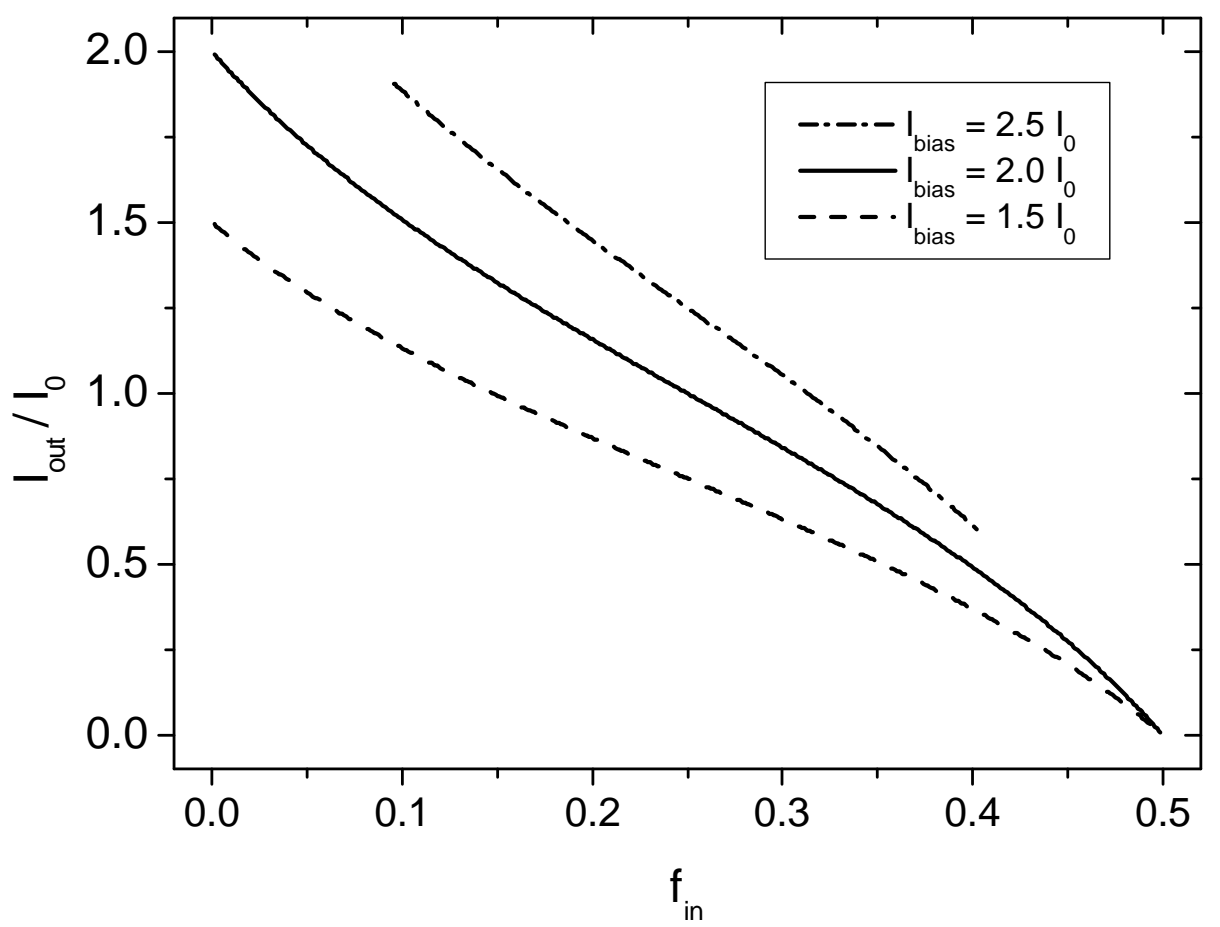

Figure 4.9: Inverter characteristic: The output current $I_{\text {out }}$ as a function of the flux produced by the input current $f_{\text {in }}$ for different values of the bias current $I_{\text {bias }}$.

Figure 4.9 shows the output current as a function of the flux $f_{\text {in }} \Phi_{0}$ induced by the input current. If the bias current is bigger than twice the critical current of a single junction the circuit is forced into a voltage state. This explains the missing solutions for a bias of $I_{\text {bias }}=2.5 I_{0}$ below $0.1 \Phi_{0}$ and above $0.4 \Phi_{0}$. 
In order to cascade such logic circuits the inverter has to provide current gain. This means that the maximum output current has to produce at least half a flux quantum in the following device. The gain for the inverter circuit is given by

$$
G=4 \frac{I_{0} M_{G}}{\Phi_{0}}
$$

where $I_{0}$ is the critical current of a single Josephson junction and $M_{G}$ the mutual inductance between the input wire and the SQUID or the $\pi$-SQUID. With the shadow evaporation technique described in the introduction critical currents of few micro Ampères and mutual inductances of few pico Henry can maximally be achieved. Therefore the requirement of $G \geq 1$ can not be met with shadow evaporation technique. But with fabrication technologies developed for RSFQ [11] a gain of greater than one can be fabricated. The input line can be used during cooling down to produce the flux quantum needed in the bias ring.

Besides a simple inverter also more complicated circuits can be made with complementary Josephson junction logics. Figure 4.10 shows the scheme for a NOR gate. There is only an output current if both input currents are zero. Similar to CMOS logic this logic family does not consume power in the the non-switching state, because everything is superconducting. In contrast shunt resistors are needed for RSFQ logics. This absence of dissipation makes it also a very good candidate for control logics of quantum circuits. 


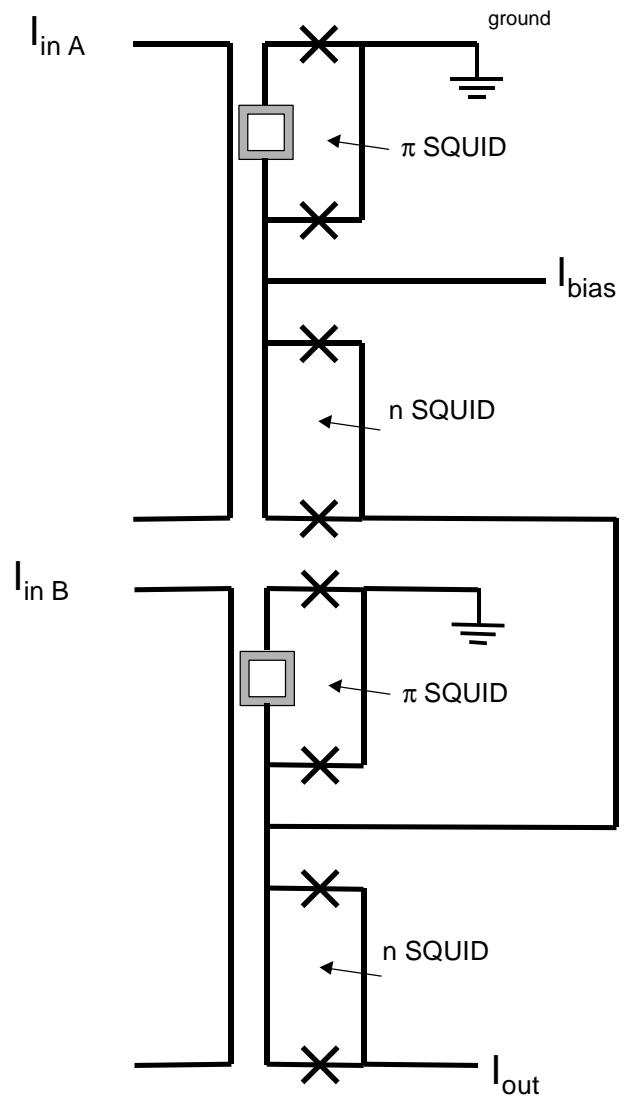

Figure 4.10: A NOR Gate realized with complementary Josephson junction logics. 


\section{References}

[1] M. Tinkham, "Introduction to Superconductivity," McGraw-Hill, New York, (1996).

[2] L. B. Ioffe, V. B. Geshkenbein, M. V. Fiegel'man, A. L. Fauchere, and G. Blatter, "Environmentally decoupled sds -wave Josephson junctions for quantum computing," Nature 398, 670 (1999).

[3] J. E. Mooij, T. P. Orlando, L. Levitov, L. Tian, C. H. van der Wal, and S. Lloyd, "A Superconducting Persistent Current Qubit," Science 285, 1036 (1999).

[4] G. Blatter, V. B. Geshkenbein, L. B. Ioffe, "Design aspects of superconductingphase quantum bits," Phys. Rev. B 63, 174511 (2001).

[5] E. Terzioglu, M. R. Beasley, IEEE Trans. Appl. Supercond. 8, 48 (1998).

E. Terzioglu, D. Gupta and M. R. Beasley, IEEE Trans. Appl. Supercond. 7, 3642 (1997).

[6] E. Il'ichev, M. Grajcar, R. Hlubina, R. P. J. IJsselsteijn, H. E. Hoenig, H.-G. Meyer, A. Golubov, M. H. S. Amin, A. M. Zagoskin, A. N. Omelyanchouk, and M. Yu. Kupriyanov, "Degenerate Ground State in a Mesoscopic $\mathrm{YBa}_{2} \mathrm{Cu}_{3} \mathrm{O}_{7} \mathrm{x}$ Grain Boundary Josephson Junction," Phys. Rev. Lett. 86, 5369 (2001).

[7] V. V. Ryazanov, V. A. Oboznov, A. Yu. Rusanov, A. V. Veretennikov, A. A. Golubov and J. Aarts, "Coupling of Two Superconductors through a Ferromagnet: Evidence for a pi Junction," Phys. Rev. Lett. 86, 2427 (2001).

[8] T. P. Orlando, K. A. Delin, "Foundations of Applied Superconductivity," Addison-Wesley Publishing Company (1991).

[9] C.N. Lau, N. Markovic, M. Bockrath, A. Bezryadin and M. Tinkham, "Quantum Phase Slips in Superconducting Nanowires," Phys. Rev. Lett. 87, 217003 (2001).

[10] M. Kamon, M. J. Tsuk, and J. White. "FastHenry: A multipole-accelerated 3-D inductance extraction program." In Design Automation Conference, pages 678-683 (1993). 
[11] Hypres, Inc. http://www.hypres.com Lincoln Laboratory http://www.1l.mit.edu 


\title{
Chapter 5
}

\section{Coupling of qubits}

\author{
J. B. Majer, F. G. Paauw, J. E. Mooij
}

A. C. J. ter Haar, C. J. P. M. Harmans, L. S. Levitov

We study the coupling of persistent-current qubits. We show that two qubits can be coupled inductively. The interaction is of Ising type $\sigma_{1}^{z} \sigma_{2}^{z}$. The energy spectrum of two coupled qubits is calculated in the ideal identical case as well as for two non identical qubits. We designed and fabricated a sample with two coupled qubits and a SQUID that measures the two qubits. Measurements with applied microwaves show transitions between two qubit states.

Parts of this chapter have been published in cond-mat/0108266. 


\subsection{Introduction}

Quantum computers are machines that store their information in quantum variables, so-called quantum bits. This information is processed by making the quantum bits interact with each other in a way that preserves quantum coherence $[1,2,3]$. Many algorithms have been invented where it has been shown that quantum computers would be substantially faster than classical computers. The most prominent are Shor's factoring algorithm [4] and Grover's algorithm to search unstructured databases [5].

Experimentally, quantum bits have been realized in a variety of systems, like cavity quantum electrodynamics [6], ion traps [7] and nuclear spins [8]. Recently Shor's factoring algorithm was demonstrated with the latter system [9]. These systems have all very long decoherence times. However, it will be very difficult to produce quantum computers with a larger number of quantum bits.

With quantum computers fabricated by modern lithography one hopes to circumvent this limitation. Several schemes have been proposed based on individual donor atoms in silicon [10], spin states in quantum dots [11] and circuits with small Josephson junctions. At the moment, quantum bits based on Josephson junctions are most advanced. One can distinguish two regimes. On the one hand the charging regime, where the states of the quantum bit are formed by different amounts of charge on an island [14, 15]. For such charge quantum bits, coherent oscillations have been demonstrated [16]. The opposite regime is the phase regime. Here the basic states are formed by different superconducting phase configurations [12, 13]. Superposition of states has been demonstrated [17, 18]. Recently Rabi oscillations of a persistent-current qubit have been measured [19, 20]. A system which takes advantage of both regimes also showed coherent oscillations [21] .

The next step to build a solid state quantum gate is to let the qubits interact with each other. In this chapter we treat the static coupling of two persistent-current qubits. We mainly focus on the inductive coupling of the qubits. First we calculate the energy spectrum of two identical qubits. Then we treat the more realistic case of two coupled qubits with small asymmetries. In the following section we show the design and the fabrication of 
a sample with two coupled qubits. Furthermore the measurement SQUID and its environment is described. At the end we show first results, which demonstrate two qubit level transitions.

\subsection{Persistent-current qubit as quasi-spin}

A persistent-current qubit consists of a superconducting ring which is interrupted by three small Josephson junctions (Fig. 5.2 left). One of the three junctions is slightly smaller (by a factor $\alpha<1$ ) than the other two. Two energy scales determine the behavior of the Josephson junctions and therefore of the persistent-current qubit. The first energy is the Josephson coupling energy given by $E_{J}=I_{0} \Phi_{0} /(2 \pi)$, where $I_{0}$ is the critical current of the junction and $\Phi_{0}=h /(2 e)$ the superconducting flux quantum. The second energy is the Coulomb energy for a single electron $E_{c}=e^{2} / 2 C$. Here $e$ is the charge of an electron and $C$ the capacitance of the junction. The state of the qubit can be expressed by the superconducting phase and the number of Cooper pairs on each node $[12,13]$. Phase and charge are quantum-mechanical conjugate variables [22]. When the two energies $E_{J}$ and $E_{c}$ are within a few orders of magnitude of each other, the eigenstates of the system are formed by superpositions of phase or charge states. In reference [23] one can find a numerical program that calculates eigenfunctions and eigenenergies of the persitent-current qubit. Furthermore a rigorous derivation of the Hamiltonian of the system is included. Figure 5.1 shows the eigenenergies as a function of the externally applied flux $f \Phi_{0}$. The result is obtained with a ratio of the Josephson and the charging energy of $E_{J} / E_{c}=60$. The size of the smaller junction is $80 \%$ of the two other junctions $(\alpha=0.8)$.

Between $\mathrm{f}=0.48$ and $\mathrm{f}=0.52$ flux quanta applied to the qubit, the classical eigenstates are states with clockwise and anti-clockwise circulating currents. The clockwise circulating current state $|\downarrow\rangle$ produces a magnetic field which points into the plane of the qubit (Figure 5.2 middle). Similarly the anticlockwise state produces a field which points out of the plane (Figure 5.2 right). In a very small region at around half a flux quantum, a superposition of the two states exists, which leads to a rounding of the levels and an energy gap at half a flux quantum. The system can then be approximated by a 


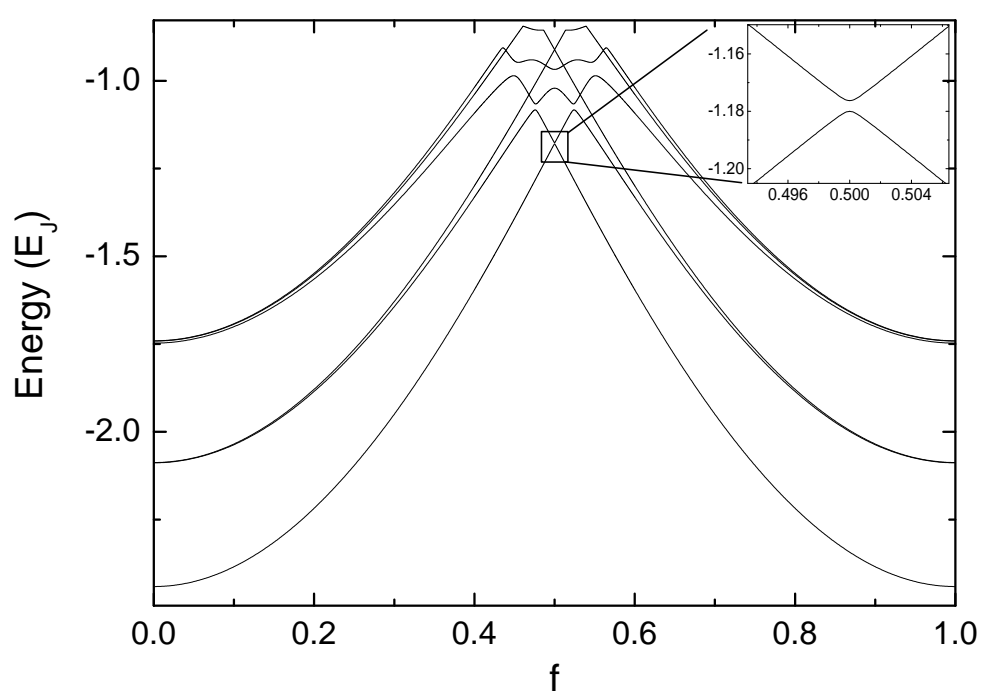

Figure 5.1: Energy levels of a persistent-current qubit. Plot of the qubit energy in units of the Josephson energy $E_{J}$ vs the external magnetic flux $f \Phi_{0}$. In a region at about half a flux quantum the two lowest levels are states with clockwise and anti-clockwise circulating currents. At half a flux quantum a superposition of these two current states exists. The parameters for the energy levels plotted are $E_{J} / E_{c}=60$ and $\alpha=0.8$.

Hamiltonian which is similar to the Hamiltonian of a spin in a magnetic field (quasi spin):

$$
\mathcal{H}_{\text {qubit }}=h \sigma^{z}+t \sigma^{x}
$$

Here $h$ is a parameter which depends on the flux $\Phi$ in the qubit $h=I_{p}(\Phi-$ $\left.\Phi_{0} / 2\right)$, where $I_{p}$ is the circulating current. The tunneling element $t$ depends exponentially on the ratio $E_{J} / E_{c}[13]$, and is half the energy gap. Analogous to the magnetic moment of a real spin one can understand the magnetic moment produced by the circulating current.

However, it is important to recognize where the limitations of the two level approximation are. One can identify two important limits. First where the energy difference between the first excited state and the ground state $\left(\Delta_{10}=E_{1}-E_{0}\right)$ becomes smaller than the difference between the second and the first excited state $\left(\Delta_{21}=E_{2}-E_{1}\right)$. This limit is important when 


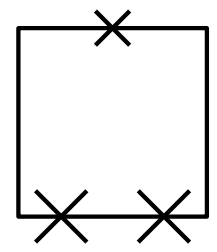

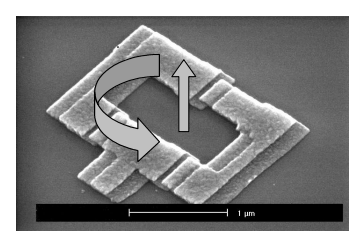

spin up

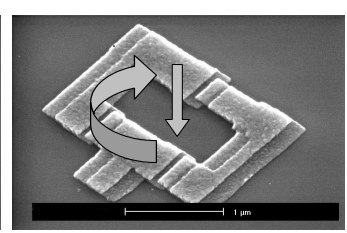

spin down

Figure 5.2: Left: Scheme of the persistent-current qubit with the three Josephson junctions indicated by a cross. Middle and right: The circulating current states. The anti-clockwise state $|\uparrow\rangle$ (middle) produces a magnetic field which points out of the plane and the clockwise circulating state $|\downarrow\rangle$ (right) which points into the plane.

one probes the energy differences between the states, e.g. by spectroscopy. The second limit is the position where the first excited state has a maximum. This limit is important for ground state considerations. In a coupled system, the ground state of the system can be formed by combinations of the ground and the first excited state of a single system. Therefore it is important to know where the energy difference does not anymore increase linearly with the magnetic field. This happens where the first excited state reaches its maximum. Figure 5.3 shows these two limits as a function of the energy ratio $E_{J} / E_{c}$.

\subsection{Qubit coupling mechanisms}

\subsubsection{Inductive coupling}

A very simple way to couple two persistent-current qubits is to put them very close to each other. The circulating current of one qubit produces an extra flux in the neighboring qubit. Depending on the direction of the circulating current, the flux either adds or subtracts to the externally applied flux and therefore changes the energy levels. The Hamiltonian describing two coupled systems is

$$
\mathcal{H}=\mathcal{H}_{\text {qubit } 1}+\mathcal{H}_{\text {qubit } 2}=h_{1}^{\text {tot }} \sigma_{1}^{z}+t_{1} \sigma_{1}^{x}+h_{2}^{\text {tot }} \sigma_{2}^{z}+t_{2} \sigma_{2}^{x}
$$



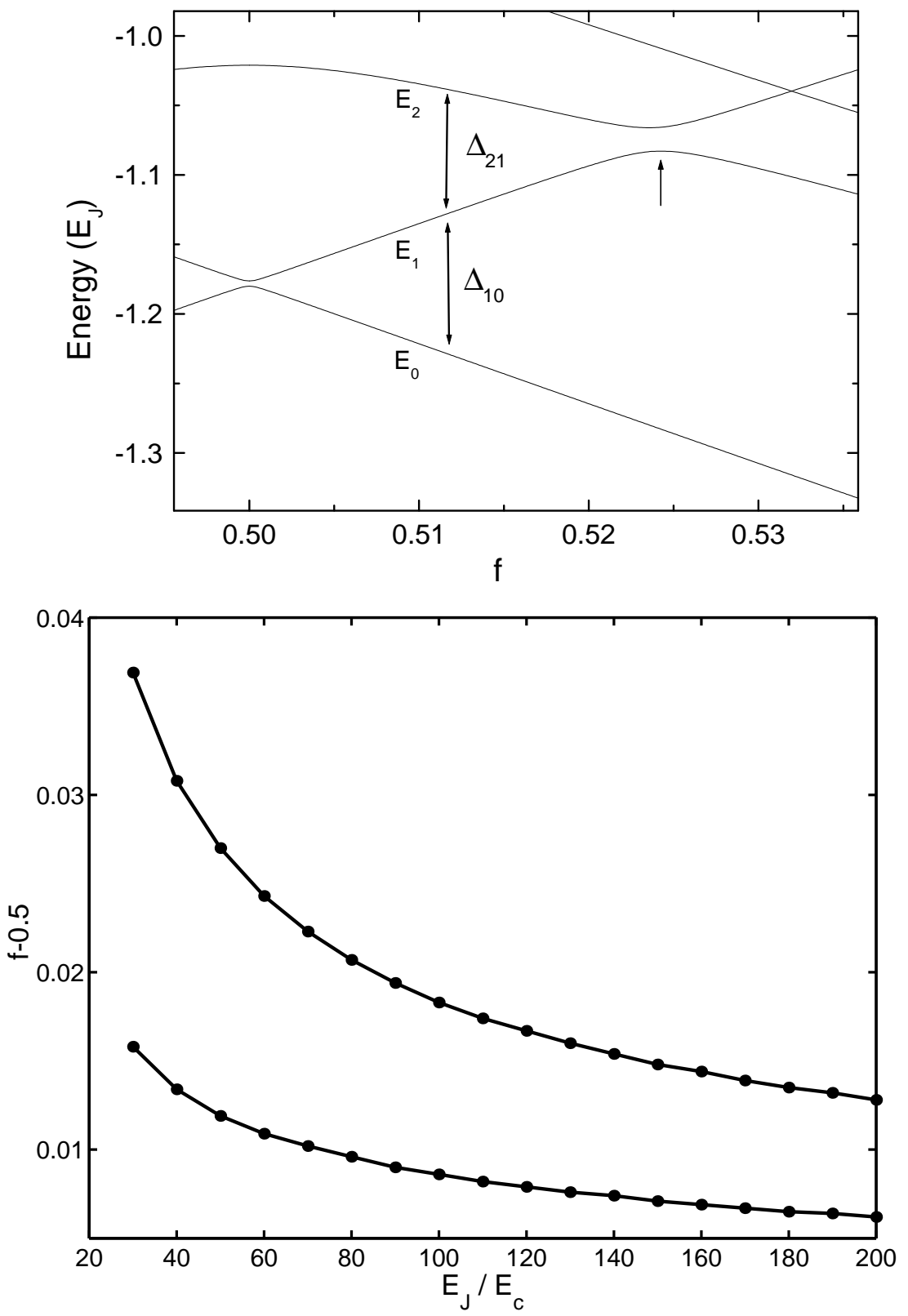

Figure 5.3: Limitations of the two-level approximation. First limit is where the energy difference $\Delta_{21}=E_{2}-E_{1}$ (between second excited and first excited state) becomes smaller than $\Delta_{10}=E_{1}-E_{0}$ (difference between first excited state and ground state). Second limit is where the first excited state has a maximum. Below: Dependence of the two limits on the energy ratio $E_{J} / E_{c}$ with a fixed $\alpha=0.8$. 
For $h_{1}^{\text {tot }}$ one has to consider three contributions. The externally applied magnetic field $\Phi_{1}^{\text {ext }}$, the field induced by the neighboring qubit $\Phi_{1}^{\text {induced }}$ and the field produced by the qubit itself $\Phi_{1}^{\text {self }}$.

$$
\begin{aligned}
h_{1}^{\text {tot }} & =I_{p, 1}\left(\Phi_{1}^{\text {tot }}-\frac{1}{2} \Phi_{0}\right) \\
& =I_{p, 1}\left(\Phi_{1}^{\text {ext }}+\Phi_{1}^{\text {self }}+\Phi_{1}^{\text {induced }}-\frac{1}{2} \Phi_{0}\right)
\end{aligned}
$$

The two induced fields depend on the states of the two qubits. The self induced flux is the current $I_{1}$ in the qubit times the selfinductance $L_{1}$

$$
\Phi_{1}^{\text {self }}=L_{1} I_{1}=-L_{1} I_{p, 1} \sigma_{1}^{z}
$$

Similarly, the field induced by the neighboring qubit depends on the mutual inductance between the two qubits.

$$
\Phi_{1}^{\text {induced }}=M I_{2}=M I_{p, 2} \sigma_{2}^{z}
$$

The same expressions hold for the second qubit. For the external magnetic field $h=I_{p, 1}\left(\Phi_{1}^{\text {ext }}-\Phi_{0} / 2\right)$ one gets a constant coupling term $\sigma_{1}^{z} \sigma_{2}^{z}$ with magnitude $j$. The Hamiltonian becomes

$$
\mathcal{H}=\mathcal{H}_{\text {qubit } 1}+\mathcal{H}_{\text {qubit } 2}=h_{1} \sigma_{1}^{z}+t_{1} \sigma_{1}^{x}+h_{2} \sigma_{2}^{z}+t_{2} \sigma_{2}^{x}+\underbrace{2 M I_{p, 1} I_{p, 2}}_{=j} \sigma_{1}^{z} \sigma_{2}^{z}
$$

Here the terms due to the self inductance are neglected. They are of the form $\sigma_{z}^{2}=\mathbf{1}$ and just add a constant energy term.

Experimentally it is difficult to reach a significant coupling by just putting the qubits next to each other. To increase the coupling strength the two qubits share one lead as shown in figure 5.4. This increases the mutual inductance but does not change the behavior of the single qubit.

If one assumes the two qubits to be equal, the Hamiltonian in the basis of the two qubit states $|\downarrow \downarrow\rangle,|\downarrow \uparrow\rangle,|\uparrow \downarrow\rangle,|\uparrow \uparrow\rangle$ takes the following form

$$
\mathcal{H}=\left(\begin{array}{cccc}
-2 h+j & t & t & 0 \\
t & -j & 0 & t \\
t & 0 & -j & t \\
0 & t & t & 2 h+j
\end{array}\right)
$$



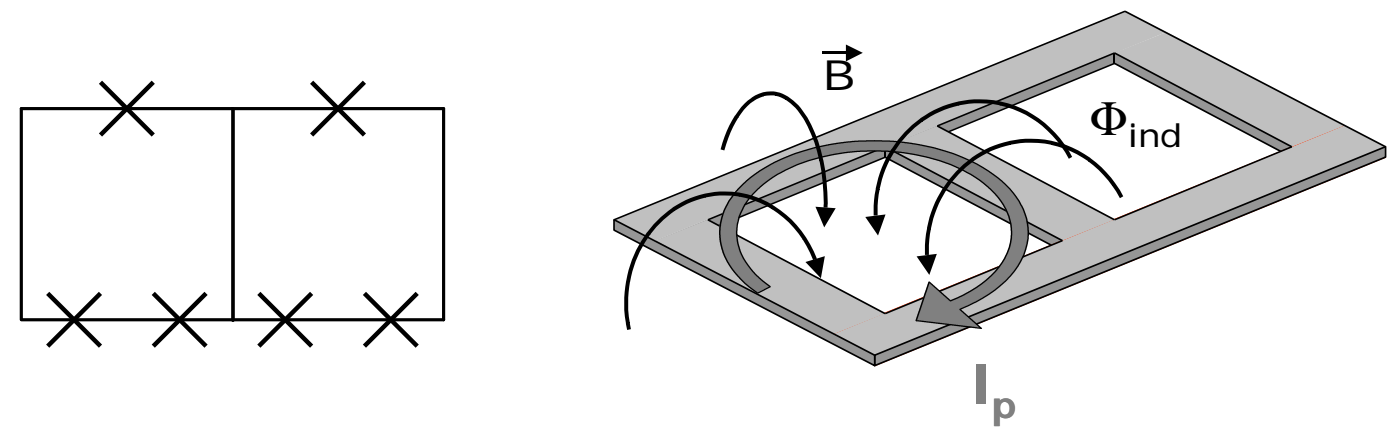

Figure 5.4: Inductive coupling two qubits by sharing one lead. The currents in one qubit produce an extra magnetic field in the neighboring qubit which changes the energy levels in the second qubit.

Figure 5.5 top shows the energy levels vs. the external magnetic field. Far away from half a flux quantum, $h \gg t$, the energy states assume the classical form and the first and the second excited state are energetically degenerate

$$
\begin{array}{rlrl}
h \gg t & r l & \\
E_{0} & =-2 h+j & \left|\psi_{0}\right\rangle & =|\downarrow \downarrow\rangle \\
E_{1} & =-j & \left|\psi_{1}\right\rangle & =|\downarrow \uparrow\rangle-|\uparrow \downarrow\rangle \\
E_{2} & =-j & \left|\psi_{2}\right\rangle & =|\downarrow \uparrow\rangle+|\uparrow \downarrow\rangle \\
E_{3} & =2 h+j & \left|\psi_{3}\right\rangle & =|\uparrow \uparrow\rangle
\end{array}
$$

At half a flux quantum, $h=0$, the states are superpositions of the original single qubit states. The degeneracy of the second and the first excited state is lifted, yielding

$$
\begin{aligned}
h=0 & & & \\
E_{0} & =-\sqrt{j^{2}+4 t^{2}} & & \left|\psi_{0}\right\rangle=|\uparrow \uparrow\rangle+|\downarrow \downarrow\rangle-\frac{j+\sqrt{j^{2}+4 t^{2}}}{2 t}(|\uparrow \downarrow\rangle+|\downarrow \uparrow\rangle) \\
E_{1} & =-j & & \left|\psi_{1}\right\rangle=|\downarrow \uparrow\rangle-|\uparrow \downarrow\rangle \\
E_{2} & =j & & \left|\psi_{1}\right\rangle=|\uparrow \uparrow\rangle-|\downarrow \downarrow\rangle \\
E_{3} & =\sqrt{j^{2}+4 t^{2}} & & \left|\psi_{3}\right\rangle=|\uparrow \uparrow\rangle+|\downarrow \downarrow\rangle-\frac{j-\sqrt{j^{2}+4 t^{2}}}{2 t}(|\uparrow \downarrow\rangle+|\downarrow \uparrow\rangle)
\end{aligned}
$$

One observes that the energy of the first excited state does not depend on the externally applied flux. This state is the singlet state formed by 

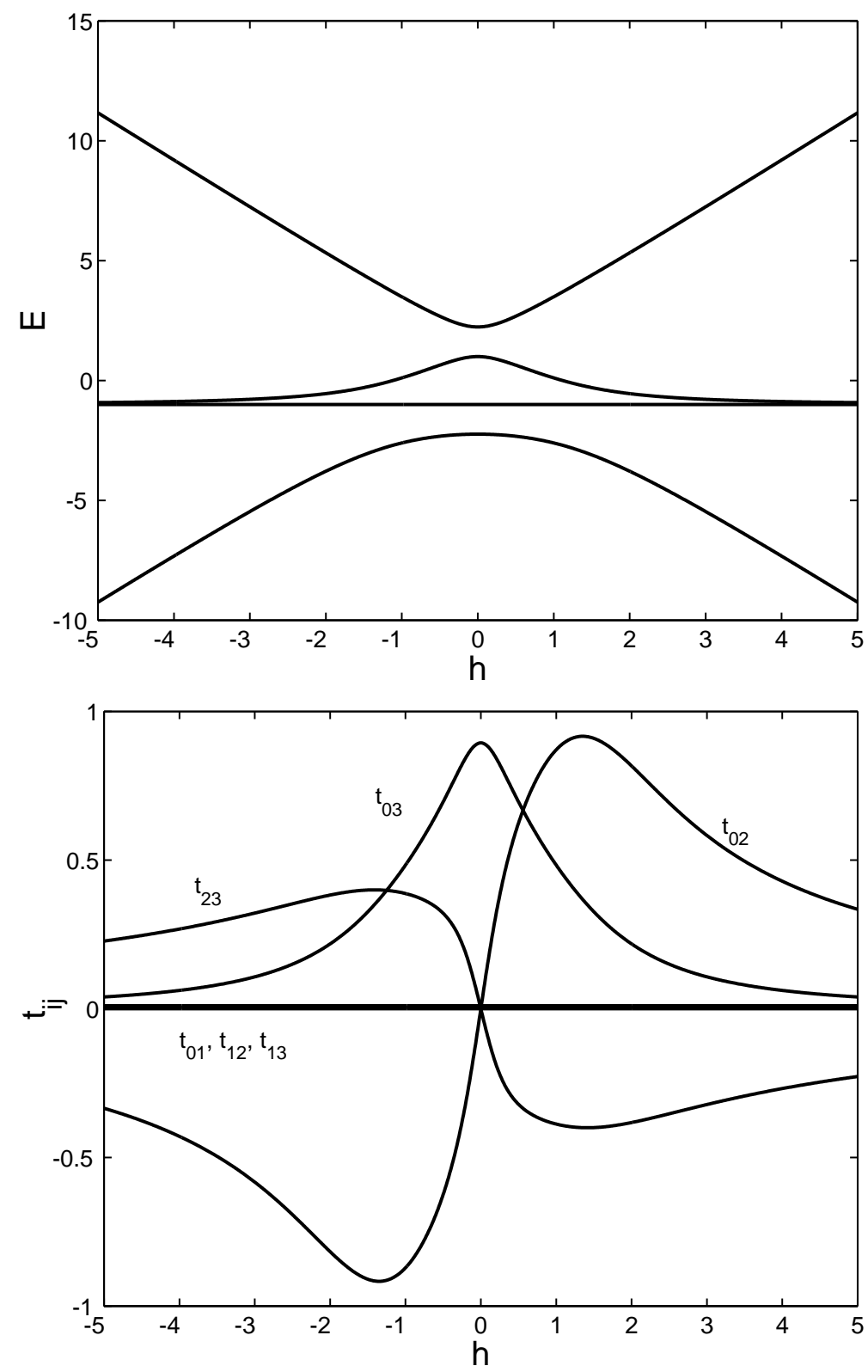

Figure 5.5: Above: Energy for two equal coupled qubits. Below: Transition elements between the energy states. The first excited state is the singlet state and is independent of the external flux. Because of the symmetry of the singlet state all transition elements including that state are zero. Here the coupling is $j=1$ and $t=1$. 
the anti-symmetric superposition $|\downarrow \uparrow\rangle-|\uparrow \downarrow\rangle$. Due to the symmetry of the Hamiltonian this singlet state is independent of the external flux.

Figure 5.5 (bottom) shows the transition elements $t_{i j}=\left\langle\psi_{i}\left|\sigma_{z, 1}+\sigma_{z, 2}\right| \psi_{j}\right\rangle$ as a function of the external magnetic field $h$. This form of the transition element follows if one assumes that the transitions are induced by an external homogeneous rf-field ( $\sigma_{z}$ excitation). One observes that any transition involving the singlet state (first excited state) is forbidden. The reason is that a symmetric operator $\sigma_{z, 1}+\sigma_{z, 2}$ can not induce transitions between the anti-symmetric singlet state and the symmetric triplet states. Furthermore the transition element for the transition between ground state and highest excited state $t_{03}$ decreases much faster away from half a flux quantum than the transition element $t_{02}$. This means that it is much harder to induce the transition $|\downarrow \downarrow\rangle \rightarrow|\uparrow \uparrow\rangle$ than the transition $|\downarrow \downarrow\rangle \rightarrow|\downarrow \uparrow\rangle+|\uparrow \downarrow\rangle$.

For a realistic sample one can never assume the two qubits to be perfectly equal. Three sources of asymmetry can be identified: The junctions of the two qubits can be different. This leads to different persistent currents $I_{p, 1}, I_{p, 2}$ in the two qubits as well as different tunneling elements $t_{1}, t_{2}$. We define the average persistent current $I_{p}$ and the relative difference $\gamma$ :

$$
I_{p}:=\frac{I_{p, 1}+I_{p, 2}}{2} \quad \gamma:=\frac{I_{p, 1}-I_{p, 2}}{I_{p}}
$$

Furthermore the areas enclosed by the two qubits can be different. Analogously, we define the average area $S$ and the relative difference $\sigma$

$$
S:=\frac{S_{1}+S_{2}}{2} \quad \sigma:=\frac{S_{1}-S_{2}}{S}
$$

Finally the magnetic fields penetrating the qubits can be different. This can be achieved by introducing experimentally a controlled asymmetry. We define the average magnetic field $B$ and the difference between the two fields $B_{\text {asymm: }}$

$$
B:=\frac{B_{1}+B_{2}}{2} \quad B_{\text {asymm }}:=B_{1}-B_{2}
$$

If the field is not homogeneous in the qubit, the fields $B_{1}$ and $B_{2}$ can be defined as the average fields $B_{1}=\Phi_{1}^{\text {ext }} / S_{1}$ and $B_{2}=\Phi_{2}^{\text {ext }} / S_{2}$. 
It is convenient to introduce a new set of asymmetry parameters which describe the difference in the slope of the energy $\kappa$ and the offset $a$ :

$$
\begin{aligned}
& h_{1}=h(1+\kappa / 2)+a / 2 \\
& h_{2}=h(1-\kappa / 2)-a / 2
\end{aligned}
$$

The parameter $h$ which describes the dependence on the magnetic field becomes

$$
h=I_{p} S B\left(1+\frac{\gamma \sigma}{4}\right)-\frac{1}{2} I_{p} \Phi_{0} \approx I_{p}\left(S B-\frac{1}{2} \Phi_{0}\right)
$$

For the approximation we assumed that the asymmetry is small, i.e. $\gamma, \sigma \ll 1$. For the parameter $\kappa$ describing the difference of the slopes we find

$$
\kappa=\frac{\gamma+\sigma}{1+\gamma \sigma / 4} \approx \gamma+\sigma
$$

This means that either a different persistent current $\gamma \neq 0$ or a different qubit area $\sigma \neq 0$ can lead to a different slope, but not a different magnetic field.

To a first order approximation the offset, $a$ depends only on the difference in applied magnetic field and a difference in qubit area $\sigma$

$$
\begin{aligned}
a & =B_{\text {asymm }} S I_{p}(1+\sigma / 2)(1+\gamma / 2)+I_{p} \Phi_{0} \frac{\sigma}{2} \frac{1-\gamma^{2} / 4}{1+\gamma \sigma / 4} \\
& \approx I_{p} S B_{\text {asymm }}+I_{p} \Phi_{0} \frac{\sigma}{2}
\end{aligned}
$$

The Hamiltonian now assumes the following form

$$
\mathcal{H}=\left(\begin{array}{cccc}
-2 h+j & t_{2} & t_{1} & 0 \\
t_{2} & -h \kappa-a-j & 0 & t_{1} \\
t_{1} & 0 & h \kappa+a-j & t_{2} \\
0 & t_{1} & t_{2} & 2 h+j
\end{array}\right)
$$

Figure 5.6 shows the energy levels for a general set of parameters. Far away from half a flux quantum in the qubit $\left(h \gg t_{1}, t_{2}, j,|a|\right)$ the eigenstates are the basic states of the two qubits.

$$
\begin{aligned}
& E_{0}=E_{|\downarrow \downarrow\rangle}=-2 h+j \\
& E_{1}=E_{|\downarrow \uparrow\rangle}=-h \kappa-a-j \\
& E_{2}=E_{|\uparrow \downarrow\rangle}=h \kappa+a-j \\
& E_{3}=E_{|\uparrow \uparrow\rangle}=2 h+j
\end{aligned}
$$


In spectroscopy one measures transitions between the ground state and the excited states [24]. The energy differences are

$$
\begin{aligned}
& \Delta_{|\downarrow \downarrow\rangle \rightarrow|\downarrow \uparrow\rangle}=(2-\kappa) h-a-2 j \\
& \Delta_{|\downarrow \downarrow\rangle \rightarrow|\uparrow \downarrow\rangle}=(2+\kappa) h+a-2 j \\
& \Delta_{|\downarrow \downarrow\rangle \rightarrow|\uparrow \uparrow\rangle}=4 h
\end{aligned}
$$

When the magnetic field is smaller than half a flux quantum $-h \gg t_{1}, t_{2}, j,|a|$, the eigenstates are

$$
\begin{aligned}
& E_{0}=E_{|\uparrow \uparrow\rangle}=-2(-h)+j \\
& E_{1}=E_{|\uparrow \downarrow\rangle}=-(-h) \kappa+a-j \\
& E_{2}=E_{|\downarrow \uparrow\rangle}=(-h) \kappa-a-j \\
& E_{3}=E_{|\downarrow \downarrow\rangle}=2(-h)+j
\end{aligned}
$$

and the energy differences are

$$
\begin{aligned}
& \Delta_{|\uparrow \uparrow\rangle \rightarrow|\uparrow \downarrow\rangle}=(2-\kappa)(-h)+a-2 j \\
& \Delta_{|\uparrow \uparrow\rangle \rightarrow|\downarrow \uparrow\rangle}=(2+\kappa)(-h)-a-2 j \\
& \Delta_{|\uparrow \uparrow\rangle \rightarrow|\uparrow \uparrow\rangle}=4(-h)
\end{aligned}
$$

However, the possibility to observe these transitions depends on the magnitude of the transition element. In spectroscopy it might also be possible to observe transitions between excited states.

Experimentally it is difficult to achieve a considerable degree of coupling. An attractive way to increase the coupling between the qubits is to introduce a large Josephson junction in the shared lead (see figure 5.7). The large junction acts as a Josephson inductance $L_{J}=\Phi_{0} /\left(2 \pi I_{0}\right)$, where $I_{0}$ is the critical current of this large junction. The Josephson inductance adds to the geometrical mutual inductance. However fabricating an additional large junction involves an extra fabrication step.

\subsubsection{Capacitive coupling}

Inductively coupling the qubits is not the only possibility. Figure 5.8 shows the coupling of two qubits with a big capacitor. This capacitor leads to a coupling of the form [25]

$$
\mathcal{H}_{\text {coupling }}=t\left(\sigma_{1}^{x} \sigma_{2}^{x}+\sigma_{1}^{y} \sigma_{2}^{y}\right)
$$




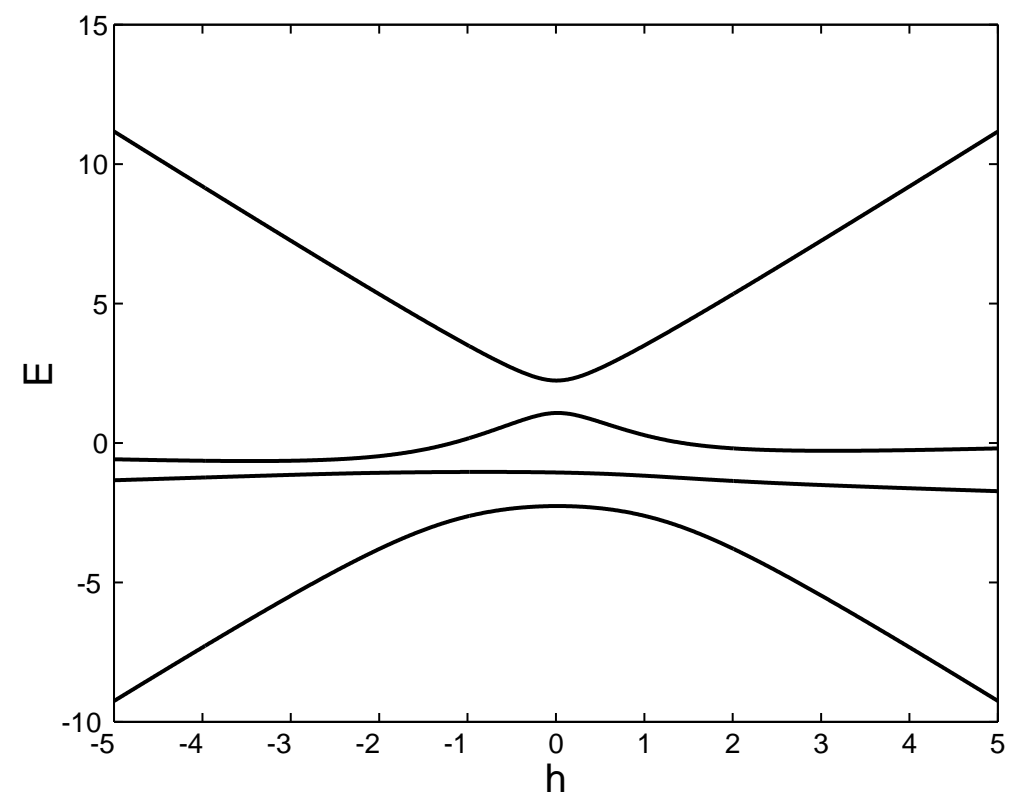

Figure 5.6: Energy levels of two coupled qubits for a general set of parameters. The parameters are $j=1$ for the coupling strength, $\kappa=0.1, a=0.2$ and $t_{1}=$ $1.2, t_{2}=0.8$.

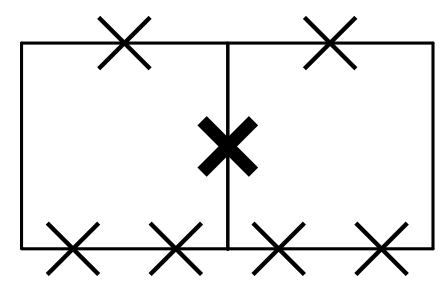

Figure 5.7: With an additional large Josephson junction in the shared lead the coupling between qubits is increased. 


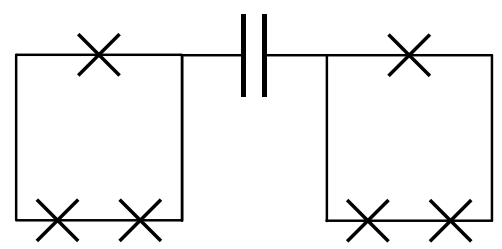

Figure 5.8: Coupling of the qubits with a capacitor leads to a coupling term $\sigma_{1}^{x} \sigma_{2}^{x}+\sigma_{1}^{y} \sigma_{2}^{y}$.

\subsection{Sample layout and design}

In the experiment one measures the states of the two qubits, both DC (for the ground state) and via microwave spectroscopy (for the excited state). This is done by measuring the flux produced by the circulating currents with a DC-SQUID. The two qubits are placed inside the SQUID. With a microwave antenna a small rf-field can be applied to the two qubits, which induces transitions between the states. With a wire placed near one of the two qubits, one can apply a small asymmetric static field.

The sample involves three fabrication steps: In the first step platinum is deposited. Resistors and markers for the following layer are made with this platinum layer. In the second step a layer of aluminum is deposited, which is oxidized with an oxygen plasma. This layer forms the bottom plate of a capacitor. In the last step the Josephson junctions are evaporated using shadow evaporation.

Figure 5.9 shows the circuit which was fabricated on a micro-chip [26]. The DC-SQUID is shunted with a capacitor and a resistor combination. This provides a good trade-off between low dissipation and a narrow switching histogram[27]. Furthermore all leads include a resistor to decouple the circuit from the measurement environment. Figure 5.10 shows a SEM (scanning electron image) of the whole circuit, Figure 5.11 the two qubits and the measuring SQUID. From the normal state resistance of the large qubit junctions 


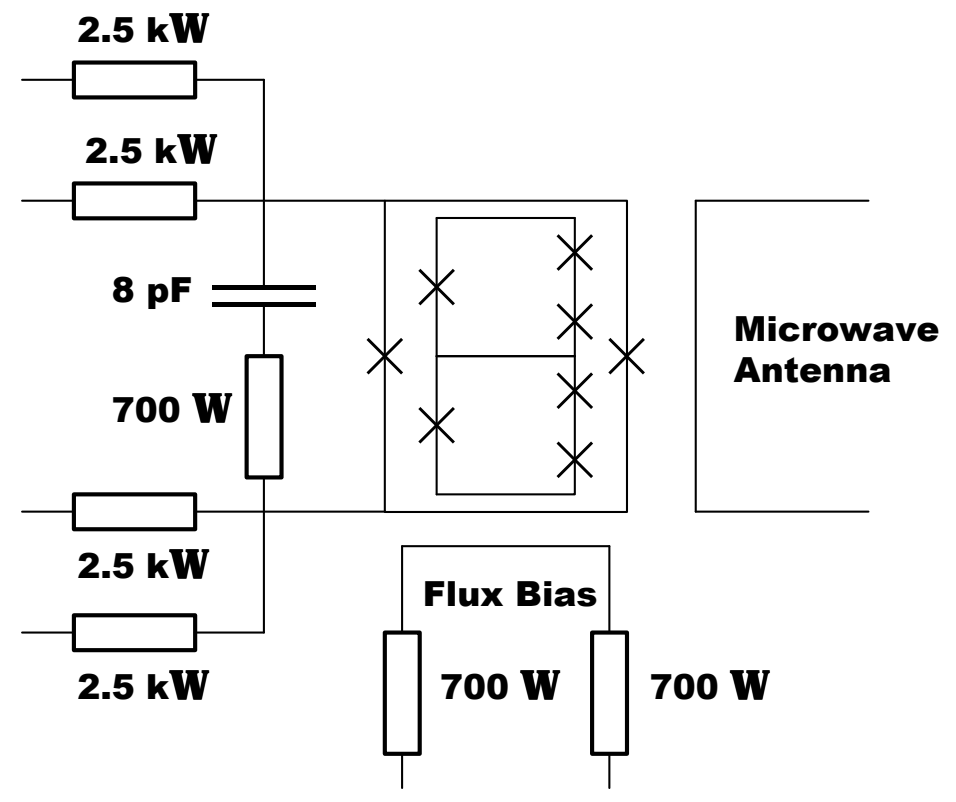

Figure 5.9: Scheme of the circuit fabricated on a micro-chip. The two qubits are surrounded by a DC-SQUID, which measures the flux produced by the two qubits. With the microwave antenna transitions in the qubit can be induced. The flux bias wire allows to apply an asymmetric magnetic field to the two qubits.

we calculate a critical current of $530 \mathrm{nA}$. With the area of the junctions we estimate the junction capacitance of $5 \mathrm{fF}$. With these values a ratio $E_{J} / E_{c}=65$ follows. The smaller junction of the qubit is designed to be 0.8 times the size of the two larger junctions in the qubit. Furthermore we compute the persistent current to be $350 \mathrm{nA}$ and the tunnel element $t=0.4 \mathrm{GHz}[13,23]$. In previous measurements flux noise has shown to be a severe source of noise and instability. This flux noise could be due to moving vortices trapped in superconducting plates near the qubit. To prevent these vortices from moving, holes are made in larger aluminum plates. Figure 5.12 shows a part of the shunt capacitor and the contact between aluminum structure and Pt shunt resistor. 


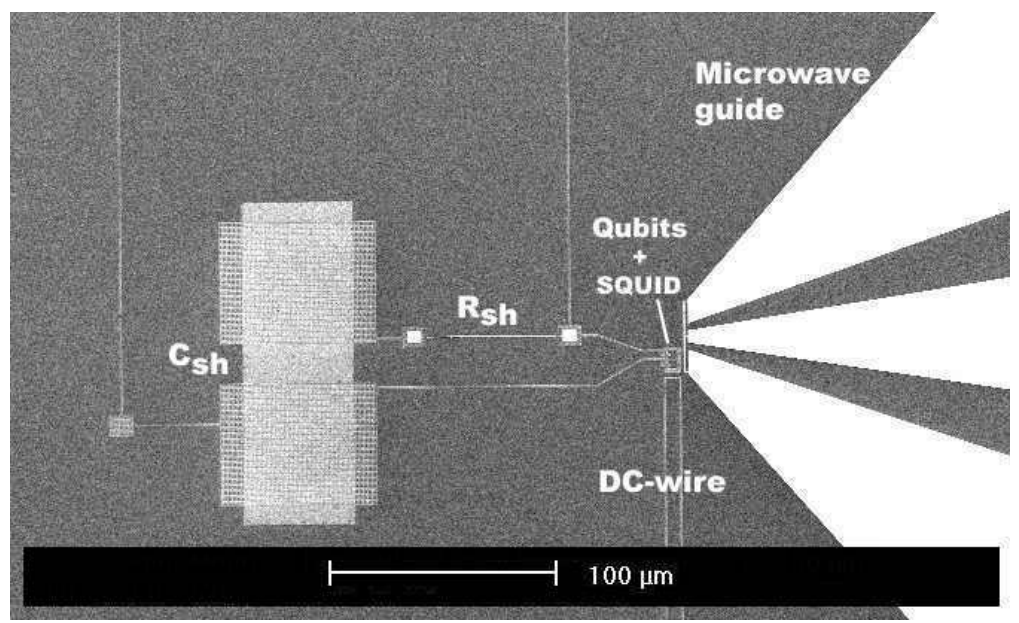

Figure 5.10: Scanning electron image (SEM) of the micro-chip. Clearly visible on the right is the microwave guide. On the left is the shunt capacitor $\mathrm{C}_{\mathrm{sh}}$ and the shunt resistor $\mathrm{R}_{\mathrm{sh}}$. The DC-wire for the flux bias extends to the bottom of the image.

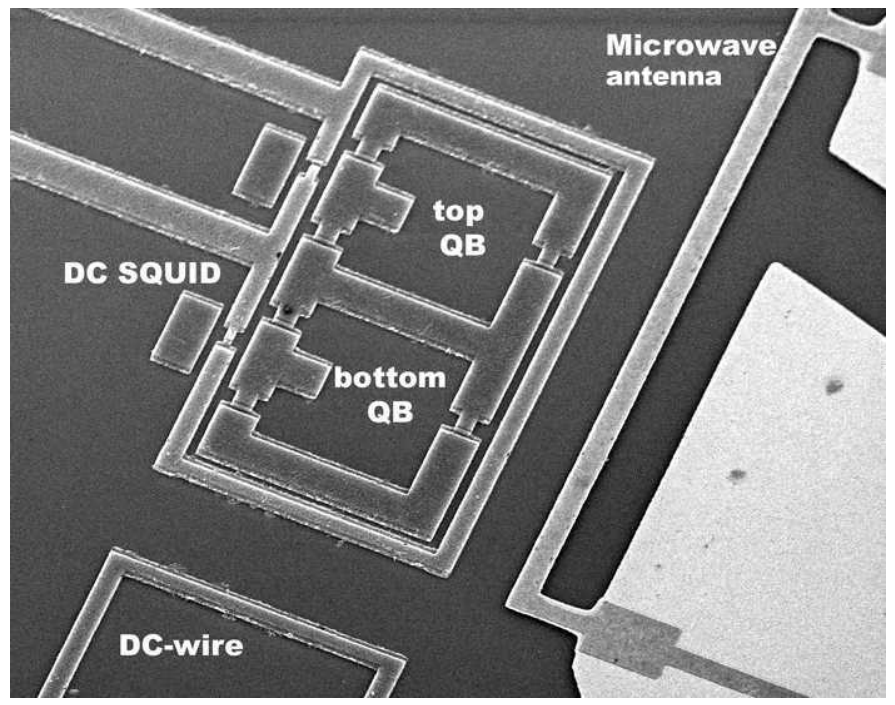

Figure 5.11: SEM image of the two coupled qubits surrounded by the DCSQUID. A part of the microwave antenna is visible on the right, which couples the microwave field to the qubits. Below the DC-wire is visible, which allows to apply an asymmetric magnetic field. 


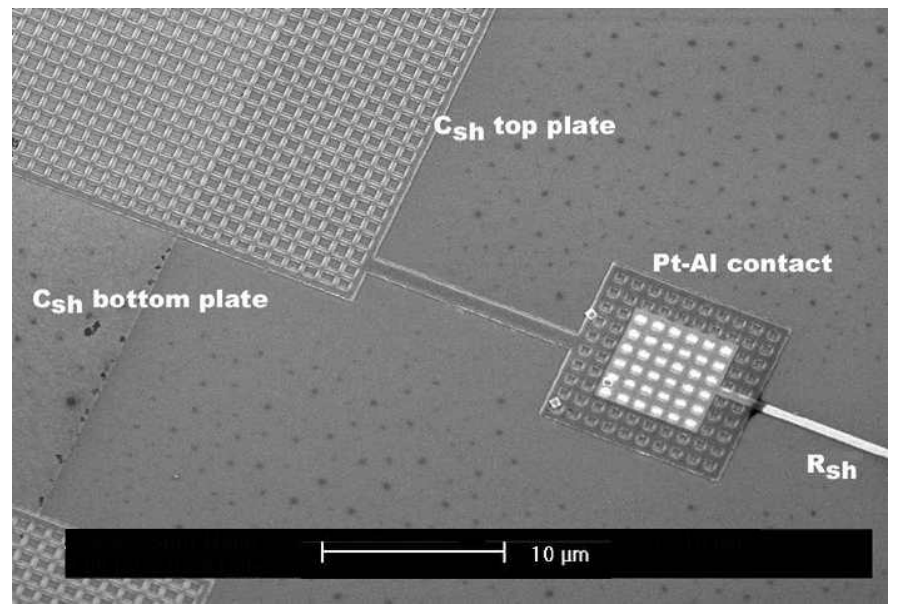

Figure 5.12: SEM image of a part of the shunt capacitor and the aluminum shunt resistor contact. The holes in the aluminum structure trap vortices and prevent them from moving around. 


\subsection{Measurements}

Measurements were performed in a dilution refrigerator at a base temperature of $20 \mathrm{mK}$. To suppress non-equilibrium noise, Pi-filters were used at room temperature and copper powder filters at sample temperature for the DC wires. The microwave wire was thermally anchored at $1 \mathrm{~K}$ and checked to be reflectionless till $25 \mathrm{GHz}$. The sample was placed in a cavity which provided a microwave environment without resonances. A magnetic field was applied with a large coil placed outside of the sample chamber. External magnetic fields were shielded by a double $\mu$-metal shield. We measured the switching current of the DC-SQUID. This was done by ramping the current in the SQUID and recording the current, when the SQUID switched to a voltage state. We repeated this procedure with a frequency of $200 \mathrm{~Hz}$ and average the switching current of typically 1000 measurements. Figure 5.13 shows the

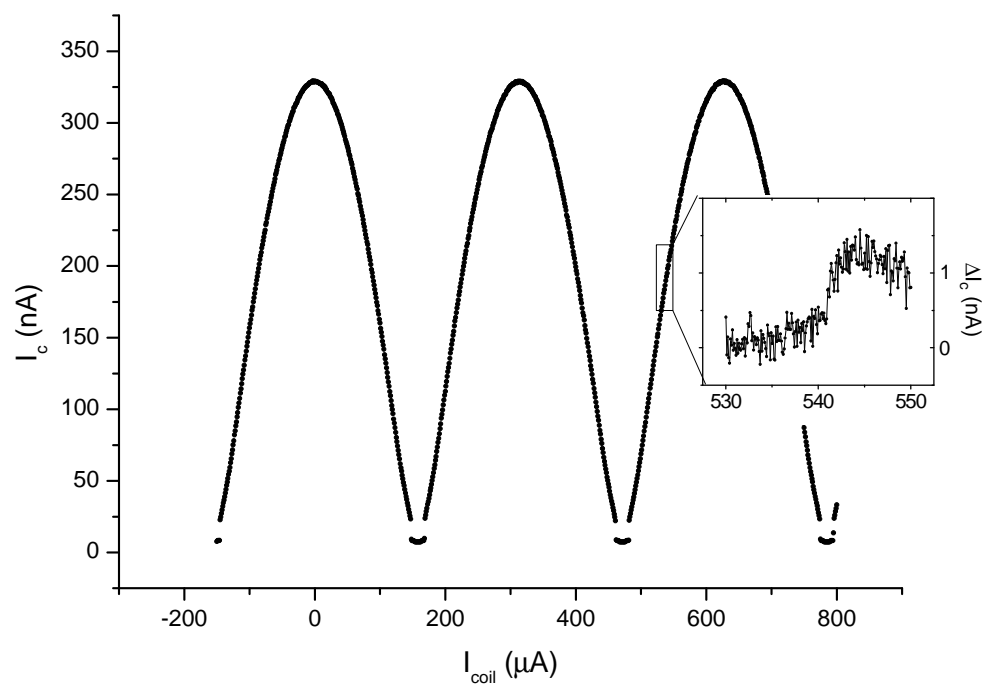

Figure 5.13: Switching current as a function of the applied magnetic field. The inset shows a a step which is due to the double qubits changing magnetization. The linear SQUID slope is subtracted to obtain the qubit signal only.

switching current of the SQUID as a function of the applied magnetic field. The switching current shows clear SQUID oscillations. At 1.7 flux quanta in the SQUID a small step in the slope is visible (inset figure 5.13 ). This field 
corresponds to $1 / 2$ flux quanta in the qubit. To obtain only the signal of the two qubits the linear SQUID slope was subtracted from the signal. The fact that we see only one step shows that the asymmetry between qubit areas $\sigma$ is small compared to the step width defined by the coupling parameter $j$. Otherwise we would observe two steps from the individual qubits.

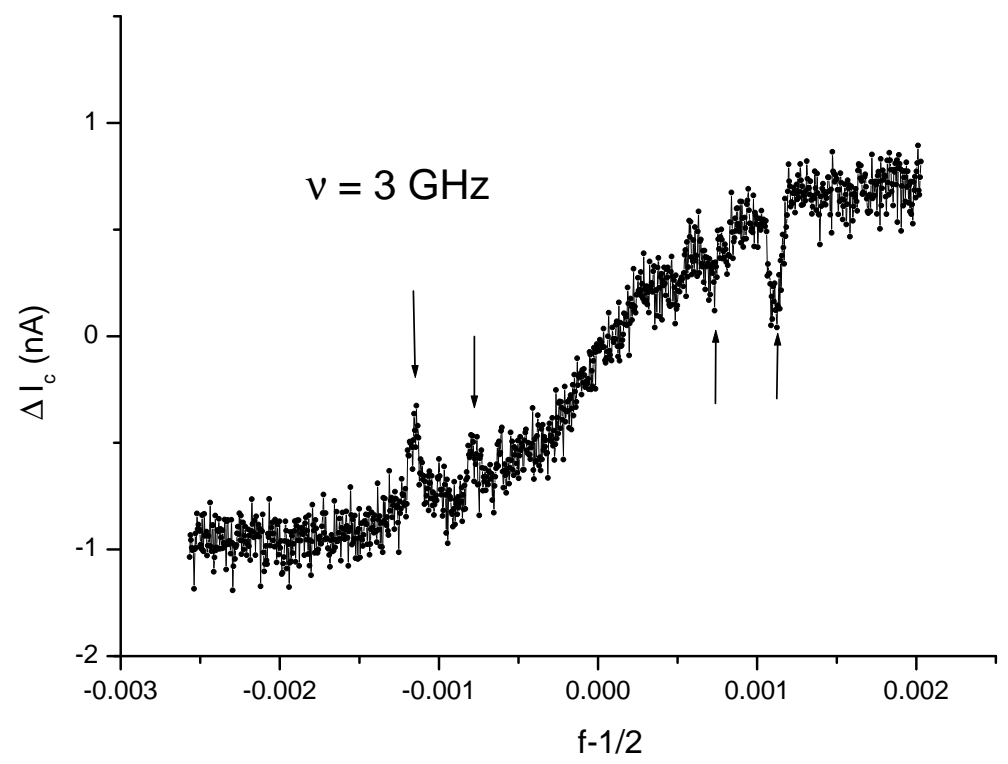

Figure 5.14: Double qubit step with a microwave frequency of $3 \mathrm{GHz}$ applied. Two clear resonance peaks on each side of the step are visible.

We applied a microwave signal to the qubits to induce transition between the qubit states. Figure 5.14 shows the step signal with a microwave of $3 \mathrm{GHz}$ applied. The microwave induced two peaks to the left of the step and two dips to the right. At these positions, the difference between energy levels matches the energy of the microwaves, so that the microwaves induces transitions [24]. If the system is measured repeatedly, the signal is the average of the magnetization of the two levels. Therefore the microwaves induce peaks at the lower part of the step and dips at the higher.

Instead of sweeping the magnetic field one can change the frequency of the microwave at a fixed field. Figure 5.15 shows the SQUID signal as a function of applied microwave frequency. Three broad resonances at 8, 14 
and $18 \mathrm{GHz}$ are observed. The position of these resonances does not change with the magnetic field. Therefore these resonances are not due to the qubit. Furthermore none of the resonances seems harmonically related. The plasma resonance of the SQUID together with the shunt capacitor is $1.8 \mathrm{GHz}$ and has a very small quality factor. It can therefore not account for these resonances. So far we have not found an explanation for these resonances. However, below $5 \mathrm{GHz}$ the signal is very flat. Figure 5.16 shows a measurement in the frequency range up to $5 \mathrm{GHz}$. Two dips can be observed. These two dips are qubit resonances and correspond to the dips observed in figure 5.14.

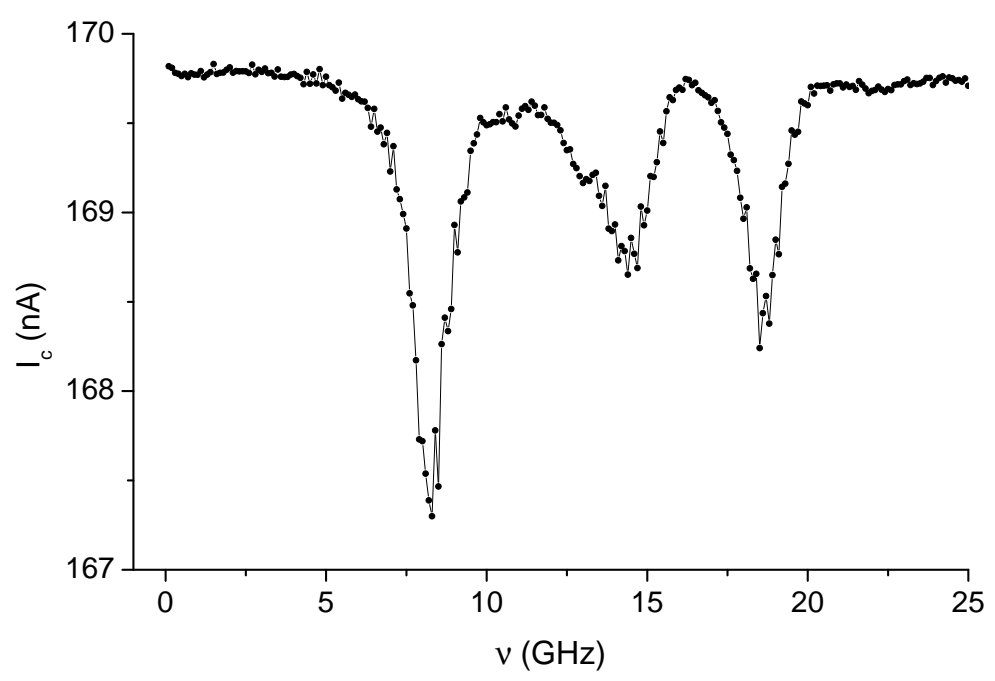

Figure 5.15: SQUID signal as a function of the microwave frequency at fixed magnetic field. The SQUID shows microwave resonances at 8, 14 and $18 \mathrm{GHz}$. These resonances are not due to the qubit as they do not shift with the applied magnetic field. However, below $5 \mathrm{GHz}$ the signal is very flat.

In order to confirm that these dips and peaks of figure 5.14 and 5.16 are due to the coupled qubits, the dependence of the flux position on the microwave frequency has to be studied. Figure 5.17 shows the peak or dip position (difference from half flux quantum) versus microwave frequency. One observes that far away from half a flux quantum the dips and peaks follow straight lines. At these high fields the states of coupled qubits are the basic states of the two qubits (See Eq. 5.19). The resonances correspond to transi- 


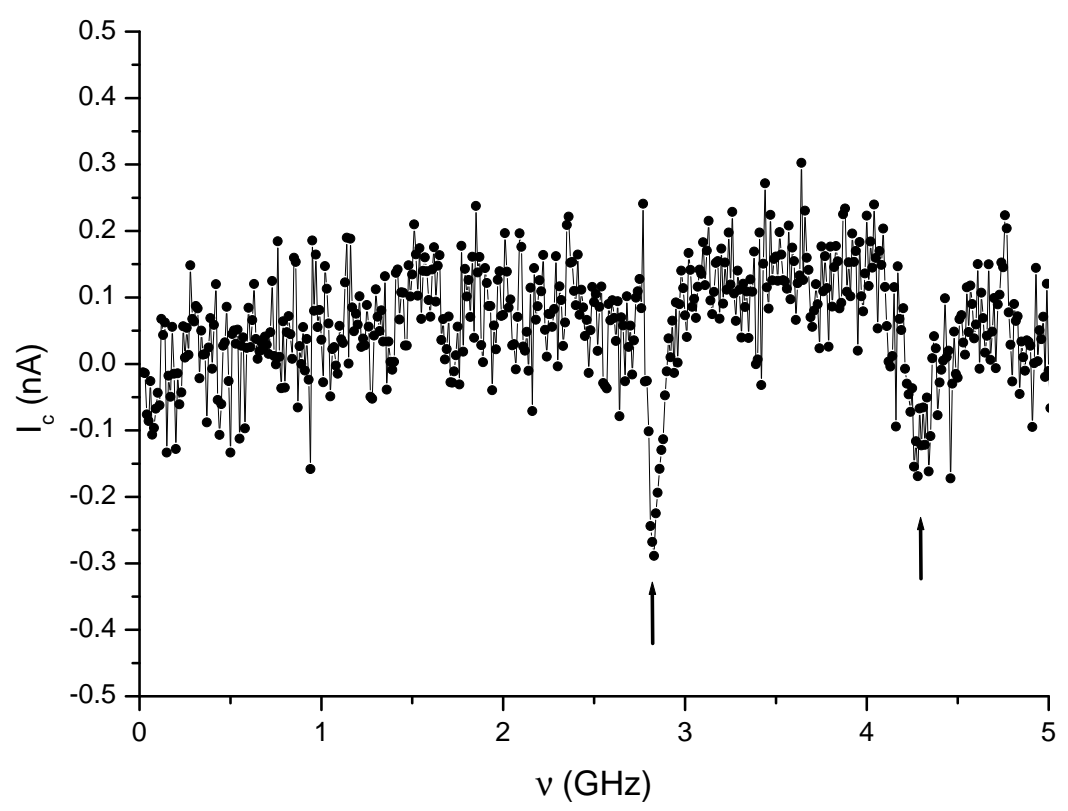

Figure 5.16: SQUID signal as a function of the microwave frequency below $5 \mathrm{GHz}$. Two qubit resonances are observable at $2.8 \mathrm{GHz}$ and $4.3 \mathrm{GHz}$. The magnetic field is $1.5 \mathrm{~m} \Phi_{0}$.

tion between these states where one of the qubits is flipped: $|\downarrow \downarrow\rangle \rightarrow|\downarrow \uparrow\rangle$ and $|\downarrow \downarrow\rangle \rightarrow|\uparrow \downarrow\rangle$. The transition energies are given by equation (5.20). Consistent with theoretical description is that the lines have the same slope for both sides. The slopes are $2.08 \mathrm{GHz} / \mathrm{m} \Phi_{0}$ and $2.89 \mathrm{GHz} / \mathrm{m} \Phi_{0}$. The corresponding persistent currents are $330 \mathrm{nA}$ and $460 \mathrm{nA}$. The lines cross on both sides. This behavior cannot be explained by the simple energy level considerations. As one can see from equations (5.19),(5.21) and figure 5.1, the energy levels of the states $|\uparrow \downarrow\rangle$ and $|\downarrow \uparrow\rangle$ cross only at one point.

In figure 5.17 also the peaks and dips of the transitions where both qubits are flipped $(|\downarrow \downarrow\rangle \rightarrow|\uparrow \uparrow\rangle)$ are missing. This could be due to the smaller transition element for this transition. One expects that a higher microwave power would induce this transition.

At magnetic fields near half a flux quantum, the position of the peaks start to deviate from the straight lines. At these fields the eigenstates of the 


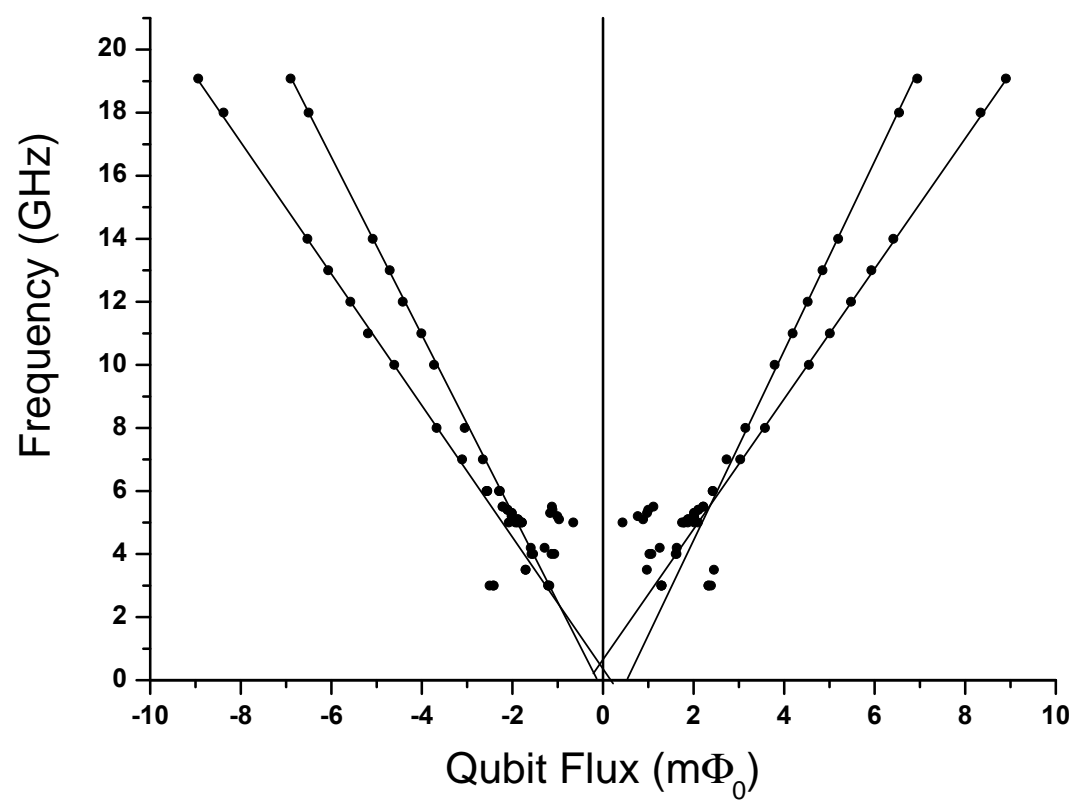

Figure 5.17: Peak and dips position versus microwave frequency. The x-axis labels the difference from half a flux quantum applied to the qubit. The straight lines are fits to the data at higher flux. They correspond to transitions where one qubit is flipped.

system are superposition of basic states. Therefore one expects a rounding of the peaks and dips positions. Due to the superposition of the states the difference in magnetization between the eigenstates decreases. Therefore the signal gets lower. As can be seen in figure 5.17, further measurements are needed to identify the transitions.

\subsection{Conclusions}

We study the coupling of persistent-current qubits. One practical way of coupling the qubits is to use the inductive interaction between the qubits. This interaction leads to a coupling term of the form $\sigma_{1}^{z} \sigma_{2}^{z}$. We study two perfectly identical qubits. The first excited state for this system is the singlet state and its energy is independent of the magnetic field. All the transmission 
elements including that state have zero amplitude, which means that this state is completely inaccessible. However two realistic qubits can never be perfectly identical. Therefore we treat the case of two coupled asymmetric qubits. We calculate the energy spectrum. Far away from half a flux quantum applied, the eigenstates are the basic states of the two qubits. The energy difference between the states have a linear dependence on the magnetic field. Near half a flux quantum applied, a quantum rounding occurs.

We have successfully fabricated a sample with two coupled qubits to perform spectroscopy. A SQUID with specially designed electromagnetic environment measured the two qubits. We have taken special care to prevent noise and instability caused by moving vortices.

Ground state measurements show a single step of the qubit magnetization. This shows that the qubit asymmetry is small compared with the coupling parameter. With microwaves applied we observe extra dips and peaks. These dips and peaks correspond to transitions between double qubit states. Far away from half a flux quantum applied to the qubits the dips follow straight lines. They correspond to transitions where one of the qubit is flipped, e.g. $|\downarrow \downarrow\rangle \rightarrow|\uparrow \uparrow\rangle$. As expected each line for the dips has a corresponding line for the peaks with the same negative slope. However, that the lines cross on both sides is inconsistent with the two qubit model. Further measurements are needed to reveal the structure in the region around half a flux quantum applied to the qubits.

We thank T. P. Orlando, Y. Nakamura, P. Hadley and A. Katan and for discussions and A. Lupascu and R. Schouten for technical assistance.

\section{References}

[1] D. P. DiVincenzo, "Quantum Computation," Science, 270255 (1995).

[2] S. Lloyd, Science, 2611569 (1993).

[3] S. Lloyd, Science, 263695 (1994).

[4] P. W. Shor, "Algorithms for quantum computation: discrete logarithms and factoring." in Proceedings, $35^{\text {th }}$ Annual Symposium on Foundations of Computer Science, 124, IEEE Press, Los Alamitos, CA 1994. 
[5] L. K. Grover, "Quantum mechanics helps in searching of a needle in a haystack," Phys. Rev. Lett. 79, 325, (1997).

[6] C. J. Turchette, C. J. Hood, W. Langem H. Mabuchi, H. J. Kimble, "Measurement of Conditional Phase Shifts for Quantum Logic," Phys. Rev. Lett. 75, 4710 (1995).

[7] C. Monroe, D. M. Meekhof, B. E. King, W. M. Itano, D. J. Wineland, "Demonstration of a Fundamental Quantum Logic Gate," Phys. Rev. Lett. 75, 4714 (1995).

[8] N. A. Gershenfeld and I. L. Chuang, "Bulk Spin-Resonance Quantum Computation," Science 275350 (1997).

[9] L. M. K. Vandersypen, M. Steffen, G. Breyta, C. S. Yannoni, M. H. Sherwood, I. L. Chuang, "Experimental realization of Shor's quantum factoring algorithm using nuclear magnetic resonance," Nature 414, 883 (2001).

[10] B. Kane, "A silicon-based nuclear spin quantum computer," Nature 393, 133 (1998).

[11] D. Loss, and D. DiVincenzo, "Quantum computation with quantum dots," Phys. Rev. A. 57, 120 (1998).

[12] J. E. Mooij, T. P. Orlando, L. Tian. C. H. van der Wal, L. S. Levitov, and J. J. Mazo, "A Superconducting Persistant Current Qubit," Science 285 1036, (1999).

[13] T. P. Orlando, J. E. Mooij, L. Tian, C. H. van der Wal, L. S. Levitov, S. Lloyd and J. J. Mazo, "Superconducting Persistent Current Qubit," Phys. Rev. B 60, 15398 (1999).

[14] Y. Makhlin, G. Schön, and A. Shnirman, "Josephson-junction qubits with controlled couplings," Nature 398, 305 (1999).

[15] Y. Makhlin, G. Schön and A. Shnirman, "Quantum-state engineering with Josephson-junction devices," Rev. Mod. Phys. 73, 357, (2001).

[16] Y. Nakamura, Yu. A. Pashkin, and J. S. Tsai, "Coherent control of macroscopic quantum states in a single-Cooper-pair box," Nature 398, 786 (1999). 
[17] C. H. van der Wal, A. C. J. ter Haar, F. K. Wilhelm, R. N. Schouten, C. J. P. M. Harmans, T. P. Orlando, S. Lloyd and J. E. Mooij, "Quantum Superposition of Macroscopic Persitent-Current States," Science 290, 773 (2000).

[18] J. R. Friedman, V. Patel, W. Chen, S. K. Tolpygo and J. E. Lukens, "Quantum Superpositions of Distinct Macroscopic States," Nature 406, 43, (2000).

[19] Y. Yu, S. Han, X. Chu, S. Chu, and Z. Wang, "Coherent Temporal Oscillations of Macroscopic Quantum States in a Josephson Junction," Science 296, 889 (2002).

[20] I. Chiorescu, Y. Nakamura, unpublished.

[21] D. Vion, A. Aassime, A. Cottet, P. Joyez, H. Pothier, C. Urbina, D. Esteve, and M. H. Devoret, "Manipulating the Quantum State of an Electrical Circuit," Science, 296, 886 (2002).

[22] W. J. Elion, M. Matters, U. Geigenmüller and J. E. Mooij, Nature 371, 594 (1994).

[23] http://qt.tn.tudelft.nl/〜majer/qubit

[24] C. Cohen-Tannoudji, B. Diu, F. Laloë, "Quantum Mechanics," Chapter IV, John Wiley \& Sons, New York (1977).

[25] L. S. Levitov, T. P. Orlando, J. B. Majer, and J. E. Mooij, "Quantum spin chains and Majorana states in arrays of coupled qubits," condmat/0108266.

[26] F. Paauw, "Inductively coupling two Josephson persistent current qubits," Intermediate Report, TU Delft, June 2002.

[27] A. Katan, "Switching behavior of dc-SQUIDs with frequency-dependent damping: measurement tools for the Josephson persitent-current qubit," Final Report, TU Delft, May 2002. 


\section{Summary}

\section{Superconducting Quantum Circuits}

This thesis describes a number of experiments with superconducting circuits containing small Josephson junctions. The circuits are made out of aluminum islands which are interconnected with a very thin insulating aluminum oxide layer. The connections form a Josephson junction. The current trough the junction is related to the superconducting phase difference across the junction and described by the well-known Josephson relation. The characteristic energy scale for this effect is the Josephson energy, which is a measure for the strength of the coupling of the islands. Due to the parallel plate geometry of the junction a capacitance is formed. This capacitance leads to a charging effect. The characteristic energy scale for this effect is given by the charging energy. With the shadow evaporation technique it is possible to make the Josephson junction area very small and the two characteristic energy scales of the same order of magnitude. Charge and phase are conjugate variables. Therefore a circuit containing such small Josephson junctions exhibits quantum effects. The microfabrication technique used in this thesis allows fabrication of solid circuits with controlled quantum mechanical behavior.

The first two chapters (chapters 2 and 3 ) describe experiments with quasi one-dimensional Josephson junction arrays. The array consists of a long, narrow network of Josephson junctions arranged in a rectangular lattice. Applying a magnetic field perpendicular to the array induces vortices in the array. The superconducting electrodes along the length of the array repel the vortices and force them to move in one dimension. These vortices behave like particles. The potential of these particles is defined by the Josephson 
junction array and is proportional to the Josephson energy of the junctions. The mass of the vortices is proportional to the capacitance of the junctions. Therefore it is possible to make a particle in weak potential with a low mass which behaves like a quantum particle. It is possible to accurately control the density of the vortices, apply a force and measure the speed. Furthermore the potential for the vortices can be influenced by changing the strength and the position of the junctions.

Chapter 2 describes an experiment where the vortices move in an asymmetric, periodic potential, a so-called ratchet potential. Only the strongly asymmetric sample exhibits a ratchet effect, a difference of vortex speed between forward and backward driving. The missing ratchet effect in the weakly asymmetric sample can be explained by the band structure. This sample only has one energy band, which is relevant for the transport. Because a single energy band is always symmetric, a ratchet effect is not possible for this sample. The voltage-current curves of all the four samples follow a perfect power-law, with a power higher than one. This confirms the quantum nature of the vortex transport. It is observed that additional friction increases the ratchet effect. Chapter 2 also provides a new theory, which describes the quantum transport with only few bands.

Vortices in a quasi one-dimensional Josephson junctions array have a very large interaction and form a rigid chain. In chapter 3 it is shown that one can control the number of vortices in the chain one by one down to only 6 vortices in an array of length 303. The second part of this chapter describes an attempt to lower the interaction between the vortices. This can be done by replacing the superconducting electrodes with Josephson junctions, but the confining potential is lowered as well. Measurements show the confining potential is weak and that the vortices escape via the edge already at low driving force.

Often superconducting circuits require an imposed phase difference. Usually this phase difference is applied with a magnetic field or with $\pi$-junctions. Chapter 4 demonstrates a surprisingly simple phase-bias tool, based on trapped fluxoids in a superconducting ring. The phase bias has been realized in two superconducting quantum interference devices (SQUID), where the critical current versus magnetic field is shown to be shifted by $\pi / 2$ and $\pi$. Several 
applications of this phase-bias tool are proposed: a persistent-current qubit and a SQUID, which make use of a gradiometer design to reduce the flux noise. Also a new class of superconducting logics is proposed which does not require resistive elements.

The last chapter treats the coupling of two persistent-current qubits. The qubits are coupled inductively by placing them next to each other which leads to a Ising kind of coupling. The energy spectrum for two qubits with such a coupling is calculated for the case of two identical as well as for two non identical qubits. The signal of the two qubits is measured with a SQUID that surrounds the two qubits. With microwaves it is possible two induce transitions between the two qubit energy states. Preliminary measurements show transitions where one qubit state is flipped.

It can be concluded that circuits based on Josephson junctions can provide a large range of applications: starting from studying fundamental quantum effects like the ratchet effect in chapter 2 and the coupling of solid state quantum bits in chapter 5 to new superconducting logics as proposed in chapter 4.

Johannes B. Majer

October 2002 


\section{Samenvatting}

\section{Supergeleidende Quantum Circuits}

Dit proefschrift beschrijft een aantal experimenten met supergeleidende circuits die kleine Josephson-juncties bevatten. De circuits zijn gefabriceerd van aluminium eilandjes die met extreem dunne isolerende lagen van aluminiumoxide aan elkaar verbonden zijn. Deze connecties vormen Josephsonjuncties. De stroom door deze juncties is gerelateerd aan het supergeleidende faseverschil over de juncties en wordt beschreven door de bekende Josephsonrelatie. De karakteristieke energieschaal voor dit effect is de Josephsonenergie, welke een maat is voor de sterkte van de koppeling tussen de eilanden. Door de parallelle-plaatgeometrie van de juncties ontstaat een capaciteit tussen de eilanden. Deze capaciteit leidt tot een ladingseffect tussen de eilanden met de ladingsenergie als karakteristieke energieschaal. Met schaduwopdamptechniek kan de oppervlakte van de Josephson-juncties heel erg klein gemaakt worden en kunnen de twee karakteristieke energieën van de zelfde orde van grootte gemaakt worden. De fase en lading van een Josephsonjunctie zijn geconjugeerde variabelen. Daardoor vertoont een circuit met Josephson-juncties quantummechanische effecten. Met de microfabricagetechnieken die voor dit onderzoek gebruikt zijn, kunnen vaste-stofcircuits met gecontroleerd quantumgedrag gemaakt worden.

In de eerste twee hoofdstukken (hoofdstukken 2 en 3) worden experimenten met quasi ééndimensionale Josephson-junctie roosters beschreven. De roosters bestaan uit een lang en smal netwerk van Josephson-juncties gerangschikt in een rechthoekig rooster. Door het aanleggen van een magneetveld worden vortices geïnduceerd. De supergeleidende electroden langs 
de kant van het rooster stoten de vortices af en dwingen ze in één dimensie te bewegen. De vortices gedragen zich als deeltjes. De potentiaal van deze deeltjes wordt gedefinieerd door het onderliggende rooster en is recht evenredig met de Josephson-energie van de juncties. De massa van de vortices is proportioneel met de capaciteit van de juncties. Daardoor is het mogelijk een deeltje in een zwakke potentiaal met een geringe massa te maken dat zich quantummechanisch gedraagt. Het is ook mogelijk de dichtheid van de deeltjes nauwkeurig te controleren, een kracht uit te oefenen en de snelheid te meten. Verder kan de potentiaal van de vortices veranderd worden door de sterkte en de positie van de juncties.

Hoofdstuk 2 beschrijft een experiment waar de vortices in een asymmetrische, periodieke potentiaal bewegen, een zogeheten ratelpotentiaal. Van de vier samples laat slechts het sterk asymmetrische sample een rateleffect (een verschil van snelheid van de vortices tussen vooruit en achteruit aandrijven) zien. Het missende rateleffect voor het zwak asymmetrische sample kan verklaard worden met de bandenstructuur. Dit sample heeft slechts één energieband die voor het transport relevant is. Omdat een enkele energieband altijd symmetrisch is, ontstaat er geen rateleffect voor dit sample. De spanning-stroom curven van alle vier samples volgen een perfecte machtswet, met een macht groter dan één. Dit feit bevestigt het quantumgedrag van de vortices. Het is waargenomen dat het rateleffect van het sterk asymmetrische sample door meer frictie toeneemt. Hoofdstuk 2 bevat ook een nieuwe theorie die de quantum transport met weinig energiebanden beschrijft.

Vortices in een quasi ééndimensionaal Josephson-junctie rooster voelen een sterke interactie en vormen een stijve ketting. In hoofdstuk 3 wordt aangetoond dat men het aantal vortices één voor één kan controleren tot slechts 6 vortices in een rooster met lengte 303. Het tweede deel van dit hoofdstuk beschrijft een poging de interactie tussen de vortices te reduceren. Dit kan gedaan worden door het vervangen van de supergeleidende elektroden met Josephson-juncties. Maar daarmee wordt ook de opsluitpotentiaal verlaagd. Metingen tonen dat de opsluitpotentiaal erg zwak is en dat de vortices al bij lage aandrijfkrachten door de randen ontsnappen.

Vaak vereisen supergeleidende circuits een aangelegd faseverschil. Gewoonlijk wordt dit door middel van een magnetisch veld of met $\pi$-juncties aan- 
gelegd. Hoofdstuk 4 beschrijft een verrassend eenvoudige methode gebaseerd op het opsluiten van een fluxoid in een supergeleidende ring. Het faseverschil is experimenteel aangetoond met twee supergeleidende quantum interferentie detectors (SQUID) waar het gedrag van de kritische stroom tegen het magneetveld over $\pi / 2$ of $\pi$ verschoven is. Meerdere toepassingen van deze methode worden voorgesteld: een persisterende stroom quantumbit en een SQUID, die met behulp van een gradiometer ontwerp minder gevoelig voor fluxruis zijn. Ook een nieuwe soort supergeleidende logica zonder weerstanden wordt voorgesteld.

Het laatste hoofdstuk behandelt het koppelen van twee persisterende stroom quantumbits. De quantumbits worden inductief gekoppeld door ze naast elkaar te leggen, wat leidt tot een soort van Ising interactie. Het energiespectrum van twee quantumbits met een dergelijke koppeling wordt berekend voor het geval van twee identieke en twee verschillende quantumbits. Het signaal van de twee quantumbits wordt met behulp van een omringende SQUID. Met behulp van microgolven kunnen overgangen tussen energietoestanden geïnduceerd worden. Voorlopige metingen tonen overgangen waar de toestand van een van de twee quantumbits omgedraaid wordt.

Men kan concluderen dat circuits met Josephson juncties een breed bereik aan toepassingen hebben: van het bestuderen van fundamentele quantum effecten zoals het quantumrateleffect in hoofdstuk 2 en het koppelen van vaste stof quantumbits tot de nieuwe supergeleidende elektronica die in hoofdstuk 5 voorgesteld wordt.

Johannes B. Majer

Delft, oktober 2002 


\section{Zusammenfassung}

\section{Supraleitende Quanten Stromkreise}

Die vorliegende Dissertation beschreibt einige Experimente mit supraleitenden Stromkreisen, die kleine Josephson Verbindungen enthalten. Die Stromkreise werden aus Aluminium-Inseln hergestellt, die durch sehr dünne isolierende Aluminiumoxid-Lagen verbunden sind. Diese Verbindungen sind Josephson Verbindung. Der Strom durch diese Verbindungen ist abhängig von der supraleitenden Phasendifferenz und wird beschrieben mit der bekannten Josephson-Gleichung. Die charakteristische Energie für diesen Effekt ist die Josephson-Energie, die die Stärke der Kopplung zwischen den Inseln angibt. Durch die Geometrie aus parallelen Platten der Verbindung entsteht eine Kapazität zwischen den Inseln. Diese Kapazität führt zu einem Ladungseffekt. Die charakteristische Energie dieses Effektes ist die Ladungsenergie. Durch die Schattenbedampfungstechnik können Josephson Verbundungen mit sehr kleinen Oberflächen hergestellt werden, und die zwei charakteristischen Energien weisen dieselbe Grössenordnung auf. Ladung und Phase sind konjugierte Variablen. Darum zeigt ein Stromkreis mit solchen Verbindungen quantenmechanische Effekte. Die Mikrofabrikationstechnologie, die für diese Arbeit verwendet wurde, erlaubt die Herstellung von Festkörperschaltungen mit kontrollierten quantenmechanischen Eigenschaften.

Die Kapitel 2 und 3 beschreiben Experimente mit quasi-eindimensionalen Josephson-Verbindung-Gittern. Diese bestehen aus langen und schmalen Netzwerken von rechtwinklig angeordneten Josephson Verbindungen. Mit dem Anlegen eines magnetischen Feldes werden im Gitter Vortices induziert. Die supraleitenden Elektroden entlang der Seite des Gitters stossen die Vortices ab und zwingen sie, sich in einer Dimension zu bewegen. Diese Vor- 
tices verhalten sich wie Teilchen. Ihr Potential wird durch das JosephsonVerbindung-Gitter definiert und ist proportional zur Josephson-Energie der Verbindungen. Die Masse der Teilchen ist proportional zur Kapazität der Verbindungen. Darum ist es möglich, ein Teilchen in einem schwachen Potential mit kleiner Masse zu erzeugen, das sich wie ein quantenmechanisches Teilchen verhält. Weiter ist möglich, die Dichte der Teilchen zu kontrollieren, eine Kraft auszuüben und die Geschwindigkeit zu messen. Zudem kann das Potential der Vortices beeinflusst werden durch das ändern von Grösse und Position der Verbindungen.

Kapitel 2 beschreibt ein Experiment, bei dem sich die Vortices in einem asymmetrischen periodischen Potential bewegen, einem so genannten RätschenPotential. Nur die stark asymmetrische Probe zeigt einen Rätschen-Effekt, eine Differenz der Geschwindigkeit zwischen Vorwärts- und Rückwärtstreiben. Der fehlende Rätschen-Effekt für die schwach asymmetrische Probe kann anhand der Bänderstruktur verstanden werden. Diese Probe weist nur ein Energieband auf, das relevant für den Transport ist. Weil ein einzelnes Energieband immer symmetrisch ist, kann eine solche Probe niemals einen Rätschen-Effekt zeigen. Die Strom-Spannungs-Kurven für alle vier Proben folgen einem perfekten Potenz-Gesetz, mit einem Exponenten grösser als eins. Dies bestätigt das quantenmechanische Verhalten des Vortex-Transports. Man beobachtet auch, dass der Rätschen-Effekt mit zunehmender Dissipation zunimmt. Zudem wird in Kapitel 2 eine neue Theorie präsentiert, die den quantenmechanischen Transport in einem System mit nur wenigen Energiebändern beschreiben kann.

Vortices in einem quasi-eindimensionalen Josephson-Verbindung-Gitter haben eine grossen Wechselwirkung und bilden eine steife Kette. Im Kapitel 3 wird gezeigt, dass auf diese Weise die Anzahl der Vortices einzeln kontrolliert werden kann bis herunter auf 6 Vortices, in einem Gitter mit der Länge 303. Der zweite Teil des Kapitels beschreibt einen Versuch, die Wechselwirkung zwischen den Vortices zu verringern. Dies kann durch das Ersetzen der supraleitenden Elektroden durch Josephson Junctions geschehen, aber dadurch wird auch das Beschränkungspotential verringert. Messungen zeigen, dass das Beschränkungspotential sehr schwach ist und die Vortices schon bei kleinen Kräften durch den Rand entkommen. 
Oft erfordern supraleitende Stromkreise eine fest angelegte Phasendifferenz. Normalerweise wird eine Phasendifferenz mit einem Magnetfeld oder mit $\pi$-Verbindungen angelegt. Kapitel 4 demonstriert eine überraschend einfache Methode, basierend auf dem Einfangen eines Fluxoids in einem supraleitenden Ring. Das Anlegen der Phasendifferenz wird mittels zweier supraleitender Quantum-Interferenz-Detektoren (SQUID) demonstriert, wo die kritische Strom-Magnetfeld-Abhängigkeit um $\pi / 2$ respektive $\pi$ verschoben ist. Mehrere Anwendungen dieser Methode werden vorgeschlagen: ein persistent-Strom-Quantenbit und ein SQUID, wobei mittels eines GradiometerDesigns das Flussrauschen reduziert wird. Auch wird eine neue Klasse supraleitender Logik vorgeschlagen, die keine Widerstände benötigt.

Das letzte Kapitel behandelt das Koppeln von persistent-Strom-Quantenbits. Die Quantenbits werden induktiv gekoppelt durch Nebeneinanderplazieren. Das führt zu einer Ising-artigen Kopplung. Es wird das Energiespektrum zweier Quantenbits berechnet für den Fall zweier identischer und auch zweier nicht identischer Quantenbits. Das Signal der zwei Quantenbits wird mit einem SQUID gemessen, der beide Quantenbits umringt. Mit Mikrowellenstrahlung können übergänge zwischen den zwei QuantenbitZuständen induziert werden. Vorläufige Messungen zeigen Übergänge, wobei ein Quantenbit seinen Zustand ändert.

Abschliessend kann festgestellt werden, dass Stromkreise, basierend auf Josephson Junctions, einen grossen Bereich von Anwendungen haben, beginnend mit dem Studium fundamentaler Quanteneffekte wie dem RätschenEffekt in Kapitel 2 und dem Koppeln von Festkörper-Quantenbits im Kapitel 5 , bis zu neuer supraleitender Logik, wie sie im Kapitel 4 vorgestellt wird.

Johannes B. Majer

Delft, Oktober 2002 


\section{List of publications}

1. Simple phase bias for superconducting circuits

J. B. Majer, J.R. Butcher, J.E. Mooij, Applied Physics Letters 80, 3638

2. Quantum Ratchets with Few Bands below the Barrier

M. Grifoni, M. S. Ferreira, J. Peguiron, and J. B. Majer Physical Review Letters 89, 146801

3. Quantum ratchet effect for vortices

J.B. Majer, J. Peguiron, M. Grifoni, M. Tusveld, J.E. Mooij submitted to Physical Review Letters

4. Quantum spin chains and Majorana states in arrays of coupled qubits

L. S. Levitov, T. P. Orlando, J. B. Majer, J. E. Mooij, cond-mat/0108266 


\title{
Curriculum Vitae
}

\author{
Johannes Balthasar Majer
}

1 April 1972 Born in Weinfelden, Switzerland

1987-1992 High School at the Kantonschule

in Kreuzlingen, Switzerland

January 1992 Matura Typus C (natural sciences)

1992-1997 Graduate Study Physics

at the Eidgenössische Technische Hochschule

in Zürich, Switzwerland

November 1997 Diploma in Physics, cum laude

Diploma thesis: "Grenzflächen- und Legierungsstreuung

in III-IV-Quantentöpfen"

under supervision of prof.dr. Klaus Ensslin

1998-2002 Ph.D research at Delft University of Technology

Subject: Superconducting Quantum Circuits

under supervision of prof.dr.ir. J. E. Mooij 
